Measurements of optically and chemically stimulated neurotransmitter release in Drosophila

Ning Xiao

Zibo, Shandong, China

B.S., University of Science and Technology of China, 2009

A Dissertation presented to the Graduate Faculty of the University of Virginia in Candidacy for the Degree of Doctor of Philosophy

Department of Chemistry

University of Virginia

July, 2014 


\section{Measurements of optically and chemically stimulated neurotransmitter release in Drosophila}

\section{Abstract}

Model systems are needed in the study of neurotransmission mechanisms and in the search for treatments for diseases of the central nervous system. Drosophila melanogaster, the fruit fly, is an attractive model organism because it has a complex nervous system that has many conserved pathways with mammals and it is easy to genetically alter. To use Drosophila as a model system to study the molecular and genetic basis for neurological diseases, a comprehensive understanding of its neurotransmitter systems is necessary.

My dissertation research focuses on measurements of real-time neurotransmitter changes in Drosophila central nervous system using fast-scan cyclic voltammetry at carbon-fiber microelectrodes. My research concentrates on developing methods in three aspects: the sensor for detection, the modeling method and the control of neurotransmitter release. Chapter 2 introduces a method to modify the microelectrode with aligned carbon nanotube forests. The aligned carbon nanotube forests greatly improved electrode sensitivity and selectivity, and facilitated faster measurement. Chapter 3 describes optogenetic control of serotonin or dopamine release with pulsed optical stimulations. A modeling method which has been well established in mammalian models was exploited to estimate dopamine and serotonin release and clearance kinetics in Drosophila larva. We found the $V_{\max }$ and $K_{\mathrm{m}}$ for serotonin and dopamine in Drosophila were similar to their values in mammals, but the amount of neurotransmitter released per pulse was smaller. Chapter 4 employs a chemical stimulation method to investigate the releasable and reserve dopamine pools in Drosophila larva. With ATP/P2X $\mathrm{X}_{2}$ mediated stimulation, we found both synthesis and reuptake were needed to 
maintain the releasable dopamine pool, with synthesis playing a major part in long-term replenishment and reuptake being more important for short-term replenishment and there was no cocaine-activated reserve pool of dopamine in Drosophila. These studies overcome critical technical barriers to get a better understanding of dopamine and serotonin regulation in Drosophila, and strengthen the use of this model organism for the study of mechanisms underlying human behaviors and neurodegenerative diseases. 


\section{Table of Contents}

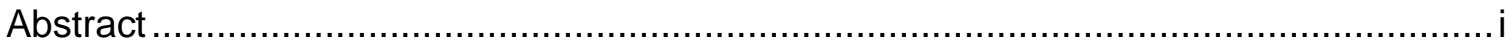

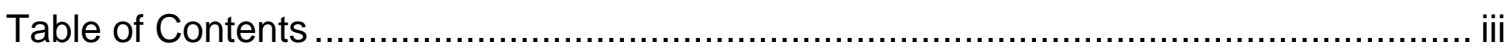

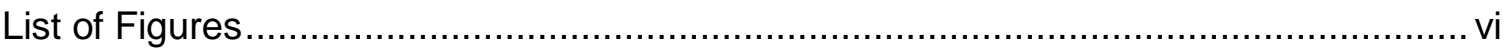

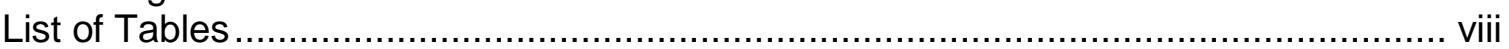

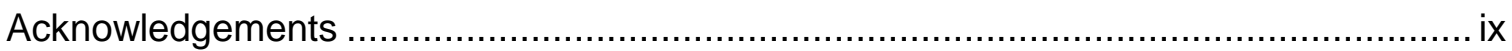

Chapter 1: Introduction ................................................................................ 1

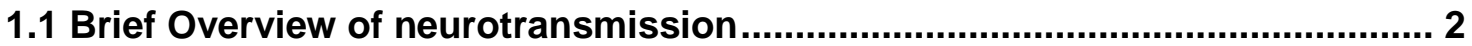

1.2 Electrochemical measurements of neurotransmitters in vivo......................... 5

1.2.1 Requirements for in vivo electrochemical sensor ................................... 5

1.2.2 Fast-scan cyclic voltammetry with carbon-fiber microelectrode...................... 7

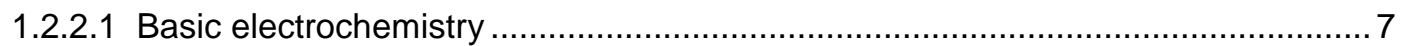

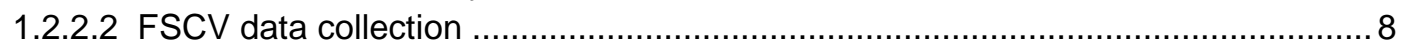

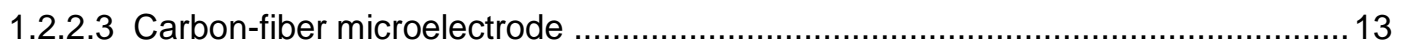

1.2.3 Microelectrode modifications …............................................................ 16

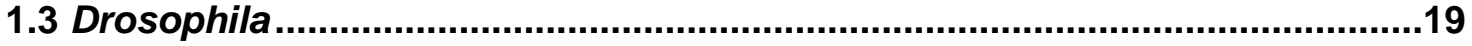

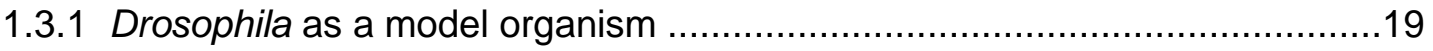

1.3.2 Neurotransmitter systems in Drosophila ............................................21

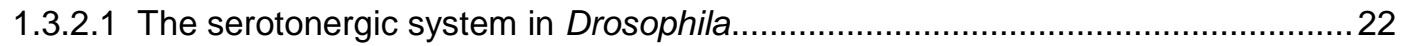

1.3.3 Tools for stimulating neurotransmitter release in Drosophila.......................24

1.3.4 Measurements of neurotransmission in Drosophila with FSCV ....................27

1.4 Reference List ......................................................................................28

Chapter 2: Rapid, sensitive detection of neurotransmitters at microelectrodes modified with self-assembled SWCNT forests ..................34

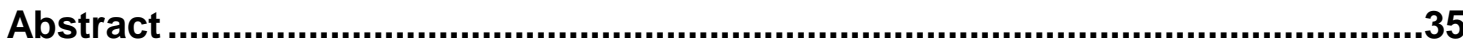

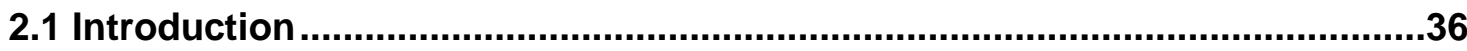

2.2 Results and Discussion.....................................................................................38

2.2.1 Fast-scan cyclic voltammetry of dopamine at SWCNT forest electrodes.......38

2.2.2 Effects of different coating methods ........................................................42

2.2.3 Enhanced electrochemical detection for other neurochemicals.....................45

2.2.4 Improved temporal resolution at highly sensitive microelectrodes..................46

2.2.5 Measurements in Drosophila ............................................................. 48

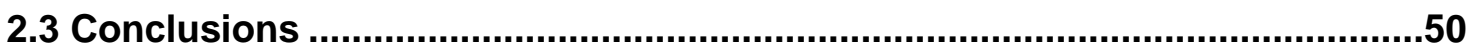

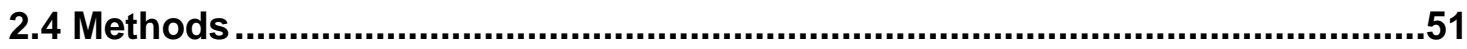

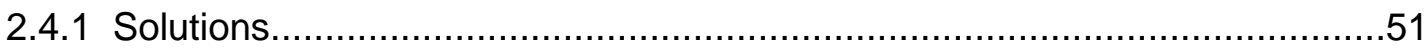

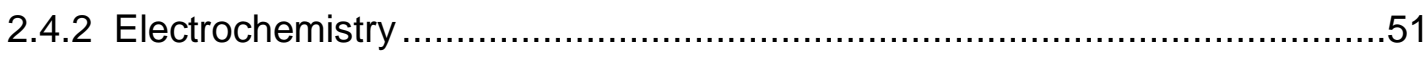

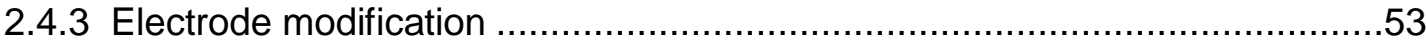

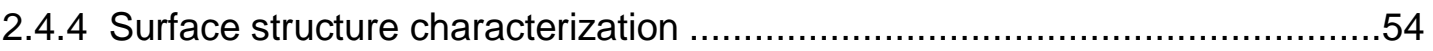

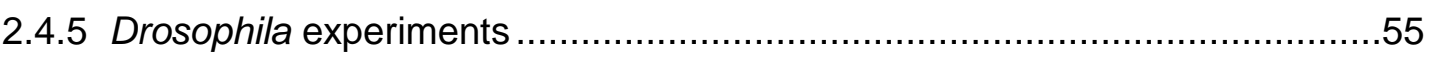

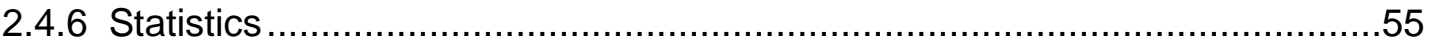

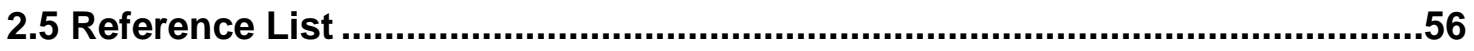




\section{Chapter 3: Optogenetic control of serotonin and dopamine release in}

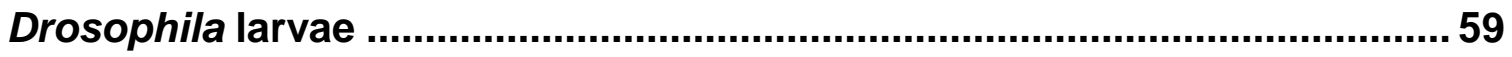

Abstract

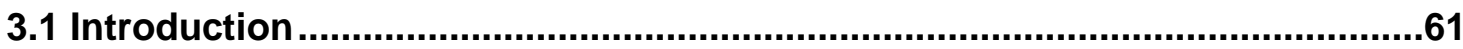

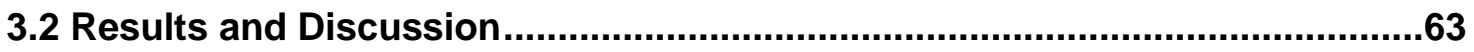

3.2.1 The shape of optically-stimulated serotonin release is frequency dependent63

3.2.2 The amount of serotonin released is frequency dependent...........................64

3.2.3 The amount of serotonin release is dependent on the number of stimulation

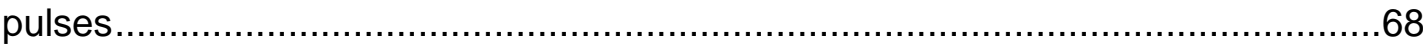

3.2.4 Serotonin release and uptake kinetics following pulsed optical stimulation

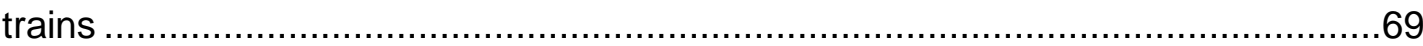

3.2.5 The effects of uptake inhibition on optically stimulated serotonin response...71

3.2.6 Dopamine release and uptake kinetics following pulsed optical stimulation

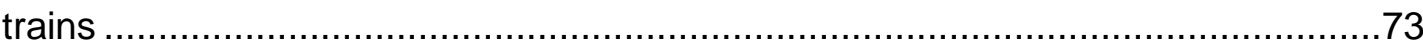

3.2.7 Kinetics values compared to other studies..............................................74

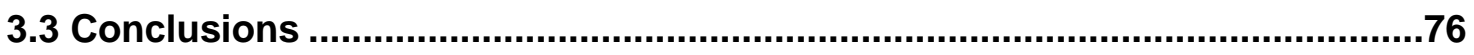

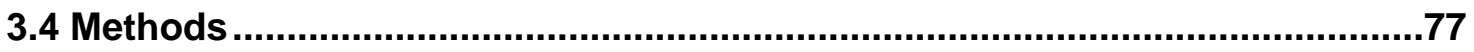

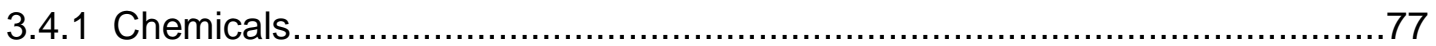

3.4.2 Electrochemical Measurements ............................................................

3.4.3 Preparation of Ventral Nerve Cords ........................................................77

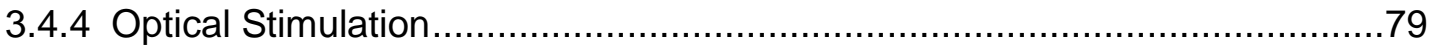

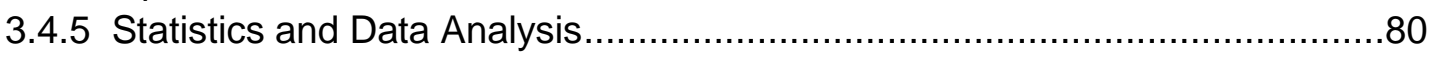

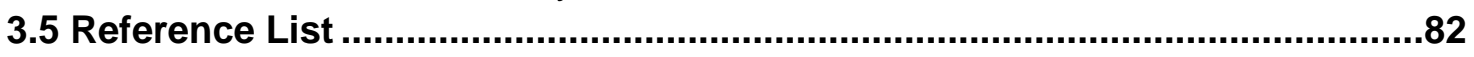

Chapter 4: Characterization of dopamine releasable and reserve pools in Drosophila larva using ATP/ P2X ${ }_{2}$ mediated stimulation ............................. 85

Abstract

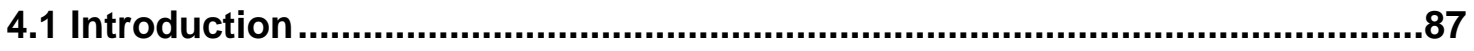

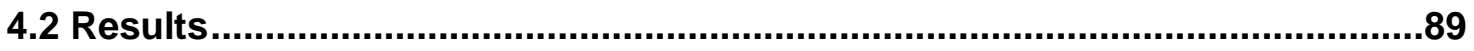

4.2.1 Characterizing ATP/P2X $\mathrm{X}_{2}$ mediated dopamine release in Drosophila.............89

4.2.2 Effect of stimulation intervals on dopamine release ...................................91

4.2.3 Effect of synthesis inhibition and reuptake inhibition on stimulated release....94

4.2.4 Investigating potential cocaine-activated reserve dopamine pool in Drosophila

.

4.3 Discussion.

.99

4.3.1 The releasable dopamine pool is maintained by both synthesis and reuptake

in

4.3.2 There is no cocaine-activated reserve dopamine pool in Drosophila............101

4.4 Conclusions …..........................................................................................102

4.5 Methods

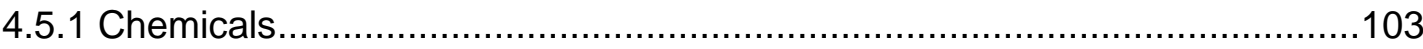

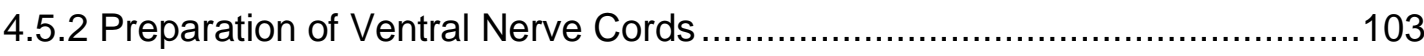

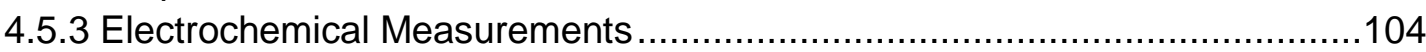

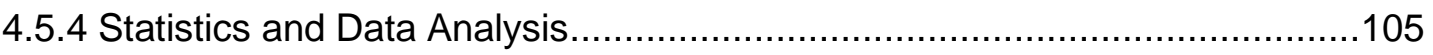




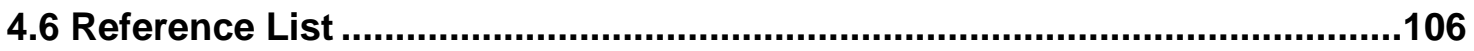

Chapter 5: Conclusions and Future Directions ........................................ 108

5.1 Carbon nanotube-based microelectrodes for in vivo use.............................109

5.2 Future studies in Drosophila............................................................................111

5.3 Final Remarks ......................................................................................113

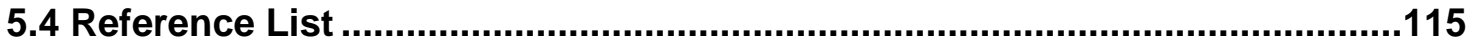




\section{List of Figures}

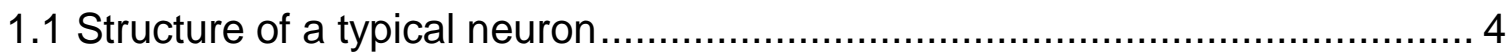

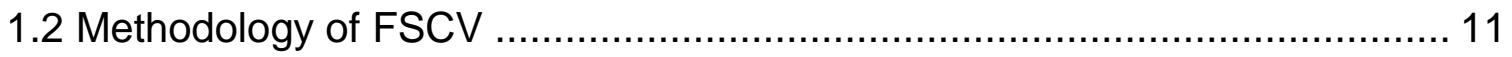

1.3 Modified waveform for serotonin detection ........................................... 12

1.4 SEM images of two types of carbon-fiber microelectrode........................... 14

1.5 A selection of surface functional groups found on carbon surfaces ............. 15

1.6 Biosynthetic pathways for octopamine, dopamine, serotonin and histamine 22

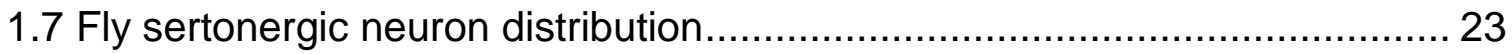

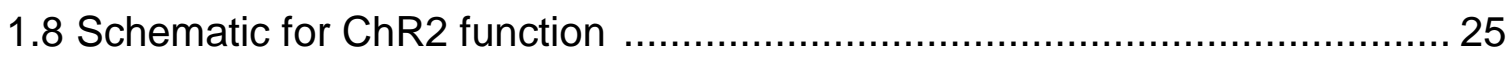

1.9 Schematic for the GAL4-UAS system in Drosophila................................. 26

2.1 Cyclic voltammogram and current vs. time trace of $1 \mu \mathrm{M}$ dopamine at electrodes modified with $0.1 \mathrm{mg} / \mathrm{ml} \mathrm{CNT}$ and $30 \mathrm{~min}$ assembly time................. 39

2.2 Comparison of a bare and a SWCNT forest modified disk carbon-fiber microelectrode for the detection of $1 \mu \mathrm{M}$ dopamine ....................................... 40

2.3 Comparison of different coatings on the oxidation current of $1 \mu \mathrm{M}$ dopamine

2.4 EDS spectroscopy comparing Nafion and Nafion/ $\mathrm{FeO}(\mathrm{OH})$ bilayer electrode

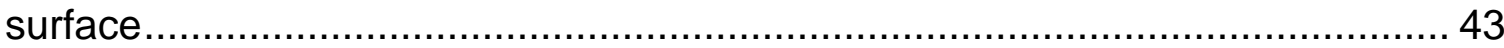

2.5 Scanning electron micrographs of electrodes ........................................... 44

2.6 Detection of different neurochemicals at SWCNT forest electrodes ............. 46

2.7 Comparison of bare and SWCNT forest modified electrodes at higher

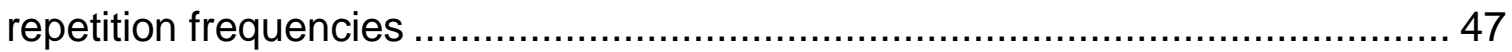

2.8 Measuring different concentrations of dopamine at $10 \mathrm{~Hz}$ and $90 \mathrm{~Hz}$ frequencies

2.9 Comparison of bare and SWCNT forest modified electrodes for dopamine

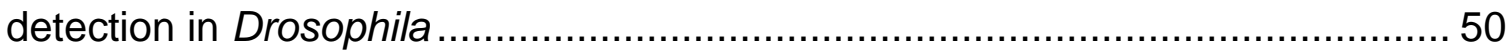

2.10 TEM image of shortened single-walled carbon nanotubes ........................ 54

3.1 Representative serotonin release evoked by different stimulations .............. 64

3.2 Frequency dependency of stimulated serotonin release ...........................6 66

3.3 Pulse width dependency of stimulated serotonin release .......................... 67 
3.4 Effect of pulse number on stimulated serotonin release ............................ 69

3.5 Kinetic modeling of pulsed optically stimulated serotonin release ............... 71

3.6 Effects of serotonin uptake inhibitor fluoxetine ..................................... 73

3.7 Dopamine release evoked by pulsed optical stimulations .......................... 74

3.8 The effect of illumination power on stimulated serotonin release ............... 80

4.1 Characterization of ATP evoked dopamine signal in P2X flies................... 90

4.2 Evoked peak dopamine concentration varies with the amount of pressure-

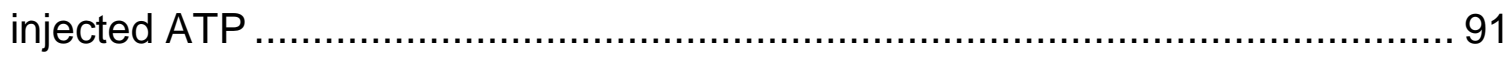

4.3 Dopamine release during stimulations repeated at different intervals .......... 92

4.4 Effect of cocaine and 3-iodotyrosine on repeated stimulations.................... 96

4.5 Comparison of drug effect between $1 \mathrm{~min}$ and 5 min intervals ................... 97

4.6 Effect of cocaine on dopamine release after repeated stimulations in the presence of a synthesis inhibitor .................................................................... 99 


\section{List of Tables}

2-1 Comparison of anodic and cathodic peak currents, peak separation and rise time values for $1 \mu \mathrm{M}$ dopamine between bare and SWCNT forest electrodes .. 41 2-2 Average oxidation currents for different neurochemicals at bare and SWCNT

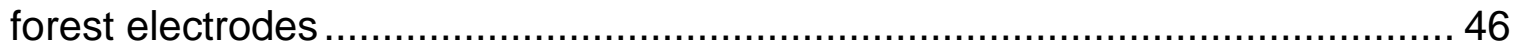

4-1 Statistics for dopamine release during stimulations repeated at different

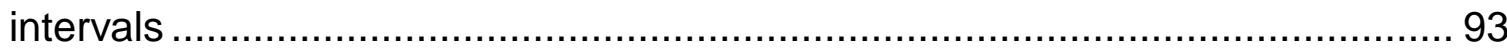

4-2 Statistics for cocaine and 3-iodotyrosine on repeated stimulations ............. 96

4-3 Comparison of drug effect between $1 \mathrm{~min}$ and 5 min intervals ................... 97 


\section{Acknowledgements}

I would like to thank my PhD advisor, Dr B. Jill Venton, who guided me to think as a scientist and gave me huge help on my writing. I'm really grateful for her consistent and illuminating instruction, her encouragement and her patience. Her passion into science truly inspired me. I also want to thank all my labmates. Their support and friendship have made the five years of my doctoral life to be an enjoyable experience.

Thanks to the committee members of my PhD defense, Dr Cafiso, Dr Columbus and Dr Condron for giving me the chance to present my work.

Special thanks to my family. Thanks to my husband Yifei for your company. You are always here whenever I am frustrated or tired. I am lucky to have you in my life. Thanks to my parents, even though you are not around, your support and encouragement never stopped. Thanks to my mother-in-law for taking care of my baby and giving me time to study. Thanks to my baby Zoe, you make me better and stronger. 
Chapter 1

Introduction 


\section{Chapter 1: Introduction}

In the search for drugs to treat disorders of the central nervous system, system biology is emerging as a very important area. By placing a specific molecular within a given biological system, the complex interactions between the molecular and its targets which lead to changes in behavior or development can be monitored. Such approach has become an important avenue of central nervous system drug discovery. Drosophila melanogaster has been extensively used as an effective model organism due to its conserved chemical signaling pathways with mammals, as well as the power of sophisticated genetic modifications. While the fly is quite similar in many respects to mammals, there are likely key differences which need to be taken into account when using Drosophila as a model to study fundamental neuropharmacological processes relevant to human diseases. Our lab has concentrated on developing methods to measure neurotransmitter changes in an intact Drosophila larval central nervous system using fast-scan cyclic voltammetry at carbon-fiber microelectrodes. The goal of this work was to gain a better understanding of the basic mechanisms behind serotonin and dopamine signaling in this simple organism.

\subsection{Brief Overview of neurotransmission}

Most of the chemical communication between cells of neuronal networks in the brain involves small molecules as chemical messengers. The process of information flowing from one neuron to another happens at a specific structure called a synapse. Figure 1.1 shows the structure of a typical neuron, which is composed of three main parts: a cell body (often called the soma), dendrites and an axon. The cell body is where the nucleus is located, and the axonal terminals form the presynaptic portion while the cell bodies or the dendrites of other neurons form the postsynaptic portion (Figure 1.1 bottom). The space between the two neurons is the synaptic cleft and is typically tens of 
nanometers in width. Neurons are excitable cells and have a resting membrane potential due to ionic concentration gradients across the cell membrane. An action potential is produced when the neuron is stimulated and propagates down the axon to the terminal. The signal is passed to the targeted cells through the synapses. Neurotransmitters are small molecules which transmit a message from one neuron to another across a chemical synapse. Before a molecule can be identified as a neurotransmitter, it must satisfy certain criteria ${ }^{1}$ : (i) The molecule must be present in the presynaptic portion. (ii) The precursors and enzymes which are necessary for the synthesis of the molecule must be present in the presynaptic portion. (iii) The molecule must be released in response to activation of the presynaptic neuron and must produce a postsynaptic response, and the release should be $\mathrm{Ca}^{2+}$ dependent. (iv) Specific receptors for the molecule binding to should be present on the postsynaptic membrane. (v) Mechanisms for inactivation of the molecule must be present. Neurotransmitters are synthesized and stored in vesicles in the presynaptic element. The possibility of a neurotransmitter being released to the synaptic cleft is very low in the absence of presynaptic activity, but increases strongly when the presynaptic neuron is depolarized by an action potential which caused the elevations of intracellular $\mathrm{Ca}^{2+}$ concentration. Once released, the neurotransmitters can interact with receptors, diffuse out of the synapse, and be taken up by the transporters or deactivated by certain metabolic pathways. 


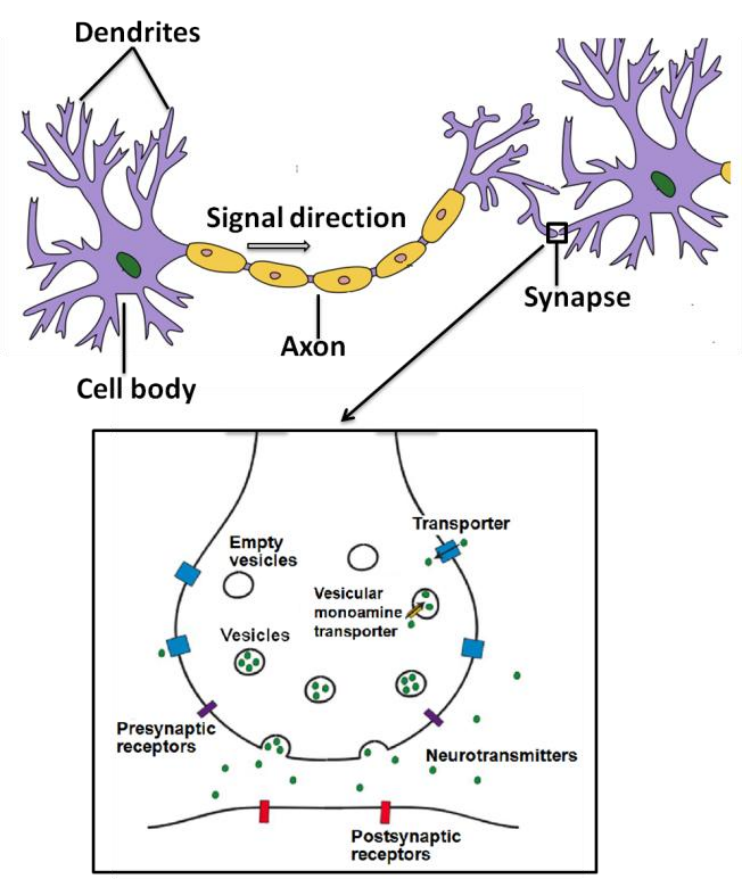

Figure 1.1 The top shows the structure of a typical neuron. Dendrites receive input signal from other neurons, the signal is propagated along the axon, and output to other targeted cells at the axon terminals. The box at the bottom is an enlarged view of a synapse structure. The neurotransmitters are synthesized and packaged into vesicles. Upon an action potential, vesicles release neurotransmitters into the synapse, where they can interact with receptors, diffuse out of the synapse, or be taken up by the transporters. The neuron structure was adapted from website of Wikipedia.

Based on their chemical nature, neurotransmitters can be divided into several categories, including amino acids, monoamines, neuropeptides and some other neurochemicals like acetylcholine (ACh). The significance of developing sensors to track extracellular neurotransmitter changes in the brain is that by following the temporal fluctuations of these signaling molecules, chemists are able to explain how animal behavior is chemically regulated and determine the neurobiological mechanisms behind neurogeneative diseases. For example, dopamine is a neurotransmitter of interest in the catecholamine family. Dopamine is involved in a number of functions in the brain such as regulation of motor behavior, reward, addiction, appetite, arousal, learning and memory. In addition, dysfunction of dopaminergic neurotransmission is related to diseases such as Parkinson's disease, attention-deficit hyperactivity disorder (ADHD), 
and schizophrenia. Another important neurotransmitter is serotonin, an indolamine molecule, which regulates multiple functions in the brain. Serotonin signaling plays a key role in the regulation of development, mood and behavior and is of special interest because it is a contributor to feelings of well-being and happiness. Drugs which modulation of extracellular serotonin levels are used in treatment for several mental disorders including depression, generalized anxiety disorder and social phobia.

\subsection{Electrochemical measurements of neurotransmitters in vivo}

\subsubsection{Requirements for in vivo electrochemical sensor}

Electroanalytical techniques have been widely developed and applied to investigate many neurotransmitters and their metabolites which are electrochemically active. Such techniques have led to a better understanding of neurochemical events ranging from exocytosis of isolated cells to in vivo neurotransmission. ${ }^{2,3}$ However, the brain is a challenging environment for chemical sensing. There are several requirements to make effective electrochemical measurements in vivo. First, the electrochemical sensor should have enough sensitivity for the detection of targeted species in its physiological concentration range. For instance, low concentrations of neurotransmitters from tens of nanomolar to micromolar are released upon various stimulations ${ }^{4}$ and the binding affinities of many neurotransmitter receptors range from nanomolar to micromolar. ${ }^{5,6}$

Second, low concentrations of analytes must be detected in the presence of large amount of interferences, which are electrolyzed at similar potentials and give similar response, obscuring the signals for the analytes. For example, the oxidations of ascorbic acid and 3,4-dihydroxyphenylacetic acid (DOPAC) occur in the same voltage range as that of catecholamines and the problem is particularly aggravating because 
these interferences are present at concentrations 100- to 1000-fold greater than that of catecholamine neurotransmitters in the extracellular fluid. ${ }^{7}$ Similarly, uric acid and 5hydroxyindoleacetic acid (5-HIAA) could interfere the electrochemical detection of serotonin. In addition, various processes inherent to the biological system which could change surface properties of the sensor and affect the sensor response, should also be considered. For example, electrical stimulation has been used for evoking neurotransmitter release in mammalian brain but could cause a alkaline $\mathrm{pH}$ shift due to changes in blood flow. ${ }^{8}$ The $\mathrm{pH}$ shift can interfere with detection of many neurotransmitters such as dopamine by altering background charging current in many electroanalytical techniques. ${ }^{9}$

Another important aspect for in vivo measurement is temporal resolution. Many neurobiological changes occur on the order of millisecond scale. For example, the dopaminergic neurons fire action potentials in two patterns, tonic and phasic. ${ }^{10}$ The tonic firing happens at low frequencies ranging $2-5 \mathrm{~Hz}$, resulting in a steady basal level of extracellular dopamine concentration, which is estimated to be several nanomoles by using microdialysis. ${ }^{11,12}$ The phasic firing occurs at higher frequencies of $15-100 \mathrm{~Hz}$, resulting in a transient increase of the extracellular dopamine concentration to a level much higher than the basal level, which is estimated to be from ten of nanomoles to as high as micromole..$^{4,13,14}$ The phasic neuronal firing happens upon various stimuli and after dopamine is released, it is rapidly cleared by diffusion and uptake, maintaining the relative steady basal level. To examine the mechanisms of neurotransmitter release and clearance, electroanalytical techniques should have fast temporal resolution.

Last but not least, the sensors for measuring neurobiological events should be small enough to cause minimal tissue disturbance. Sensors with enough spatial resolution could enable the sampling of discrete regions of the brain, allowing for the exploration of homogeneity of a specific area. In addition, for direct measurement in 
small biological samples like the fly brain, only microelectrodes with enough spatial resolution can be used. ${ }^{15-17}$ Thus, to effectively monitor neurochemical events in vivo, electroanalytical techniques should be have sufficient sensitivity, good chemical selectivity, and high temporal and spatial resolution.

\subsubsection{Fast-scan cyclic voltammetry with carbon-fiber microelectrode}

Fast-scan cyclic voltammetry (FSCV) is an electrochemical technique which has been widely used in field of neuroscience to detect electroactive molecules such as monoamine neurotransmitters. FSCV has a limit of detection in the tens of nanomolar for catecholamine neurotransmitters like dopamine ${ }^{10}$ and provides a cyclic voltammogram (CV) fingerprint for the species identified. The temporal resolution of FSCV is in the subsecond range, thus allowing real-time measurement of extracellular neurotransmitter changes in vivo. The most common sensor coupled with FSCV is carbon-fiber microelectrode, which offers micrometer-dimension spatial resolution. Combing all these qualities, it is the most suitable technique currently to investigate the rapid events associated with neurotransmission in vivo.

\subsubsection{Basic electrochemistry}

When a potential is applied to the electrode, two types of processes occur at the electrode surface. One type comprises redox reactions in which electrons are transferred across the electrode-solution interface. Electron transfer accompanies oxidation or reduction of the analyte. This process is called faradaic process and governed by Faraday's law, i. e. the amount of chemical reaction caused by the flow of current is proportional to the amount of electron passed. Thus, the number of moles of analytes which react can be calculated using the faradaic current (Equation 1). ${ }^{18}$ 
$\mathrm{Q}=\mathrm{nF} \Delta \mathrm{N}$

Equation 1.1

$\mathrm{Q}=$ amount of charge passed across the electrode (in Coulombs),

$\mathrm{n}=$ number of electrons per molecule of reaction,

$\mathrm{F}=$ Faraday's constant $(96,485 \mathrm{C} / \mathrm{mol})$,

$\Delta \mathrm{N}=$ the number of moles which react (in mole)

However, processes such as changes in the structure of electrode-solution

interface or solution composition can occur and produce currents. But no actual electron exchange happens at the electrode-solution interface, thus these processes are called non-faradaic processes and the current produced is called non-faradaic current. When a potential is applied to electrode, a charge gradient is created between the electrode and solution which causes movement of ions through the solution, rearrangement of charged species near the electrode-solution interface until equilibrium is reached. This process is usually called double layer charging of the electrode-solution interface and can be modeled as charging a capacitor. ${ }^{18}$ The charging current is proportional to both the rate of potential change (i. e. scan rate) and the capacitance of the electrode which is proportional to the electrode surface area. ${ }^{18-20}$

\subsubsection{FSCV data collection}

With FSCV, a triangular waveform is typically used for the detection of catecholamine such as dopamine. The methodology of FSCV is illustrated in Figure 1.2. The potential at the working electrode is scanned as a function of time. The electrode is scanned linearly from a negative holding potential to a positive switching potential and then swept back to the holding potential at a high scan rate $(>100 \mathrm{~V} / \mathrm{s})$. The scan rate and potential limits determine the length of the triangle waveform. For the typical conditions for the detection of dopamine, the potential is ramped from $-0.4 \mathrm{~V}$ to $1.0 \mathrm{~V}$, vs a $\mathrm{Ag} / \mathrm{AgCl}$ reference electrode and back at $400 \mathrm{~V} / \mathrm{s}$ (Figure 1.2A) The entire triangle 
scan takes only $7 \mathrm{~ms}$, and is repeated every $100 \mathrm{~ms}$. The electrode is held at the holding potential between scans, and this relatively long time at the negative holding potential allows for adsorption of positively charged analytes like dopamine on the electrode surface, thus increasing sensitivity. ${ }^{7}$ During the forward scan, analytes with oxidation potentials within the range of potential scanned are oxidized and produce an oxidation/anodic current. During the back scan, the oxidized analytes are reduced back to their original form and a reduction/cathodic current is produced. Measurements with FSCV are typically performed in aqueous solutions, thus the holding potential is usually kept above $-0.6 \mathrm{~V}$ to avoid reducing of oxygen while the switching potential is always kept below $1.5 \mathrm{~V}$ to avoid oxidizing water. ${ }^{21}$

As mentioned earlier, when a potential applied to the electrode, both faradaic and non-faradaic processes can happen and produce currents. The double layer charging current is proportional to scan rate. During FSCV, a large background charging current is produced due to the fast scan rate used (Figure 1.2C, black solid line) and the oxidation and reduction currents of electroactive analytes, such as dopamine, can only make a small increase in the background current (Figure 1.2C, red dashed line). Furthermore, the oxidation and reduction of electroactive functional groups on the electrode surface also contributes to the background current. ${ }^{22}$ However, because the background current for carbon-fiber microelectrodes is stable for approximately $90 \mathrm{~s}^{8,23}$, the background current can be digitally subtracted from the total current, leaving only current resulting from oxidation and reduction of the analyte. The resulting background-subtracted cyclic voltammogram has several features which are used to obtain information about the analyte: the potentials at the oxidation peak $E_{\mathrm{p}, \mathrm{a}}$ and the reduction peak $E_{\mathrm{p}, \mathrm{c}}$ are used to identify the detected species; the potential difference between oxidation and reduction 
$\Delta E_{\mathrm{p}}$ is an indicator of electron transfer kinetics; the magnitude of the oxidation peak current $i_{\mathrm{p}, \mathrm{a}}$ indicates the concentration of the analyte (Figure 1.2D).

For the detection of dopamine, during the forward scan, the current begins increasing at $250 \mathrm{mV}$ where the potential is sufficient for the oxidation of dopamine to dopamine-o-quinone. The current continues to increase and reaches a maximum at 600 $\mathrm{mV} .^{22}$ When the dopamine molecule adsorbed on the electrode surface is completely consumed, the oxidation current goes to zero. During the back scan, the reduction current increases as dopamine-o-quinone generated during the forward scan is reduced and the current peaks at $-200 \mathrm{mV}$. The reduction current decays to zero when dopamine-o-quinone adjacent to the electrode is consumed. The reduction current is smaller than the oxidation current because of diffusional loss of the dopamine-o-quinone produced.

A false color plot is used to view all of the data collected and provide information about current, voltage and time simultaneously (Figure 1.2F). Voltage is plotted on the yaxis. For a triangle waveform, the bottom shows the beginning of the waveform (i. e. holding potential), the middle is the switching potential and the top is where waveform ends (i. e. holding potential). Time is shown on the x-axis, and current is represented in color. Green indicates increases in current produced from oxidation, while blue indicates decreases in current produced form reduction. As shown in the example of dopamine detection, a green area centered around $600 \mathrm{mV}$ due to dopamine oxidation to dopamine-o-quinone and a blue area centered around -200 $\mathrm{mV}$ due to reduction of dopamine-o-quinone to dopamine. If a vertical slice of the color plot is taken, a background-subtracted CV is produced at a given time. If a horizontal slice is taken, the current over time changes can be observed and it is usually taken at the oxidation peak 
of the analyte. By in vitro calibrating the electrode, this current vs time curve can be converted to concentration over time trace (Figure 1.2F, right).

(A)

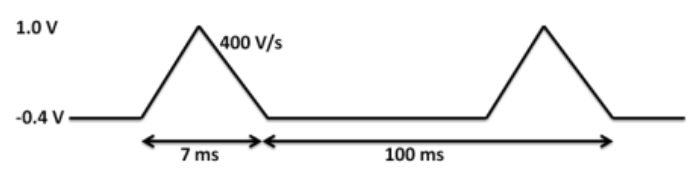

(C)

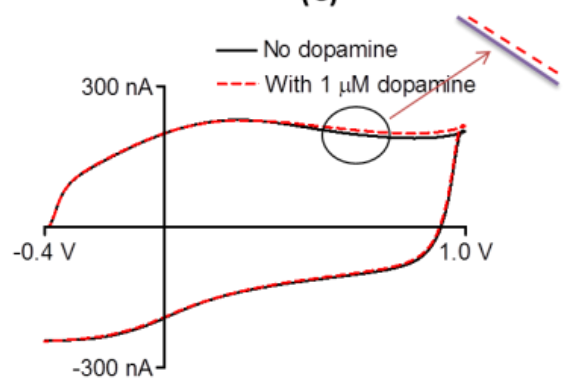

(F)
(B)

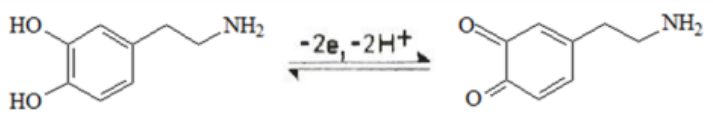

(D)

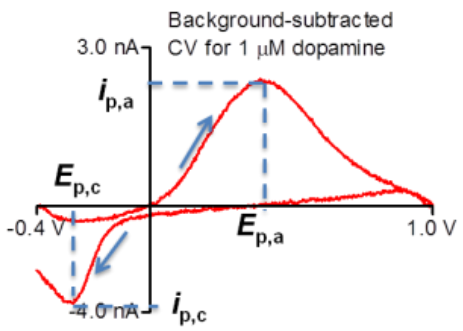

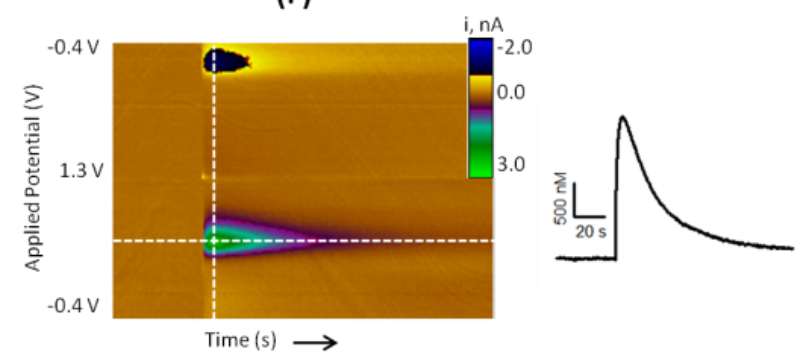

Figure 1.2 (A) Typical triangular waveform for dopamine detection. The applied potential is ramped from $-0.4 \mathrm{~V}$ to $1.0 \mathrm{~V}$, vs a $\mathrm{Ag} / \mathrm{AgCl}$ reference, and back at a scan rate of 400 $\mathrm{V} / \mathrm{s}$. The waveform is repeated every $100 \mathrm{~ms}$. (B) Redox reaction of dopamine. On the forward (anodic) scan, dopamine is oxidized to dopamine-o-quinone. On the reverse (cathodic) scan, dopamine-o-quinone is reduced back to dopamine. (C) Total current collected during the scan. The solid black line is collected in plain Tris buffer and the red dashed line is collected in the presence of $1 \mu \mathrm{M}$ dopamine. Subtracting the black line from the red line results in (D), the background-subtracted CV for $1 \mu \mathrm{M}$ dopamine. Important pieces of information in the CV, including oxidation/anodic peak current $\left(i_{p, a}\right)$, reduction/cathodic peak current $\left(i_{\mathrm{p}, \mathrm{c}}\right)$, oxidation peak potential $\left(E_{\mathrm{p}, \mathrm{c}}\right)$, reduction peak potential $\left(E_{\mathrm{p}, \mathrm{c}}\right)$ as well as the potential difference between oxidation and reduction $\left(\Delta E_{\mathrm{p}}\right)$ provide a chemical fingerprint for the detected species. $(F)$ Color plot showing stimulated dopamine release during in vivo detection. The $x$-axis is time, the $y$-axis is voltage and the current is indicated in color (scale bar on the right). The vertical line shows where the $\mathrm{CV}$ is taken from and the horizontal line shows where the current vs time is taken from. By in vitro calibration, the current vs time trace is converted to concentration changes over time, as shown on the right.

Variation of the scan rate and potential limits affects the selectivity and sensitivity of the detection. ${ }^{24,25}$ Modifications of the triangle waveform have been developed for the 
detection of analyte with different electrochemical properties. Figure 1.3 shows a modified waveform developed for the detection of serotonin. The oxidation products of serotonin and other hydroxyindoles are found to be very reactive and during slow scans, insulating polymers can be formed on the electrode after electrooxidation, thus preventing detection on the subsequent scans. ${ }^{26}$ Furthermore, the primary electroactive products and serotonin itself tend to strongly adsorb on the electrode surface and slow the response time. The modified waveform is scanned from a holding potential of $0.2 \mathrm{~V}$ to $1.0 \mathrm{~V}$, then to $-0.1 \mathrm{~V}$ and back to $0.2 \mathrm{~V}$ at $1000 \mathrm{~V} / \mathrm{s}$ every $100 \mathrm{~ms}$. The scan rate of $1000 \mathrm{~V} / \mathrm{s}$ enables the side reactions which lead to the formation of strongly adsorbed products to be outrun. And the holding potential is kept positive to reduce adsorption. This modified waveform has proved to be sensitive for serotonin over dopamine, and greatly alleviates electrode fouling by serotonin oxidation. ${ }^{27}$

(A)

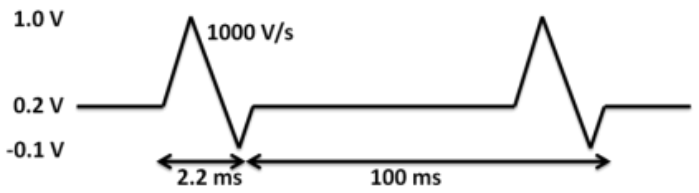

(C)

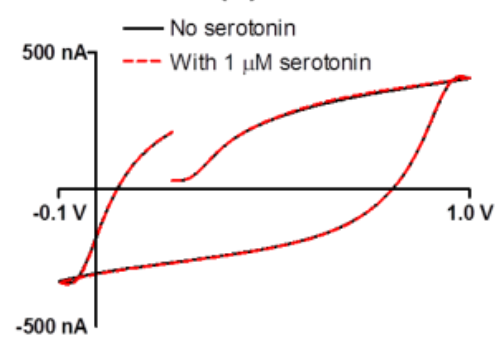

(B)

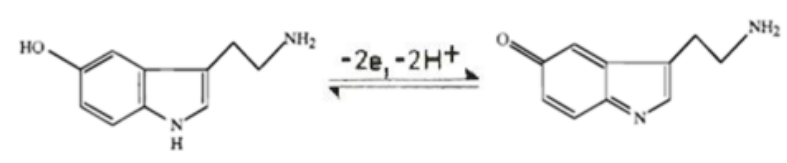

(D)

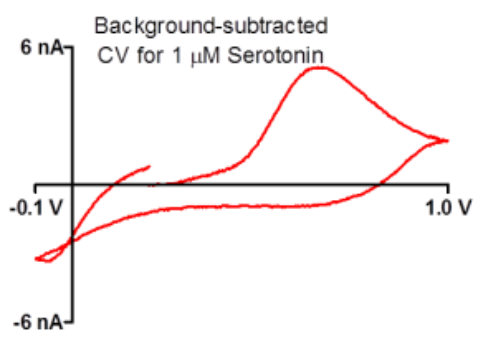

Figure 1.3 (A) Modified waveform for serotonin detection. The applied potential is scanned form $0.2 \mathrm{~V}$ to $1.0 \mathrm{~V}$, then to $-0.1 \mathrm{~V}$ and back to $0.2 \mathrm{~V}$ at $1000 \mathrm{~V} / \mathrm{s}$ every $100 \mathrm{~ms}$, to improve sensitivity and to alleviate electrode fouling. (B) The redox reaction of serotonin. (C) Total current collected under the modified waveform without (black line) and with $1 \mu \mathrm{M}$ serotonin (red line). (D) Background-subtracted CV for $1 \mu \mathrm{M}$ serotonin. 


\subsubsection{Carbon-fiber microelectrode}

Carbon-fiber microelectrodes (CFMEs) are currently the most commonly used electrode with FSCV. There are two common types of CFMEs based on the fabrication process. One type is cylindrical microelectrode (Figure 1.4, left). The cylindrical CFMEs are made by inserting a single carbon-fiber into a glass capillary, then heating and pulling the capillary into two taped electrodes. The remaining space between the fiber and the glass is sealed with epoxy. The carbon-fiber is trimmed to form an electrode. The exposed carbon-fiber is the electroactive area and is $50 \sim 100 \mu \mathrm{m}$ in length for a typical cylindrical microelectrode. The other type is disk microelectrode (Figure 1.4, right) and its fabrication is quite similar to that of the cylindrical CFMEs. After the capillary is pulled into two taped electrodes, the electrode is trimmed at the glass and then epoxied to seal the space. After the epoxy, the electrode is cut again to make a net and flat tip, and the tip is polished on a polishing wheel at a $25-45^{\circ}$ angle to expose an elliptical electroactive surface. The cylinder CFMEs have larger surface area, thus providing greater sensitivity, and are preferred for in vivo experiments. ${ }^{28}$ However, the small surface area of disk CFMEs grants them better spatial resolution, and is suitable for measurements where high spatial resolution is required. Furthermore, with some electrode modification methods, which will be discussed in the next section, the sensitivity of disk CFMEs can be greatly improved without changing the geometrical surface area. 


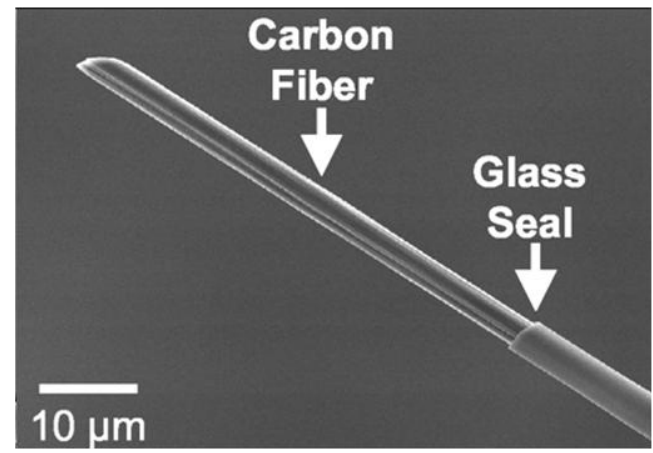

cylindrical

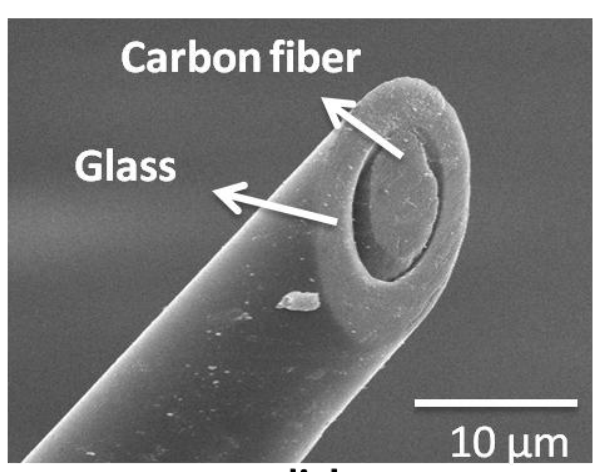

disk

Figure 1.4 Scanning electron microscopic images of a cylindrical (left) and a disk (right) carbon-fiber microelectrode. A $7 \mu \mathrm{m}$ diameter T-650 carbon-fiber is placed inside a pulled glass capillary and trimmed to form a cylinder electrode with a typical length of $50 \sim 100 \mu \mathrm{m}$. The space between the fiber and glass is sealed by epoxy. For the disk electrode, the fiber was trimmed at the glass and the space is sealed by epoxy. The disk electrode was polished at a $30^{\circ}$ angle to create an elliptical active area. The image of cylindrical electrode is taken from D. L. Robinson et al. (2003) ${ }^{29}$

The carbon-fibers are made by heating a carbon-based precursor to a high temperature of several hundred or thousand degrees Celsius. The polymer is spun and pulled into a fiber, resulting in higher conductance along the length than at the edge..$^{30,31}$ Two typical carbon-fiber precursors are polyacrylonitrile (PAN) or pitch, which is a byproduct of petroleum processing. When the temperature goes higher than $300^{\circ} \mathrm{C}$, heteroatoms such as oxygen and nitrogen decompose out of the material causing carbonization, and above $1700^{\circ} \mathrm{C}$, the material becomes increasingly sp $\mathrm{s}^{2}$-hybridized known as graphitization. ${ }^{31}$ With greater heat treatment temperatures, the final product contains fewer heteroatoms and is more conductive. A wide variety of carbon-fibers with different precursors and conductivities are now commercially available, such as the PAN-based T-650 fibers, which are the most commonly used fibers for in vivo measurements of FSCV.

Same as other forms of conductive carbon materials such as highly-ordered pyrolytic graphite (HOPG) and glassy carbon (GC), the basic structure of carbon-fibers is composed of layers of $\mathrm{sp}^{2}$-hybridized graphitic sheets organized at varying degrees of 
order. ${ }^{32}$ The extended benzene-based ring sheet forms the basal plane and is chemically stable in aqueous solution, while the terminating ends of these sheets form the edge planes. At the edge plane, carbon atoms have open orbitals and form bonds with oxygen-containing functional groups such as hydroxyl groups, ketones, and carboxyl groups and these sites are often referred to as "defect sites"(Figure 1.5). ${ }^{7}$ It is commonly accepted that the edge planes are more electrochemically active because these defect sites are important sources of electron transfer and act as adsorption sites for some surface-dependent species. ${ }^{32-34}$ Electrode modifications such as flame etching ${ }^{35}$ and electrochemical overoxidation ${ }^{21}$ have shown to increase defect sites and therefore increase electrode sensitivity.

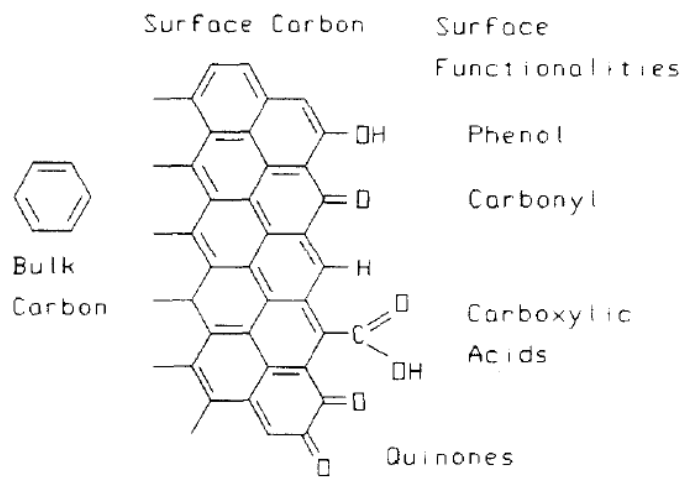

Figure 1.5 A selection of surface functional groups found on carbon materials. Taken from K. T. Kawagoe et al. (1993) ${ }^{7}$

The CFMEs provides high spatial resolution with minimized tissue damage when used for in vivo measurements with FSCV. Furthermore, as mentioned earlier, the background charging current is the most difficult obstacle to overcome with FSCV detection because of the large scan rate. However, the double layer charging current is also proportional to the electrode surface area. The small surface area of CFMEs helps to reduce the difference between faradaic and non-faradaic currents, thus allowing fast scan rates to be used. In addition, microelectrodes detect significantly less ohmic drop comparing to traditional bulk carbon electrodes. The ohmic drop is the product of the 
working electrode current and solution resistance and causes a reduction to the applied potential at the working electrode. Conventional electrolysis setups used three electrodes: a working electrode, a reference electrode and an auxiliary electrode which is used to compensate for the ohmic drop. With CFMEs, the ohmic drop can be disregarded and the auxiliary electrode is not used in FSCV detection. Finally, the carbon-fiber material has more resistance to drift than metal electrodes when exposed to biological tissue, and thus is more suitable for in vivo use. ${ }^{7}$

\subsubsection{Microelectrode modifications}

The structure and chemical composition of carbon electrode surface is vitally important to electrochemical detections, particularly those involving electrocatalysis or selectivity. To improve the sensitivity and selectivity of carbon-fiber microelectrodes, many treatments have been developed to change the surface chemistry. In this section, three commonly used modifications are discussed in detail, including overoxidation, polymer coating and carbon nanotube modification.

The sensitivity of CFMEs can be increased by electrochemical treatments. For example, pretreatment of carbon-fiber microelectrodes by repetitive excursions to +3.0 V vs $\mathrm{Ag} / \mathrm{AgCl}$ at $70 \mathrm{~Hz}$ could increase the sensitivity for dopamine. ${ }^{36,37}$ Another strategy is applying an extended waveform (1.4 potential limit vs $\mathrm{Ag} / \mathrm{AgCl}) .{ }^{21}$ The use of the extended waveform showed substantial increases in sensitivity toward the detection of catechols. ${ }^{38}$ Studies with AFM monitoring changes in carbon surface morphology and with XPS tracking surface-localized fluorine atoms proved that excursions to an extended anodic potentials in aqueous solution caused oxidative etching of the carbon surface and constantly renewed the electrochemically active surface. ${ }^{38}$ The increase in sensitivity arose from oxidative etching which resulted in increased surface roughness thus increasing electroacive surface area and increased surface oxides which act as 
adsorption sites for the catechols. ${ }^{21,38}$ Similar results have also been achieved by flameetching, which fabricated electrode tip with diameter of 1 to $3 \mu \mathrm{m}$ and nanometer-scale surface features. ${ }^{35}$ However, even through overoxidation of the CFME surface either by electrochemical etching or flame-etching improves electrode sensitivity, the time response for cationic neurotransmitter is slower than bare electrode. Electrode overoxidation adds oxide groups and promotes adsorption, resulting slower temporal response..$^{21,38}$ Thus, with such modifications, there is always a trade-off between sensitivity and fast measurements of adsorbed species.

Another approach to improve microelectrode selectivity and sensitivity is to coat the electrode with an ion exchange polymer, such as Nafion and overoxidized polypyrrole. Nafion is a perfluorinated cation exchange polymer. Nafion can form a memmbrane on microelectrode etither by dip-coating or electrodeposition method. ${ }^{39,40}$ The memmbrane can exclude anions from electrode surface and accumlated cations, thus improving senstitivty for cationic neurtoransmitters and enhances selectivity over anion interferences. Furthermore, Nafion-coated microelectrodes has been used in the mammalian brain for the detection of serotonin and proved to alleviate electrode fouling by serotonin oxidation. ${ }^{41}$ Although Nafion provides increased sensitivity and selectivity, it also distort the temporal response of the electrode. ${ }^{40,42}$ The analyte needs to diffuse through the polymer to the get to the electrode surface to be detected, thus slowing the temporal response of the electrode. A study showed the permeation of dopamine at electrode with the ion exchange polymer thinner than $200 \mathrm{~nm}$ is sufficiently fast and the distortion of the time response is not apparent, but the increase in sensitivity is modest. ${ }^{39}$ However, with the commonly used dip-coating procedures, it is hard to coat a uniform film at cylindrical electrode surface. Polypyrrole is a postive charged conducting polymer and upon overoxidation, it loses conductivity and charge. ${ }^{43,44}$ Overoxidation also results in addition of carbonyl and carboxylic groups to this polymer. ${ }^{45-47}$ These functional 
groups could attract cations like dopamine and reject anions such as ascorbate and DOPAC, thus improving electrode sensitivity and selectivity. Overoxidized polypyrrole films have been proposed as an alternative to Nafion polymer as it can easlily coat cylindrical electrodes. ${ }^{48}$ The electrostatic of this type of film is not as strong as the Nafion films, and it was initially proposed to have less of an effect on temporal solution. ${ }^{49}$ However, detection with FSCV at the overoxidized polypyrrole film modified microelectrodes is slower than at bare electrodes. ${ }^{39}$

Carbon nanotubes (CNTs) are a new class of material which have a profound impact on a wide range of applications. Recently, many researchers have demonstrated that CNTs have good properties in the field of electrochemistry. The basic structure of CNT can be thought as seamless rolled up single or multiple layers of graphene sheets. CNTs are generally categorized into single-walled carbon nanotubes, which are considered as a single rolled up graphene sheet, and multi-walled carbon nanotubess which are two or more rolled up graphene sheets. ${ }^{32}$ Such structue results in many inherently properties of CNTs, such as enhanced electronic properties, a large edge/basal plane ratio, and rapid electron kinetics. ${ }^{50,51}$ CNT-based electrodes have shown enchance electrochemcial performance including higher sensitivities, lower limits of detection and faster electron transfer kinetics than traditional used carbon electrodes. ${ }^{52,53}$ The CNT ends have similar properties as graphitic edge planes and are oxide functionalized during some purification process. ${ }^{32,34,54}$ Thus the ends of CNTs have been proposed to be the best sites for electron transfer and for cationic analytes adsorption. ${ }^{24,34,55}$ CNT-modified CFMEs show increased sensitivty without compromising the temporal response. ${ }^{56,57}$ The CNT-modified microelectrodes are commonly fabricated by dipping CFMEs into a CNT suspension, or a CNT/polymer soultion such as CNT/Nafion soltuon. ${ }^{58-62}$ On one side, the polymer could help to suspend the CNTs, on the other hand, the modified electrodes combine the good properties of both the CNTs 
and the polymer. However, the dip-coating method can only deposit CNT films on the electrode surface with nanotubes randomly distributed, and the reproductibiltiy of this method is relatively low. ${ }^{57}$ Chapter 2 describes a method which could deposit CNTs on the microelectrode surface with controlled orientation and density based on a chemical self-assembly mechanism. The modified electrodes fully benefit from the good properties of CNT ends, and showed greatly improved electrochemcial performance.

\subsection{Drosophila}

\subsubsection{Drosophila as a model organism}

Drosophila melanogaster, the fruit fly, has been extensively used as a model organism in developmental biology and genetics research. Research utilizing Drosophila has made significant contributions to the molecular, cellular and evolutionary understanding of human behaviors. After both the human and Drosophila genomes have been decoded, it was estimated that over $60 \%$ of human genes have functional orthologs in Drosophila. ${ }^{63}$ Flies also exhibit a wide range of complex behaviors relevant to mammals, including circadian rhythms, sleep, learning and memory, courtship, feeding, aggression, grooming, and flight navigation, and the molecular and genetic basis of these behaviors helped to elucidate that in mammalian systems. ${ }^{64,65}$ Recently, research has focused on study of neurotransmitter systems in Drosophila and used this model organism to elucidate the genetic basis for neurodegenerative diseases, cellular mechanisms for disease phenotypes, and to search for treatments for disorders of the central nervous system. ${ }^{66-68}$ Drosophila has neurotransmission pathways largely homologous to mammals. Many neurotransmitter systems including serotonin, dopamine, glutamate, GABA, acetylcholine and histamine are present in Drosophila and involved in physiological processes comparable to mammals. ${ }^{67,69-73}$ Key molecules involved in 
synaptic transmission including synthesis enzymes, receptors, and transporters are also highly conserved between Drosophila and mammals. ${ }^{74-77}$ For example, Drosophila express serotonin transporter and four receptor subtypes that are similar to their human orthologs ${ }^{78}$, making this sample organism useful in studying drugs of abuse and treatment of depression and anxiety, which are associated the extracellular serotonin level. ${ }^{79-81}$ In addition, the phenolamines octopamine and tyramine in Drosophila mediate analogous functions to the adrenergic neurotransmitters norepinephrine and epinephrine in mammals. ${ }^{67,82}$ But in contrast to the situation found in vertebrates, there is not a significant level of monoamine oxidase in Drosophila.

The most attractive aspects of Drosophila as a model organism include the rapid generation time and compact genome size. Flies go through several stages of development and the life cycle is $10-12$ days $25^{\circ} \mathrm{C} .{ }^{67}$ The embryogenesis and first two larval stages each takes 24 hours and the third larval stage two days. At the third stage, the larva crawls out of the food substrate and is called 3rd instar wandering larva (L3W). The 3rd instar larval central nervous system becomes fully developed and persists into the adult stage and thus can be used to study the neurobiology of a fully developed nervous system..$^{83,84}$ The larva undergoes a pupae stage (5-7 days) then into adulthood and a single female fly can lay hundreds of eggs within a few days. This short life span and large number of progeny make it relatively easy to produce mutants with desired genetic manipulations in few weeks, compared to years in rodents. Drosophila has 4 chromosomes, only 3 of which carry the bulk of the genes. The entire Drosophila genome has been sequenced and annotated, and about 14,000 genes are predicted..$^{85} \mathrm{~A}$ variety of sophisticated genetic manipulations have been developed for Drosophila, allowing large-scale screening of mutants as well as specific control of both temporal and spatial expression of any given genetic sequence ${ }^{67} \mathrm{~A}$ number of useful Internetbased sources are now available. For example, the Flybase (http://www.flybase.org), a 
database of the Drosophila genome, contains cross-referenced data regarding all annotated fly genes, phenotypes of mutants, references, and reagents. Several large fly stock centers such as the Bloomington Stock Center in Bloomington, Indiana (http://fly.bio.indiana.edu/) provide thousands of mutant alleles of many genes to researchers. These resources greatly ease large-scale screens of mutants to assess the mechanisms underlying human behaviors and neurodegenerative diseases.

\subsubsection{Neurotransmitter systems in Drosophila}

In Drosophila, there are biogenic amines, which act as significant neuroactive molecules in distinct cell populations of the larval and adult nervous system. Studies have demonstrated the presence of biosynthetic machineries, uptake and degradation mechanisms as well as specific receptors coupled to signaling pathways, suggesting theses biogenic amines serve as neurotransmitters in the Drosophila CNS. ${ }^{65,67}$ Serotonin (5-HT), dopamine (DA), histamine $(\mathrm{HA})$, and octopamine $(\mathrm{OA})$ are the major neurotransmitters in Drosophila and their distributions, metabolic pathways and functions in the fly have been extensively studied. Tyramine primarily acts as the biosynthetic intermediate precursor for octopamine, but evidences of its role in producing physiological/behavioral effects and existence of specific tyramine receptors strongly indicate that it is also utilized as a neurotransmitter in Drosophila. ${ }^{86}$ The biosynthetic pathways of these neurotransmitters are shown in Figure 1. 6. In most instances, immunochemical localization has revealed the stereotypic patterns of neurons containing these neurotransmitters and each of these systems exhibits a pattern of a small number of neurons that are widely distributed in the Drosophila CNS. ${ }^{83}$ 


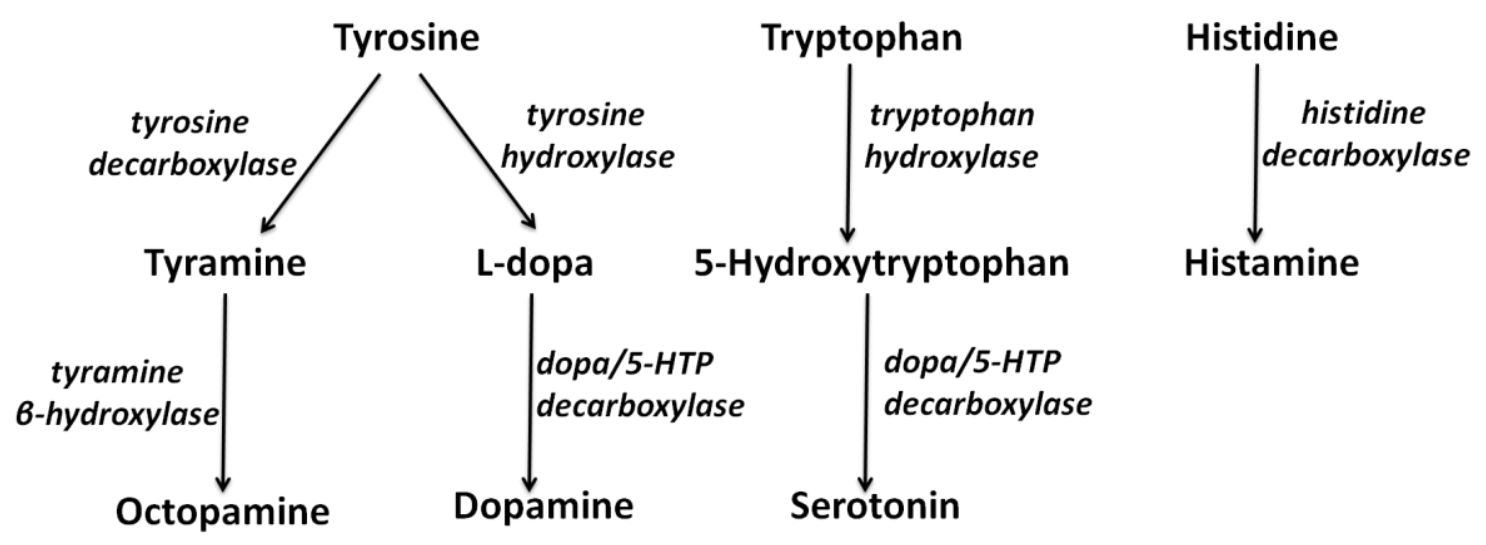

Figure 1.6 Biosynthetic pathways for octopamine, dopamine, serotonin and histamine.

\subsubsection{The serotonergic system in Drosophila}

The development and morphology of the serotonergic system in Drosophila has been well characterized by immunocytochemistry using an antibody directed against serotonin..$^{87,88}$ The serotonergic pattern is composed of a small number of neurons, 84 in larval CNS and 106 in adult $\mathrm{CNS}^{87}$, only a small fraction of the total neurons (approximately $10^{5}$ in the adult fly). ${ }^{89}$ Although few in number, the serotonergic neurons are widely distributed and send projections and varicosities throughout large neuropil regions. In the fly CS, serotonergic neurons are distributed in clusters composed of one to five neurons. ${ }^{87}$ The majority of serotonergic neuronal patterns are preserved throughout the larval stage, undergoing reorganized circuitry into the adult stage during pupation. $^{83}$

Figure $1.7(A)$ shows a photomicrograph of the distributions of serotonin immunoreactive neurons visualized after immunofluorescent staining in a late $3^{\text {rd }}$ instar larva. Figure 1. $7(\mathrm{~B})$ is a schematic tracing adapted from the photomicrograph. The larval CNS is a fused ganglion composed of optic lobes (top) and a ventral nerve cord (bottom). The ventral nerve cord (VNC) is a segmental ganglion analogous to human spinal cord and is composed of the subesophageal, the thoracic and the abdominal 
ganglia. The serotonergic neurons in the VNC are distributed in a bilaterally symmetrical fashion. Each neuron sends its axons across the midline to the contralatral hemisegment where they form extensive intrasegmental arborizations with a highly stereotyped pattern..$^{87,88}$

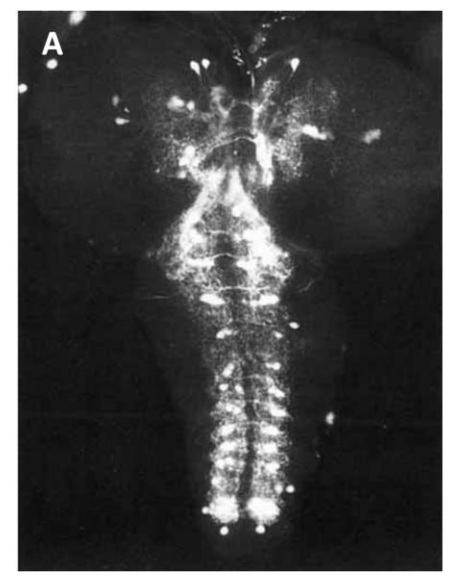

B

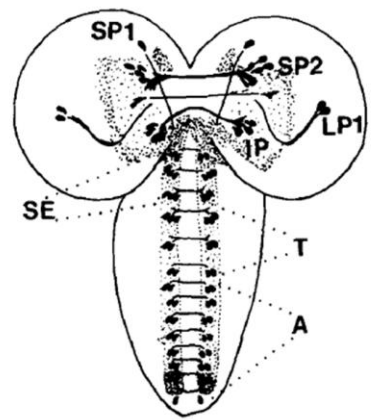

Figure 1.7 (A) A photomicrograph of a whole-mount preparation showing serotonin immunoreactive neurons distribution visualized after immunofluorescent staining of a late 3rd instar larva. (B) A schematic tracing adapted from the photomicrograph in (A). Abbreviations: A, abdominal ganglia; T, thoracic ganglia; SE, subesophageal ganglia; SP, supraesophageal ganglia. Adapted from A.M. Valles et al. (1988) ${ }^{87}$

Serotonin can activate multiple receptor subtypes in Drosophila with distinct expression patterns and signaling properties. Several putative serotonin receptors have been cloned and 4 known serotonin receptor subtypes appear to share sequence similarities with their human orthologs. ${ }^{67}$ In addition, a gene encoding for a serotonin transporter (dSERT) has been found in Drosophila. Pharmacological study of dSERT indicated its sensitivity to cocaine and antidepressants. ${ }^{90}$ The study of dSERT could contribute to the understanding of the structure and function of human serotonin transport and can ultimately inform the design of new selective serotonin reuptake inhibitor (SSRI) and other antidepressants based on transporter inhibition mechanisms.

Several analytical techniques have been developed for measuring serotonin levels in the CNS of Drosophila. One common method is immunohistochemistry for serotonin, which provides imaging of neuronal serotonin tissue levels. This method could 
monitor the intracellular serotonin in real time, but only provides a rough estimate of serotonin content and is not reliable for measuring extracellular serotonin levels. ${ }^{91}$ Another widely used method is homogenization of one or more CNS samples followed by analysis with capillary electrophoresis (CE), high-performance liquid chromatography (HPLC), or mass spectrometry (MS). ${ }^{92,93}$ The analysis of tissue homogenates provides information of total concentration of serotonin and its metabolites, but has little temporal and spatial resolution and cannot be used for real-time measurements. Analytical techniques with the ability to precisely record neurotransmitter changes in the fly sample in real time could acquire direct physiological information, which will bridge the gap between observed behaviors and the chemical signaling pathways that underlie those behaviors. FSCV with carbon-fiber microelectrode has good temporal and spatial resolution, and thus is the ideal candidate technique for the study of neurotransmitter signaling in Drosophila.

\subsubsection{Tools for stimulating neurotransmitter release in Drosophila}

Utilizing Drosophila's genetic palpability, targeted control of specific neuronal activity has been developed with the use of light-activated or ligand-gated ion channels and the yeast GAL4/UAS system.

Channelrhodopsin-2 (ChR2) is a directly light-switched cation-selective ion channel from the green alga Chlamydomonas reinhardtii identified by Nagel et al. ${ }^{94}$ The ChR2 protein is composed of seven transmembrane domains and the chromophore alltrans retinal. Upon blue-light illumination, the all-trans retinal absorbs a photon and undergoes isomerization to 13-cis retinal, resulting in a conformational change of the channel to generate a large permeability for monovalent and divalent cations. ${ }^{95,96}$ The inward flow of cations triggers neuronal depolarization and thus induces exocytosis. When exposed to continuous light illumination, the channel desensitizes to a smaller 
steady-state conductance. ${ }^{94}$ As shown in Figure 1.8B, in a voltage-clamped ChR2-

expressing neuron, large light activated currents is observed rapidly upon stimulation, and the photocurrent decays to a steady-state level, i. e., desensitizes in continuous light.

Boyden et al. expressed ChR2 proteins in mammalian neurons and reported they could drive neuronal depolarization following a series of brief pulses of light. ${ }^{97}$

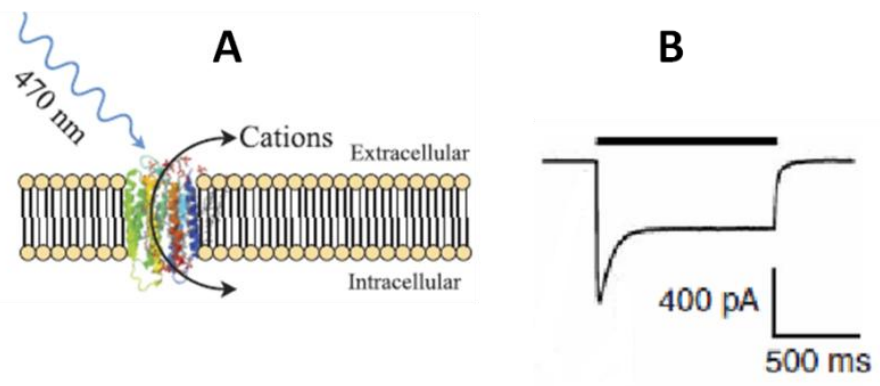

Figure 1.8 (A) Schematic for ChR2 function. Upon blue light stimulation, ChR2 undergoes a conformational change, opening the channel for the passage of cations. Adapted from T. J. Foutz et al. (2011) ${ }^{98}$ (B) Inward current in voltage-clamped neuron expressing ChR2 evoked by $1 \mathrm{~s}$ blue light (indicated by black bar). The channel opens rapidly upon stimulation and then decays to a steady-state with lower conductance. Adapted from E. S. Boyden, et al. (2005) ${ }^{97}$

$\mathrm{P} 2 \mathrm{X}_{2}$ is another non-selective cation channel that is activated by ATP. It is a trimeric channel and each subunit is composed of two transmembrane domains, a large extracellular loop and intracellular $\mathrm{N}$ and $\mathrm{C}$ terminals. ${ }^{99,100}$ It has been suggested that there are three ATP binding sites on the receptor and $\mathrm{P} 2 \mathrm{X}_{2}$ undergoes three sequential ATP binding steps in a cooperative manner. ${ }^{101}$ Once fully liganded, the channel opens rapidly and inward flow of cations leads to neuronal excitation. ${ }^{102}$ Compared to other channels in the P2X family, $\mathrm{P} 2 \mathrm{X}_{2}$ is distinguished by its slow desensitization. It has been proved that currents at $\mathrm{P} 2 \mathrm{X}_{2}$ receptors decline little during sustained ATP application of a few seconds. ${ }^{103,104}$ The Drosophila genome does not encode a $\mathrm{P} 2 \mathrm{X}_{2}$ receptor homology ${ }^{105}$ and previous studies suggest that there are no acute behavioral or physiologic effects of ATP in the absence of transgenic $\mathrm{P}_{2} \mathrm{X}_{2}$ in Drosophila. ${ }^{106,107}$ Thus, $\mathrm{P} 2 \mathrm{X}_{2}$ can be used in Drosophila for targeted neural stimulations. 
Controlling the expression of an ion channel in a specific type of neuron is achieved with the galactosidase-4-upstream activating sequence (GAL4/UAS) system. This system was developed by Brand and Perrimon in 1993 and is composed of two parts: GAL4 and UAS. ${ }^{108}$ GAL4 is a gene which encodes for the yeast transcription activator protein the Gal4 protein and its transcription can be targeted in specific cell type by altering the promoter linked to GAL4. The yeast UAS is an enhancer that Gal4 protein binds to specifically to activate transcription and servers as a promoter for inserted genetic elements. In tissues where Gal4 protein is expressed, UAS is activated and the inserted gene is transcribed. As the example shown in Figure 1.9, the Tph-GAL4 driver line produces flies having Gal4 protein only in neurons where tryptophan hydroxylase (Tph), the enzyme involved in the rate limiting step of serotonin synthesis, is present. Gal4 remains inactive until it binds to an UAS with the Gal4 binding sites. In the UAS-ChR2 responder line, the UAS serves as a promoter for the inserted ChR2 gene. Thus, when the flies are crossed, Gal4 protein is expressed in only serotonergic neurons, where UAS is activated and the inserted ChR2 gene is transcribed. Thus, by genetically expressing $\mathrm{ChR2}$ in serotonergic neurons, serotonin release can be evoked by blue-light stimulation in Drosophila.

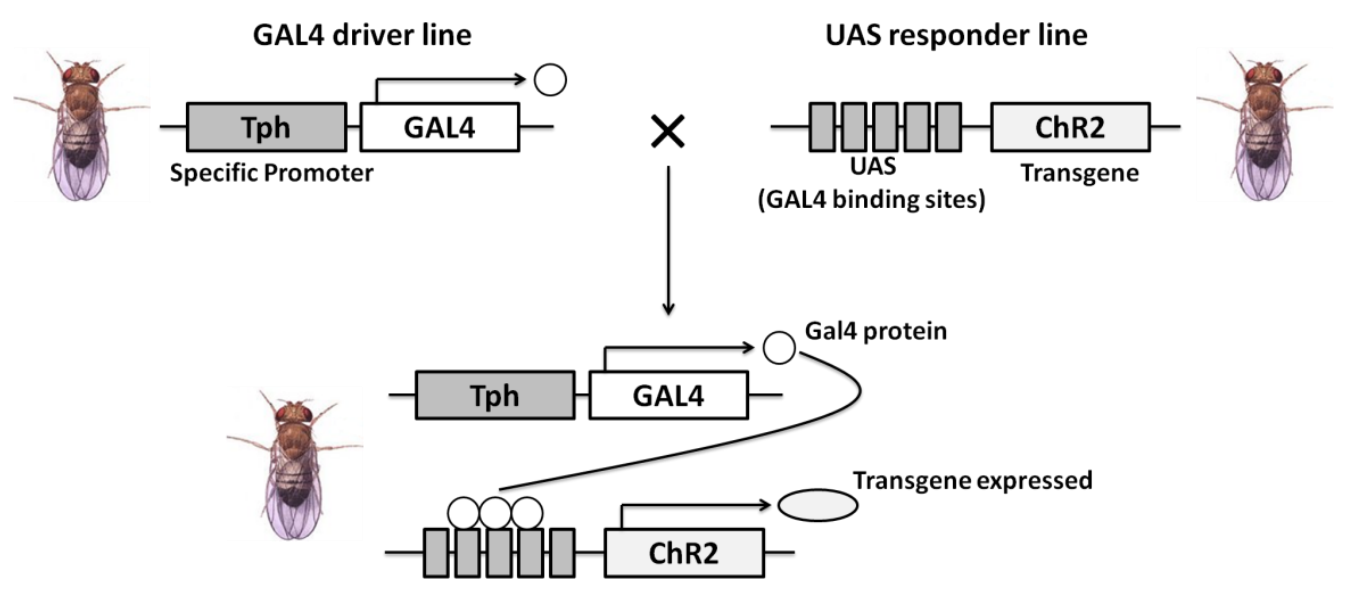


Figure 1.9 Schematic for the GAL4-UAS system in Drosophila. Flies carrying the yeast transfection factor GAL4 under the control of enhancer element of a specific gene, are crossed with flies carrying a transgene linked to the upstream activating sequence (UAS). Once crossed, the progeny express the transgene exclusively in the specific cell or tissue defined by the specific promoter.

\subsubsection{Measurements of neurotransmission in Drosophila with FSCV}

The initial characterization of FSCV for real time measurements of serotonin and dopamine in Drosophila has been performed in previous studies. ${ }^{109-111}$ Results of these studies indicated the basic serotonergic and dopaminergic signaling mechanisms in the fly are analogous to that of mammals. This dissertation describes further development of this method in study of neurotransmitter systems in Drosophila, with some critical technical improvements. Chapter II focuses on developing a highly sensitive microelectrode modified with self-assembled SWCNT forests. The high sensitivity enables improved temporal resolution up to $90 \mathrm{~Hz}$ detection, facilitating enhanced electrochemical measurements of neurotransmitter release in vivo. Chapter III focuses on optimizing stimulation of serotonin release in Drosophila with a pulsed optical stimulation train. The short pulsed stimulations allow for mimicking physiologically neuronal firing patterns and furthermore, the kinetics of optical induced neurotransmitter release can be determined by the same modeling method which has been well established in mammals with electrical stimulations. Chapter IV characterizes ATP/P2X 2 mediated dopamine release and evaluates the roles of synthesis and reuptake in maintaining the releasable dopamine pool in Drosophila. Finally, chapter V discusses the future perspectives in this field. The work discussed here greatly expands the utility of the fruit fly for understanding the underlying neurobiology for neurotransmitter regulation, and explodes the potential for this model organism to aid in drug discovery relevant to human neurodegenerative diseases. 


\subsection{Reference List}

(1) Hammond, C. Cellular and Molecular Neurophysiology: Edition 3; Academic Press: 2008.

(2) Wightman, R. M.; Jankowski, J. A.; Kennedy, R. T.; Kawagoe, K. T.; Schroeder, T. J.; Leszczyszyn, D. J.; Near, J. A.; Diliberto, E. J.; Viveros, O. H. Proc. Natl. Acad. Sci. U. S. A. 1991, 88, 10754-10758.

(3) Millar, J.; Stamford, J. A.; Kruk, Z. L.; Wightman, R. M. Eur. J. Pharmacol. 1985, 109, 341-348.

(4) Venton, B. J.; Wightman, R. M. Anal. Chem. 2003, 75, 414A-421A.

(5) Richfield, E. K.; Penney, J. B.; Young, A. B. Neuroscience 1989, 30, 767-777.

(6) Berke, J. D.; Hyman, S. E. Neuron 2000, 25, 515-532.

(7) Kawagoe, K. T.; Zimmerman, J. B.; Wightman, R. M. J. Neurosci. Meth. 1993, 48, 225-240.

(8) Heien, M. L. A. V.; Khan, A. S.; Ariansen, J. L.; Cheer, J. F.; Phillips, P. E. M.; Wassum, K. M.; Wightman, R. M. Proc. Natl. Acad. Sci. USA 2005, 102, 1002310028.

(9) Venton, B. J.; Michael, D. J.; Wightman, R. M. J. Neurochem. 2003, 84, 373-381.

(10) Wightman, R. M.; Robinson, D. L. J. Neurochem. 2002, 82, 721-735.

(11) Grace, A. A. Neuroscience 1991, 41, 1-24.

(12) Justice, J. B. J. Neurosci. Meth. 1993, 48, 263-276.

(13) Robinson, D. L.; Heien, M. L. A. V.; Wightman, R. M. J. Neurosci. 2002, 22, 10477-10486.

(14) Phillips, P. E. M.; Stuber, G. D.; Heien, M. L. A. V.; Wightman, R. M.; Carelli, R. M. Nature 2003, 422, 614-618.

(15) Makos, M. A.; Kim, Y. C.; Han, K. A.; Heien, M. L.; Ewing, A. G. Anal. Chem. 2009, $81,1848-1854$.

(16) Borue, X.; Cooper, S.; Hirsh, J.; Condron, B.; Venton, B. J. J. Neurosci. Meth. 2009, 179, 300-308.

(17) Vickrey, T. L.; Condron, B.; Venton, B. J. Anal. Chem. 2009, 81, 9306-9313.

(18) Bard, A. J.; Faulkner, L. R. Electrochemical Methods: Fundamentals and Applications, 2nd Edition; Wiley, John \& Sons, Incorporated: 2000. 
(19) Bagotski, V. S. Fundamentals of Electrochemistry, 2nd Edition; Wiley-Interscience, Hoboken, N.J. 2006.

(20) Serway, R. A.; Jewett, J. W. Physics for scientists and engineers ; ThomsonBrooks/Cole, Belmont, CA.: 2004.

(21) Heien, M. L. A. V.; Phillips, P. E. M.; Stuber, G. D.; Seipel, A. T.; Wightman, R. M. Analyst 2003, 128, 1413-1419.

(22) Robinson, D. L.; Venton, B. J.; Heien, M. L.; Wightman, R. M. Clin. Chem. 2003, 49, 1763-1773.

(23) Stamford, J. A. J. Neurosci. Methods 1990, 34, 67-72.

(24) Bath, B. D.; Michael, D. J.; Trafton, B. J.; Joseph, J. D.; Runnels, P. L.; Wightman, R. M. Anal. Chem. 2000, 72, 5994-6002.

(25) Kile, B. M.; Walsh, P. L.; McElligott, Z. A.; Bucher, E. S.; Guillot, T. S.; Salahpour, A.; Caron, M. G.; Wightman, R. M. ACS Chem. Neurosci. 2012, 3, 285-292.

(26) Dayton, M. A.; Ewing, A. G.; Wightman, R. M. Eur. J. Pharmacol. 1981, 75, 141144.

(27) Jackson, B. P.; Dietz, S. M.; Wightman, R. M. Anal. Chem. 1995, 67, 1115-1120.

(28) Venton, B. J.; Wightman, R. M. Anal. Chem. 2003, 75, 414A-421A.

(29) Robinson, D. L.; Venton, B. J.; Heien, M. L.; Wightman, R. M. Clin. Chem. 2003, 49, 1763-1773.

(30) R.L.McCreery in Electroanalytical Chemistry, 17 ed; Marcel Dekker, New York: 1991.

(31) D.D.Edie; J.J.McHugh in Carbon Materials for Advanced Technologies; Pergamon, Amsterdam: 1999.

(32) McCreery, R. L. Chem. Rev. 2008, 108, 2646-2687.

(33) Banks, C. E.; Compton, R. G. Anal. Sci. 2005, 21, 1263-1268.

(34) Banks, C. E.; Davies, T. J.; Wildgoose, G. G.; Compton, R. G. Chem. Commun (Camb. ) 2005, 829-841.

(35) Strand, A. M.; Venton, B. J. Anal. Chem. 2008, 80, 3708-3715.

(36) Gonon, F.; Buda, M.; Cespuglio, R.; Jouvet, M.; Pujol, J. F. Nature 1980, 286, 902-904.

(37) Gonon, F.; Buda, M.; Cespuglio, R.; Jouvet, M.; Pujol, J. F. Brain Res. 1981, 223, 69-80. 
(38) Takmakov, P.; Zachek, M. K.; Keithley, R. B.; Walsh, P. L.; Donley, C.; McCarty, G. S.; Wightman, R. M. Anal. Chem. 2010, 82, 2020-2028.

(39) Kristensen, E. W.; Kuhr, W. G.; Wightman, R. M. Anal. Chem. 1987, 59, 17521757.

(40) Rice, M. E.; Nicholson, C. Anal. Chem. 1989, 61, 1805-1810.

(41) Hashemi, P.; Dankoski, E. C.; Petrovic, J.; Keithley, R. B.; Wightman, R. M. Anal. Chem. 2009, 81, 9462-9471.

(42) Ross, A. E.; Venton, B. J. Analyst 2012, 137, 3045-3051.

(43) Diaz, A. F.; Castillo, J. I. J. Chem. Soc. , Chem. Commun. 1980, 397-398.

(44) Beck, F.; Braun, P.; Oberst, M. Ber. Bunsenges. Phys. Chem. 1987, 91, 967-974.

(45) Ge, H.; Qi, G.; Kang, E.; Neoh, K. G. Polymer 1994, 35, 504-508.

(46) Palmisano, F.; Malitesta, C.; Centonze, D.; Zambonin, P. G. Anal. Chem. 1995, 2207-2211.

(47) Christensen, P. A.; Hamnett, A. Electrochim. Acta 1991, 36, 1263-1286.

(48) Pihel, K.; Walker, Q. D.; Wightman, R. M. Anal. Chem. 1996, 68, 2084-2089.

(49) C.C.Hsueh; A.Brajter-Toth Anal. Chem. 1994, 66, 2458-2464.

(50) Agui, L.; Yanez-Sedeno, P.; Pingarron, J. M. Anal. Chim. Acta 2008, 622, 11-47.

(51) Trojanowicz, M. TrAC Trends in Analytical Chemistry 2006, 25, 480-489.

(52) Gooding, J. Electrochimica Acta 2005, 50, 3049-3060.

(53) Dresselhaus, M. S.; Dresselhaus, G.; Jorio, a. Annu. Rev. Mater. Res. 2004, 34, 247-278.

(54) Liu, J.; Rinzler, A. G.; Dai, H.; Hafner, J. H.; Bradley, R. K.; Boul, P. J.; Lu, A.; Iverson, T.; Shelimov, K.; Huffman, C. B.; Rodriguez-Macias, F.; Shon, Y. S.; Lee, T. R.; Colbert, D. T.; Smalley, R. E. Science 1998, 280, 1253-1256.

(55) Banks, C. E.; Moore, R. R.; Davies, T. J.; Compton, R. G. Chem. Commun (Camb. ) 2004, 1804-1805.

(56) Swamy, B. E.; Venton, B. J. Analyst 2007, 132, 876-884.

(57) Jacobs, C. B.; Vickrey, T. L.; Venton, B. J. Analyst 2011, 136, 3557-3565.

(58) Hocevar, S. B.; Wang, J.; Deo, R. P.; Musameh, M.; Ogorevc, B. Electroanalysis 2005, 17, 417-422.

(59) Swamy, B. E.; Venton, B. J. Analyst 2007, 132, 876-884. 
(60) Jacobs, C. B.; Vickrey, T. L.; Venton, B. J. Analyst 2011, 136, 3557-3565.

(61) Jeong, H.; Jeon, S. Sensors (Basel) 2009, 9, 376.

(62) M.J.Peairs; A.E.Ross; B.J.Venton Anal. Methods 2011, 3, 2379-2386.

(63) Bernards, A.; Hariharan, I. K. Curr. Opin. Genet. Dev. 2001, 11, 274-278.

(64) Greenspan, R. J.; Dierick, H. A. Hum. Mol. Genet. 2004, 13 Spec No 2, R267R273.

(65) Greenspan, R. J.; van, S. B. Trends Neurosci. 2004, 27, 707-711.

(66) Makos, M. A.; Kuklinski, N. J.; Berglund, E. C.; Heien, M. L.; Ewing, A. G. Trends Analyt. Chem. 2009, 28, 1223-1234.

(67) Nichols, C. D. Pharmacol. Ther. 2006, 112, 677-700.

(68) Bier, E. Nat. Rev. Genet. 2005, 6, 9-23.

(69) Schwaerzel, M.; Monastirioti, M.; Scholz, H.; Friggi-Grelin, F.; Birman, S.; Heisenberg, M. J. Neurosci. 2003, 23, 10495-10502.

(70) Li, H.; Chaney, S.; Roberts, I. J.; Forte, M.; Hirsh, J. Curr. Biol. 2000, 10, 211-214.

(71) Kume, K.; Kume, S.; Park, S. K.; Hirsh, J.; Jackson, F. R. J. Neurosci. 2005, 25, 7377-7384.

(72) McClung, C.; Hirsh, J. Curr. Biol. 1999, 9, 853-860.

(73) Bergquist, J.; Sciubisz, A.; Kaczor, A.; Silberring, J. J. Neurosci. Methods 2002, 113, 1-13.

(74) Blenau, W.; Baumann, A. Arch. Insect Biochem. Physiol 2001, 48, 13-38.

(75) Maqueira, B.; Chatwin, H.; Evans, P. D. J. Neurochem. 2005, 94, 547-560.

(76) Coleman, C. M.; Neckameyer, W. S. Arch. Insect Biochem. Physiol 2005, 59, $12-$ 31.

(77) Porzgen, P.; Park, S. K.; Hirsh, J.; Sonders, M. S.; Amara, S. G. Mol. Pharmacol. 2001, 59, 83-95.

(78) Saudou, F.; Boschert, U.; Amlaiky, N.; Plassat, J. L.; Hen, R. EMBO J. 1992, 11, 7-17.

(79) Coppen, A. Br. J. Psychiatry 1967, 113, 1237-1264.

(80) Lasky-Su, J. A.; Faraone, S. V.; Glatt, S. J.; Tsuang, M. T. Am. J. Med. Genet. B Neuropsychiatr. Genet. 2005, 133B, 110-115.

(81) Serretti, A.; Kato, M.; De, R. D.; Kinoshita, T. Mol. Psychiatry 2007, 12, 247-257. 
(82) Monastirioti, M. Microsc. Res. Tech. 1999, 45, 106-121.

(83) Budnik, V.; White, K. J. Comp Neurol. 1988, 268, 400-413.

(84) Sykes, P. A.; Condron, B. G. Dev. Biol. 2005, 286, 207-216.

(85) Celniker, S. E.; Rubin, G. M. Annu. Rev. Genomics Hum. Genet. 2003, 4, 89-117.

(86) Lange, A. B. Gen. Comp Endocrinol. 2009, 162, 18-26.

(87) Valles, A. M.; White, K. J. Comp Neurol. 1988, 268, 414-428.

(88) Chen, J.; Condron, B. G. Dev. Biol. 2008, 320, 30-38.

(89) Benzer, S. Proc. Natl. Acad. Sci. U. S A 1967, 58, 1112-1119.

(90) Corey, J. L.; Quick, M. W.; Davidson, N.; Lester, H. A.; Guastella, J. Proc. Natl. Acad. Sci. U. S A 1994, 91, 1188-1192.

(91) Balaji, J.; Desai, R.; Kaushalya, S. K.; Eaton, M. J.; Maiti, S. J. Neurochem. 2005, 95, 1217-1226.

(92) Makos, M. A.; Kuklinski, N. J.; Berglund, E. C.; Heien, M. L.; Ewing, A. G. Trends Analyt. Chem. 2009, 28, 1223-1234.

(93) Hardie, S. L.; Hirsh, J. J. Neurosci. Methods 2006, 153, 243-249.

(94) Nagel, G.; Szellas, T.; Huhn, W.; Kateriya, S.; Adeishvili, N.; Berthold, P.; Ollig, D.; Hegemann, P.; Bamberg, E. Proc. Natl. Acad. Sci. U. S A 2003, 100, 1394013945.

(95) Yew, J. Y.; Cody, R. B.; Kravitz, E. A. Proc. Natl. Acad. Sci. U. S A 2008, 105, 7135-7140.

(96) Fire, A.; Xu, S.; Montgomery, M. K.; Kostas, S. A.; Driver, S. E.; Mello, C. C. Nature 1998, 391, 806-811.

(97) Boyden, E. S.; Zhang, F.; Bamberg, E.; Nagel, G.; Deisseroth, K. Nat. Neurosci. 2005, 8, 1263-1268.

(98) Foutz, T. J.; Arlow, R. L.; Mclntyre, C. C. J. Neurophysiol. 2012, 107, 3235-3245.

(99) Torres, G. E.; Egan, T. M.; Voigt, M. M. FEBS Lett. 1998, 425, $19-23$.

(100) Roberts, J. A.; Vial, C.; Digby, H. R.; Agboh, K. C.; Wen, H.; Atterbury-Thomas, A.; Evans, R. J. Pflugers Arch. 2006, 452, 486-500.

(101) Ding, S.; Sachs, F. J. Gen. Physiol 1999, 113, 695-720.

(102) North, R. A. Physiol Rev. 2002, 82, 1013-1067.

(103)Brake, A. J.; Wagenbach, M. J.; Julius, D. Nature 1994, 371, 519-523. 
(104)Collo, G.; North, R. A.; Kawashima, E.; Merlo-Pich, E.; Neidhart, S.; Surprenant, A.; Buell, G. J. Neurosci. 1996, 16, 2495-2507.

(105) Littleton, J. T.; Ganetzky, B. Neuron 2000, 26, 35-43.

(106) Lima, S. Q.; Miesenbock, G. Cell 2005, 121, 141-152.

(107) Yao, Z.; Macara, A. M.; Lelito, K. R.; Minosyan, T. Y.; Shafer, O. T. J. Neurophysiol. 2012, 108, 684-696.

(108) Brand, A. H.; Perrimon, N. Development 1993, 118, 401-415.

(109) Borue, X.; Cooper, S.; Hirsh, J.; Condron, B.; Venton, B. J. J. Neurosci. Meth. 2009, 179, 300-308.

(110) Borue, X.; Condron, B.; Venton, B. J. J. Neurochem. 2010, 113, 188-199.

(111) Vickrey, T. L.; Condron, B.; Venton, B. J. Anal. Chem. 2009, 81, 9306-9313. 
Chapter 2

Rapid, sensitive detection of neurotransmitters at microelectrodes modified with self-assembled SWCNT

forests 


\section{Chapter 2: Rapid, sensitive detection of neurotransmitters at microelectrodes modified with self-assembled SWCNT forests}

\section{Abstract}

Carbon nanotube (CNT) modification of microelectrodes can result in increased sensitivity without compromising time response. However, dip coating CNTs is not very reproducible and the CNTs tend to lay flat on the electrode surface which limits access to the electroactive sites on the ends. In this study, aligned CNT forests were formed using a chemical self-assembly method, which resulted in more exposed CNT ends to the analyte. Shortened, carboxylic acid functionalized single-walled CNTs were assembled from a DMF suspension onto a carbon-fiber disk microelectrode modified with a thin iron hydroxide-decorated Nafion film. The modified electrodes were highly sensitive, with 36-fold higher oxidation currents for dopamine using fast-scan cyclic voltammetry than bare electrodes and 34-fold more current than electrodes dipped in CNTs. The limit of detection for dopamine was $17 \pm 3 \mathrm{nM}$ at a $10 \mathrm{~Hz}$ repetition rate and $65 \pm 7 \mathrm{nM}$ at $90 \mathrm{~Hz}$. The LOD at $90 \mathrm{~Hz}$ was the same as a bare electrode at $10 \mathrm{~Hz}$, allowing a 9-fold increase in temporal resolution without a decrease in sensitivity. Similar increases were observed for other cationic catecholamine neurotransmitters and the increases in current were greater than for anionic interferents such as ascorbic acid and 3,4-dihydroxyphenylacetic acid (DOPAC). The CNT forest electrodes had high sensitivity at $90 \mathrm{~Hz}$ repetition rate when stimulated dopamine release was measured in Drosophila. The sensitivity, temporal resolution, and spatial resolution of these CNT forest modified disk electrodes facilitate enhanced electrochemical measurements of neurotransmitters release in vivo. 


\subsection{Introduction}

Carbon-fiber microelectrodes have been extensively used to probe electroactive species in the brain with high spatial resolution and minimal tissue damage. ${ }^{1-5}$ In vivo measurements are challenging because the amount of neurotransmitter is low, as evidenced by the nanomolar to micromolar binding affinities of many neurotransmitter receptors, ${ }^{6,7}$ and many electroacive endogenous intereferents are present in the extracellular fluid. ${ }^{3,5,8}$ Fast-scan cyclic voltammetry (FSCV) is the most popular electrochemical technique for measuring changes in electroactive neurotransmitters and neuromodulator concentrations because it has fast temporal resolution and provides a cyclic voltammogram fingerprint of the species identified. ${ }^{1,3,9}$ Electrochemical detection of many cationic neurotransmitters, such as dopamine, is highly dependent on adsorption to the carbon electrode..$^{8,10,11}$ With FSCV, scans are normally repeated at $10 \mathrm{~Hz}$, as a trade-off between fast measurements and sensitive detection of adsorbed species. ${ }^{12}$ To improve sensitivity and selectivity, many treatments have been developed to change the surface chemistry including polymer coatings ${ }^{13,14}$ and overoxidation of the electrode surface. ${ }^{15,16}$ While these treatments increase the sensitivity of the electrodes, they also slow the time response, because polymer coatings restrict diffusion to the electrode surface and electrode overoxidation adds oxide groups and promotes adsorption. Thus, electrode treatments that increase sensitivity while maintaining high temporal resolution still need to be developed.

Recently, many researchers have demonstrated that carbon nanotubes (CNTs) have good electrochemical properties and can increase the sensitivity and electron transfer of traditional electrodes, such as glassy carbon electrodes. ${ }^{17-20}$ CNT-modified electrodes exhibit faster electron transfer kinetics, reduced electrode fouling, and increased sensitivity and selectivity for adsorption-controlled species. ${ }^{21-23}$ CNT-modified microelectrodes show increased sensitivity without compromising the temporal 
response, ${ }^{22,23}$ an advantage over polymer-coated and electrochemically treated electrodes. The most popular method to deposit CNT films on a microelectrode surface is to dip carbon-fiber microelectrodes into either a CNT suspension or a CNT/polymer solution. ${ }^{21-25}$ Two main drawbacks limit the optimization of this method. First, nanotubes are randomly distributed throughout the CNT films and thus most of the area exposed to the analyte solution is the sidewall of the CNTs. However, the ends of the CNTs are likely to be the best sites for electron transfer because they have similar properties to graphitic edge planes and are oxide functionalized during CNT purification process. ${ }^{26-31}$ Second, a dense and uniform layer of single CNTs is hard to deposit on the electrode surface and large CNT agglomerations are easily formed, causing high noise and low reproducibility. ${ }^{23}$ Therefore, to fully benefit from the CNTs, an electrode modification strategy must be developed to deposit CNTs on the microelectrode surface with controlled orientation and density.

Fabrication of vertically aligned CNTs has also received much attention recently and two main strategies have been developed. ${ }^{32-34}$ One strategy is to directly grow CNTs in an aligned manner through chemical vapor deposition with a solid-phase catalyst deposited on the substrate surface ${ }^{35}$ This strategy can be robust but requires specialized equipment and is less amenable to mass production. Another strategy is to chemically self-assemble vertically aligned CNTs on a substrate with a solution deposition method. ${ }^{36-38}$ The immobilization is based on strong interactions between functional groups at the end of CNTs and the modified electrode surface, and alignment is driven by hydrophobic interactions between the sidewalls of CNTs. Chemical selfassembly does not require specialized equipment so it should be possible to fabricate an ordered, dense coating of CNTs on a microelectrode surface to preferentially expose the ends of the CNTs to the solution. 
In this study, we used a chemical self-assembly mechanism to deposit shortened, carboxylic acid functionalized single-walled CNTs onto a disk carbon-fiber

microelectrode modified with a thin iron hydroxide-decorated Nafion film. ${ }^{37,39}$ These shortened assembled CNT layers are referred to as CNT forests because of their needlelike domains. ${ }^{37}$ The CNT forest modified microelectrodes showed greater than 30 fold increases in current for cations such as dopamine. The time response of the electrodes did not change after coating and the large increases in sensitivity facilitated measurements using faster repetition rates with FSCV. Finally, these CNT forest electrodes were used to detect endogenous dopamine changes in the ventral nerve cord of Drosophila melanogaster and were able to maintain both high sensitivity and rapid measurements in vivo.

\subsection{Results and Discussion}

\subsubsection{Fast-scan cyclic voltammetry of dopamine at SWCNT forest electrodes}

Previous studies have modified larger substrates, such as pyrolytic graphite electrodes (PGEs) with self-assembled CNT forests. ${ }^{37,39}$ Here, we coated disk carbonfiber microelectrodes because they have a flat surface that is easier for CNT selfassembly than cylindrical electrodes. With a high concentration of CNTs $(0.1 \mathrm{mg} / \mathrm{ml})$ and long assembly time (30 $\mathrm{min}$ ) similar to that used with PGEs, large oxidation currents (29 $\pm 7 \mathrm{nA}$ for $1 \mu \mathrm{M}$ dopamine) were observed but the response was slow and did not return to baseline (Figure 2.1). CFMEs have an area five orders of magnitude smaller than PGEs so those conditions caused a thick film to be deposited that trapped and accumulated dopamine, leading to high currents. Restricted diffusion in and out of the thick film caused the slow temporal response. 


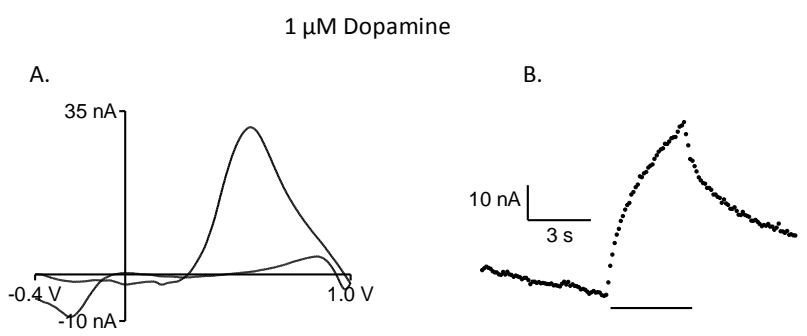

Figure 2.1 Representative cyclic voltammogram and current vs. time trace of $1 \mu \mathrm{M}$ dopamine at electrodes modified with $0.1 \mathrm{mg} / \mathrm{ml} \mathrm{CNT}$ and $30 \mathrm{~min}$ assembly time. A. The oxidation current of dopamine was large in the background subtracted cyclic voltammogram. B. The time response to a square pulse of dopamine in a flow injection analysis experiment was slow. The time when dopamine was present is marked with a line.

To optimize the response, shorter coating times and lower CNT concentrations were used. A representative cyclic voltammogram from the optimized procedure is shown in Figure 2.2A, where CNTs were assembled from a $0.02 \mathrm{mg} / \mathrm{ml}$ suspension for 5 min. The SWCNT forest electrode has a 15-fold larger background current than a bare electrode, indicative of about a 15-fold increase in electroactive surface area. The oxidation current of $1 \mu \mathrm{M}$ dopamine is approximately 30 -fold greater than the bare electrode in the background subtracted cyclic voltammograms in Figure 2.2B. Thus, the increase in oxidation current is greater than the increase in background current. The shape of the current vs time curve at the SWCNT forest electrode (Figure 2.2C) is similar to the bare electrode so the sensitivity is increased without compromising time response. 
A. Background Currents

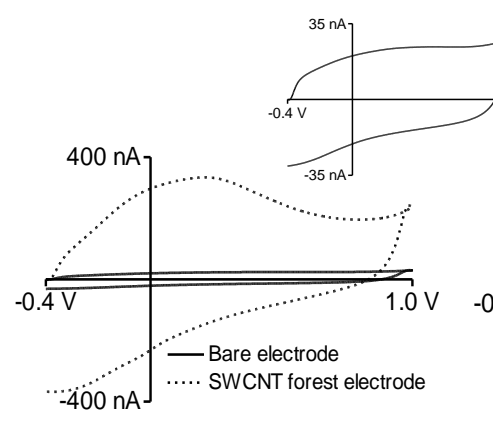

B. Dopamine Currents

C. Current vs Time Curves

Figure 2.2 Comparison of a bare (solid line) and a SWCNT forest modified (dotted line) disk carbon-fiber microelectrode for the detection of $1 \mu \mathrm{M}$ dopamine. (A) Modified electrode exhibits a 15-fold higher background current than the bare electrode. The inset is the background $\mathrm{CV}$ for the bare electrode. (B) Oxidation current of $1 \mu \mathrm{M}$ dopamine is 30-fold higher at the SWCNT forest electrode. The inset shows the dopamine CV for the bare electrode. (C) Current vs time responses for a flow injection analysis experiment. The responses were averaged from 10 electrodes each (error bars are not shown for clarity). The inset shows traces normalized to the same peak height for better comparison in peak shapes.

Carbon nanotube coating could increase electrode sensitivity by increasing electroactive surface area or adding more adsorption sites for adsorption controlled species. ${ }^{22,23}$ The ends of the CNT are proposed to have similar electrochemical activity to edge plane graphite ${ }^{30}$ and are functionalized with carboxyl groups during the shortening process. ${ }^{26}$ Here, it is highly likely that the functionalization is more responsible for the greatly improved sensitivity because electrochemical detection of dopamine is inner sphere, surface dependent, and kinetically dominated by adsorption processes at the electrode surface..$^{10,12}$ The self-assembly mechanism results in highly organized CNTs on the electrode surface and would preferentially expose the ends of the CNTs. If these ends preferentially adsorb dopamine, this would increase Faradaic current compared to the background current, which scales with area.

Table 2-1 shows the average oxidation and reduction currents for bare and SWCNT forest electrodes and the ratio of the two currents. The modified electrodes had both increased oxidation and reduction currents. The increase in the reduction current 
was larger than for the oxidation current so the ratio of the reduction to oxidation current was significantly higher at the SWCNT forest electrodes than bare electrodes $(p<0.05)$. This higher ratio indicates stronger adsorption and slower desorption kinetics of dopamine-o-quinone at the nanotubes, causing more dopamine-o-quinone to be recycled back to dopamine on the electrode surface where it may be oxidized again. This recycling may partly account for the high oxidation current at the modified electrodes. Table 2-1 also lists $\Delta \mathrm{E}_{\mathrm{p}}$ values and the $\Delta \mathrm{E}_{\mathrm{p}}$ values were not significantly different between bare and modified electrodes $(p=0.314)$. This result is consistent with a previous study that showed increased electron transfer kinetics at SWCNT dip-coated electrodes were not observed at traditional FSCV waveforms. ${ }^{22}$ The rise time from $10 \%$ to $90 \%$ of peak was calculated to quantitate the time response and the rise times were not significantly different between bare and SWCNT forest electrodes ( $p=0.404)$.

\begin{tabular}{|l|l|l|l|l|l|}
\hline & $i_{\mathrm{p}, \mathrm{a}}(\mathrm{nA})$ & $i_{\mathrm{p}, \mathrm{c}}(\mathrm{nA})$ & $i_{\mathrm{p}, \mathrm{c}} / i_{\mathrm{p}, \mathrm{a}}$ & $\Delta \mathrm{E}_{\mathrm{p}}(\mathrm{V})$ & $\begin{array}{l}\text { rise time } \\
(\mathrm{s})\end{array}$ \\
\hline Bare disk electrodes, & $0.43 \pm 0.03$ & $0.22 \pm$ & $0.49 \pm 0.03$ & $0.65 \pm$ & $0.51 \pm$ \\
$\mathrm{n}=18$ & & 0.02 & & 0.01 & 0.07 \\
\hline $\begin{array}{l}\text { SWCNT forest } \\
\text { electrodes, } \mathrm{n}=46\end{array}$ & $14 \pm 2^{\star * *}$ & $8.0 \pm$ & $0.61 \pm$ & $0.67 \pm$ & $0.58 \pm$ \\
& & $0.9^{\star \star \star}$ & $0.04^{*}$ & 0.01 & 0.04 \\
\hline
\end{tabular}

Table 2-1 Anodic and cathodic peak currents, peak separation and rise time values for 1 $\mu \mathrm{M}$ dopamine. Data are mean \pm SEM. Significant differences between bare and modified electrodes were determined by unpaired t-test $\left({ }^{* * *} p<0.001,{ }^{*} p<0.05\right)$.

For in vivo measurements, cylindrical electrodes are preferred because they have larger surface area and thus higher sensitivity. ${ }^{40}$ However, a highly sensitive disk electrode would be advantageous for spatially resolved measurements. The average oxidation current of $1 \mu \mathrm{M}$ dopamine at SWCNT forest electrodes was $14 \pm 1 \mathrm{nA}$, compared to $0.38 \pm 0.03 \mathrm{nA}$ at bare disk electrodes. The currents at SWCNT forest modified disk electrodes were higher than $30 \mu \mathrm{m}$ long, bare cylindrical electrodes which averaged $4.3 \pm 0.3 \mathrm{nA}$. To correct for differences in current due to surface area, the 
apparent geometric area of each electrode was determined from the electrode dimensions and then the current was divided by that area. The apparent current density for the SWCNT forest modified disk electrodes was $184 \pm 19 \mathrm{pA} / \mu \mathrm{m}^{2}$, larger than the 5.0 $\pm 0.4 \mathrm{pA} / \mu \mathrm{m}^{2}$ for bare disk electrodes and $6.1 \pm 0.5 \mathrm{pA} / \mu \mathrm{m}^{2}$ for bare cylindrical electrodes $(p<0.001)$. The 30 -fold larger current density is likely due to enhanced surface roughness and increased adsorption sites for dopamine.

\subsubsection{Effects of different coating methods}

To confirm the effect of the self-assembly of SWCNT forests, three control experiments were performed. First, electrodes were coated only in the dilute Nafion solution and second, by iron hydroxide-decorated Nafion. Third, bare electrodes (without Nafion/FeO $(\mathrm{OH})$ bilayer) were dipped in the same CNT-DMF suspension used for selfassembly. Figure 2.3 shows that the oxidation currents for all three control groups are not significantly different from the bare electrodes, while the SWCNT forest electrodes show a significant difference from all groups $(p<0.001)$. While Nafion is known to increase the sensitivity of electrodes, the concentration used here was 25 -fold more dilute than the typical Nafion coating procedure ${ }^{41}$ so a complete film may not form in 5 min. Elemental analysis of the electrode surface by energy dispersive X-ray spectroscopy (EDS) comparing the Nafion and Nafion/FeO $(\mathrm{OH})$ groups revealed the presence of iron hydroxide on the Nafion/FeO(OH) electrode (Figure 2.4). The small currents for electrodes dip coated with SWCNTs might be due to the low concentration of CNTs compared to previous studies. ${ }^{22,23,25}$ The large increases for the SWCNT forest group compared to the dip coated group demonstrate an effect of the self-assembly procedure, not just CNTs. 


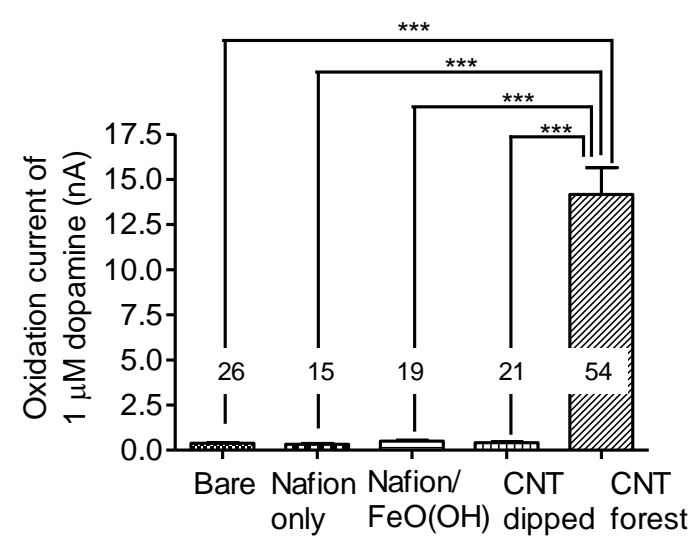

Figure 2.3 Comparison of different coatings on the oxidation current of $1 \mu \mathrm{M}$ dopamine. The Nafion only electrodes were dipped in $0.1 \%$ Nafion for $15 \mathrm{~min}$. The Nafion-FeO $(\mathrm{OH})$ electrodes were dipped sequentially in $0.1 \%$ Nafion for 15 min and $0.5 \% \mathrm{FeCl}_{3}$ for 15 min and washed with high pH DMF. For the SWCNT dip-coated group, bare electrodes were dipped in $0.02 \mathrm{mg} / \mathrm{mL}$ SWCNT DMF suspension for $5 \mathrm{~min}$. The SWCNT forest electrodes were modified with Nafion- $\mathrm{FeO}(\mathrm{OH})$ and dipped in SWCNT for $5 \mathrm{~min}$. Error bars are standard error of the mean, and the number of replicates is the number in the bars. The oxidation current of the SWCNT forest group is significantly different from all the control groups ( ${ }^{\star * *} p<0.001$, one-way ANOVA with Bonferroni post-test).

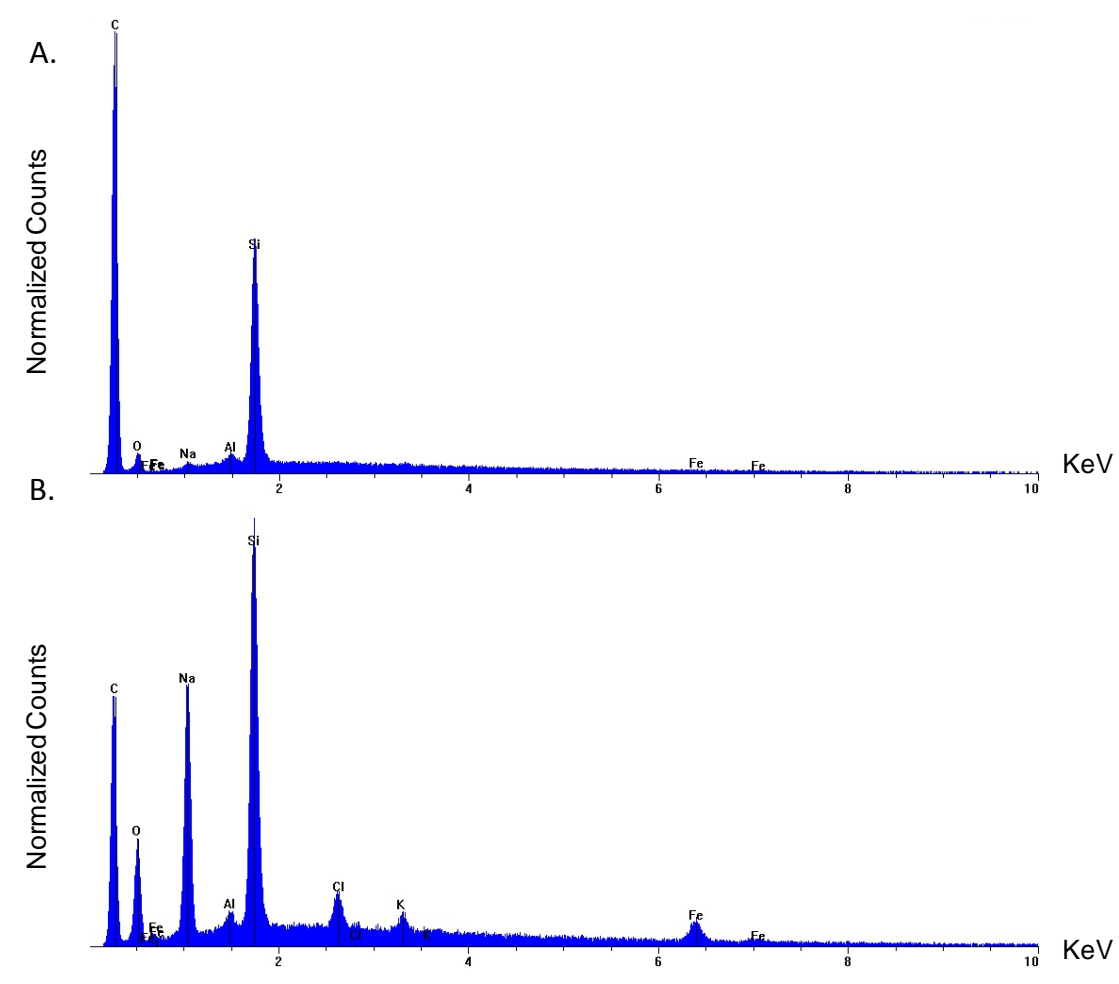

Figure 2.4 Elemental analysis of the electrode surface by energy dispersive X-ray spectroscopy (EDS) comparing A. Nafion and B. Nafion/FeO $(\mathrm{OH})$ bilayer electrode surface. The presence of the Fe peak with the increase in $\mathrm{O}$ indicated the loading of iron hydroxide. 
SEM images revealed that only random, small bundles of SWCNTs were present on the dip-coated electrode surface (Figure 2.5A). A previous study using phase contrast AFM imaging observed a more patchy appearance after SWCNT forests with 20-30 nm thickness assembled on a mica/Nafion/FeO(OH) substrate. ${ }^{42}$ Here the SEM images show that the microstructure of $\mathrm{FeO}(\mathrm{OH})$ precipitate (Figure 2.5B) were filled in by aligned CNTs after the assembly of SWCNT forests (Figure 2.5C). The electrochemistry and SEM results prove that self-assembly deposits CNTs on the electrode surface in a controllable way which facilitates a highly increased current.

A. Dip-coated SWCNTs

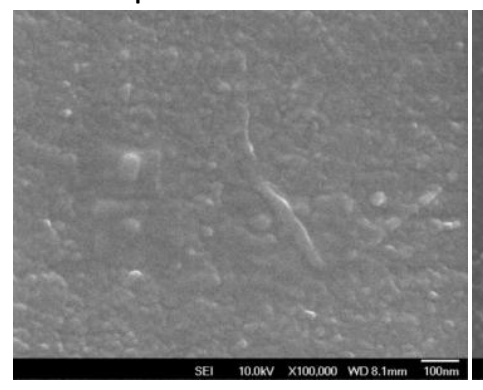

B. Nafion- $\mathrm{FeO}(\mathrm{OH})$

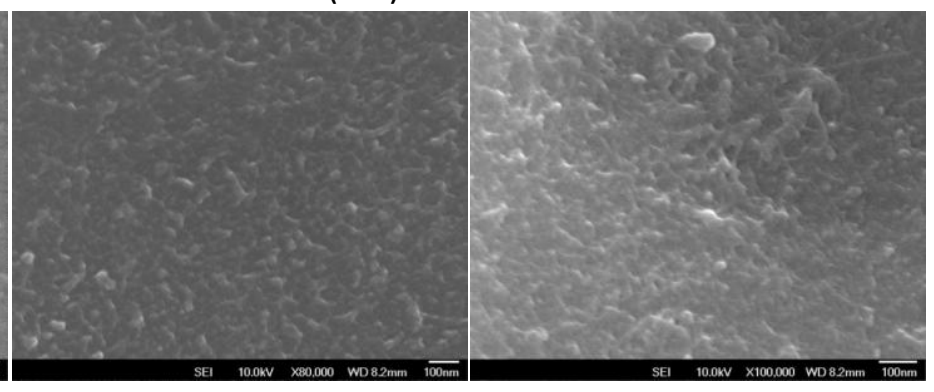

Figure 2.5 Scanning electron micrographs of electrodes. A. For the electrode dip-coated in SWCNTs, only occasional small bundles of CNTs are found on the dip-coated electrodes. B. After Nafion/ $\mathrm{FeO}(\mathrm{OH})$ coating, there is an additional microstructure. C. An image of a SWCNT forest on a Nafion/ $\mathrm{FeO}(\mathrm{OH})$ bilayer shows that structure is largely filled in with SWCNTs after self-assembly of SWCNT forest on the surface. Scale bar is $100 \mathrm{~nm}$.

The SWCNT forest electrodes also showed a significant 1.6-fold increase in S/N ratios compared to bare electrodes and a significant 1.8-fold increase compared to SWCNT dip-coated electrodes $(p<0.01)$. Dip-coated electrodes can have large agglomerations on the surface that cause large amounts of noise and decreased S/N ratios. $^{22,23,25}$ With self assembly, the CNT forest layer maximizes the accessible electroactive surface area. 


\subsubsection{Enhanced electrochemical detection for other neurochemicals}

Figure 2.6 and Table 2-2 compare the response for other electroactive neurochemicals using SWCNT forest electrodes. Norepinephrine and epinephrine are catecholamine neurotransmitters and have similar electrochemical properties to dopamine, while serotonin is an indolamine neurotransmitter with a similar oxidation potential to dopamine. ${ }^{8}$ These compounds are cationic at physiological $\mathrm{pH}$. Norepinephrine and epinephrine had similar trends as dopamine, with higher increases in oxidation current than background current. However, for serotonin, the oxidation current increase was similar to the background current increase. Serotonin has different adsorption properties than dopamine and CFMEs are highly sensitive to serotonin. ${ }^{8,43}$ Thus, the CNT ends might not preferentially adsorb serotonin as they do catecholamines. Future studies with further chemical modification of the ends of the CNT forests could test the preference for serotonin adsorbing to specific functional groups. Ascorbic acid and 3,4-dihydroxyphenylacetic acid (DOPAC) are anionic compounds, present in high concentrations in the brain, that can interfere with dopamine detection. ${ }^{3} 5$ hydroxyindoleacetic acid (5-HIAA) is an anionic metabolite of serotonin that interferes with its detection. ${ }^{8}$ The increases in oxidation current for these anionic species were smaller than that for dopamine and similar to increase in background current. Because the current for catecholamines increased more than for the anions, the SWCNT forest electrodes have better selectivity than bare electrodes. 


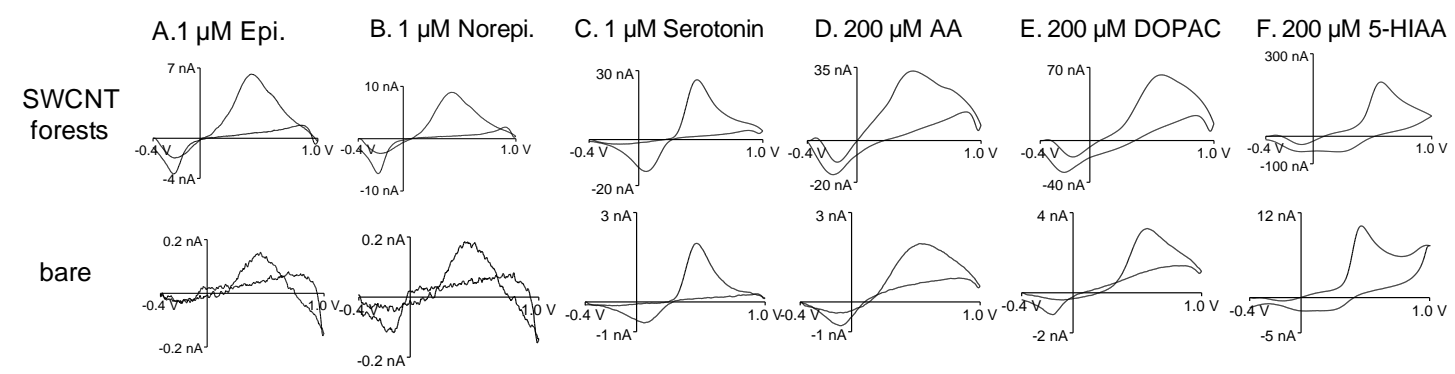

Figure 2.6 Detection of different neurochemicals at SWCNT forest electrodes. (top) CVs at SWCNT forest electrodes. (bottom) CVs at bare electrodes. The anionic species show smaller increases in oxidation current than the cationic species. Serotonin has a smaller increase than other cationic species. See Table 2-2 for average values.

\begin{tabular}{|l|l|l|l|}
\hline Analytes & $\begin{array}{l}\text { Bare } \\
\text { electrodes } \\
(n=13)\end{array}$ & $\begin{array}{l}\text { SWCNT } \\
\text { forest electrodes }(n=12)\end{array}$ & $\begin{array}{l}\text { Ratio } \\
\text { (Modified/Bare) }\end{array}$ \\
\hline Dopamine $(1 \mu \mathrm{M})$ & $0.40 \pm 0.04$ & $11 \pm 3$ & 28 \\
\hline Epinephrine $(1 \mu \mathrm{M})$ & $0.14 \pm 0.01$ & $5 \pm 1$ & 36 \\
\hline Norepinephrine $(1 \mu \mathrm{M})$ & $0.21 \pm 0.03$ & $7 \pm 1$ & 33 \\
\hline Serotonin $(1 \mu \mathrm{M})$ & $1.8 \pm 0.1$ & $25 \pm 4$ & 14 \\
\hline Ascorbic acid $(200 \mu \mathrm{M})$ & $2.0 \pm 0.1$ & $33 \pm 5$ & 17 \\
\hline DOPAC $(200 \mu \mathrm{M})$ & $3.1 \pm 0.1$ & $61 \pm 12$ & 20 \\
\hline 5-HIAA $(200 \mu \mathrm{M})$ & $10.0 \pm 0.5$ & $203 \pm 45$ & 20 \\
\hline
\end{tabular}

Table 2-2 Average oxidation currents for different neurochemicals at bare and SWCNT forest electrodes. Values are mean \pm SEM. The ratios (modified/bare) are calculated from the mean values.

\subsubsection{Improved temporal resolution at highly sensitive microelectrodes}

The high sensitivity of the SWCNT forest electrodes allows them to be used at higher repetition rates, improving temporal resolution. With traditional FSCV, the entire scan of the triangle waveform takes less than $10 \mathrm{~ms}$, but the scans are only repeated every $100 \mathrm{~ms}$ to allow time for dopamine to adsorb. ${ }^{12}$ As shown in Figure 2.7A, the oxidation current of $1 \mu \mathrm{M}$ dopamine decreases for both SWCNT forest and bare electrodes at higher repetition frequencies. However, the modified electrodes still maintain a large current at $90 \mathrm{~Hz}$, which is higher than the bare electrode at $10 \mathrm{~Hz}$ (Figure 2.7B). The increased sensitivity of the modified electrodes facilitates faster measurements and will allow more accurate determinations of the kinetics of neurotransmitter release events under physiological conditions. ${ }^{12,44}$ 
A. O Bare electrodes, $n=11$

- SWCNT forest electrodes, $n=7$

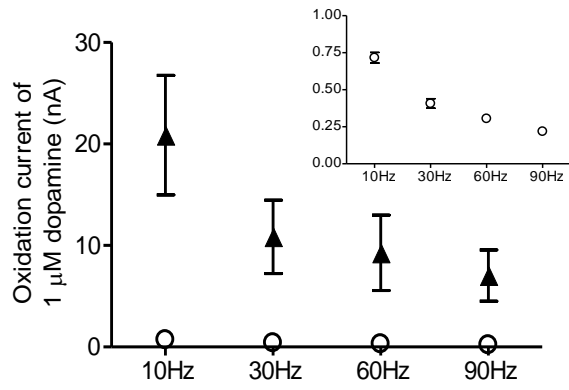

B.

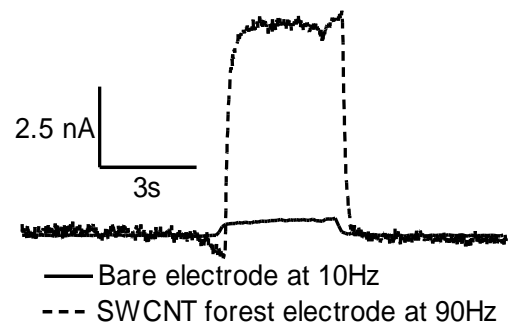

Figure 2.7 Comparison of bare and SWCNT forest modified electrodes at higher repetition frequencies. (A) Modified electrodes and bare electrodes both exhibit decreases in current at higher frequencies, but the modified electrodes still maintain a high current at $90 \mathrm{~Hz}$. The inset shows data for the bare electrodes. (B) A bare electrode at $10 \mathrm{~Hz}$ and a SWCNT forest modified electrode at $90 \mathrm{~Hz}$ for the detection of $1 \mu \mathrm{M}$ dopamine. The modified electrode has a 10-fold higher current with 9-fold better temporal resolution.

Different concentrations of dopamine were tested with bare and SWCNT forest electrodes at $10 \mathrm{~Hz}$ and $90 \mathrm{~Hz}$, respectively (Figure 2.8). At $10 \mathrm{~Hz}$, bare disk electrodes exhibited a limit of detection (LOD, $S / N=3$ ) of $63 \pm 10 \mathrm{nM}$ while the SWCNT forest electrodes had a LOD of $17 \pm 3 \mathrm{nM}$. At $90 \mathrm{~Hz}$, the LOD was $190 \pm 35 \mathrm{nM}$ for bare electrodes and $65 \pm 7 \mathrm{nM}$ for the SWCNT forest electrodes. Thus, the SWCNT forest electrodes not only exhibit a 3-fold better LOD than the bare electrodes at $10 \mathrm{~Hz}$, but notably can be used at 9-times faster repetition rate while maintaining the same LOD as traditional bare electrodes. The linear range for the SWCNT forest electrodes was comparable to the bare electrodes, with linear response up to $25 \mu \mathrm{M}$ dopamine. 
A. Modified electrodes at $10 \mathrm{~Hz}, \mathrm{n}=7$

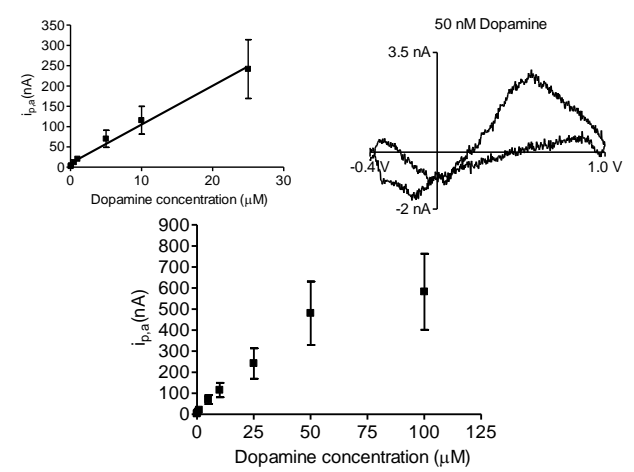

C. Bare electrodes at $10 \mathrm{~Hz}, \mathrm{n}=5$

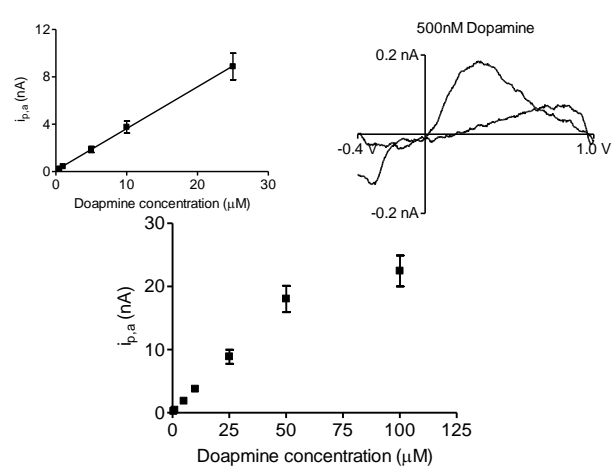

B. Modified electrodes at $90 \mathrm{~Hz}, \mathrm{n}=6$

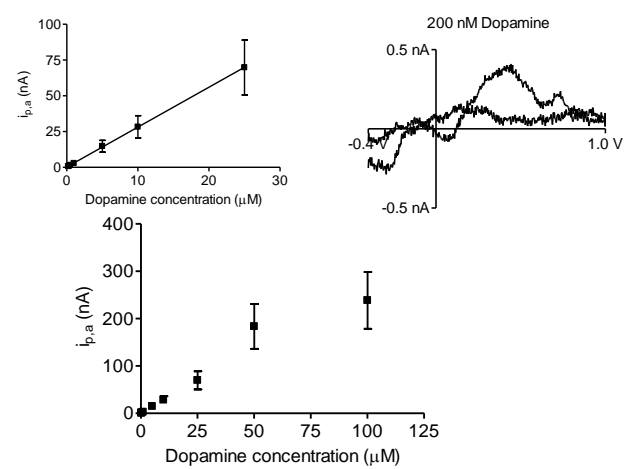

D. Bare electrodes at $90 \mathrm{~Hz}, \mathrm{n}=5$
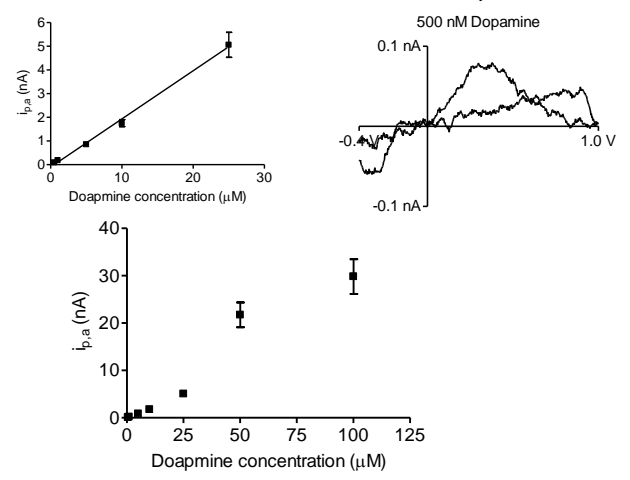

Figure 2.8 Measuring different concentrations of dopamine at $10 \mathrm{~Hz}$ and $90 \mathrm{~Hz}$ frequencies. A. SWCNT-modified electrodes were used at $10 \mathrm{~Hz}$ with concentrations from $0.05 \mu \mathrm{M}$ to $100 \mu \mathrm{M}$ dopamine. B. SWCNT-modified electrodes at $90 \mathrm{~Hz}$ repetition rates with concentrations from $0.2 \mu \mathrm{M}$ to $100 \mu \mathrm{M}$ dopamine. C. Bare electrodes at $10 \mathrm{~Hz}$ with concentration plotted from $0.5 \mu \mathrm{M}$ to $100 \mu \mathrm{M}$ dopamine. D. Bare electrodes at $90 \mathrm{~Hz}$ from $0.5 \mu \mathrm{M}$ to $100 \mu \mathrm{M}$ dopamine. Left insets show linear range is up to $25 \mu \mathrm{M}$ for all the electrodes. Right insets show the example CVs of dopamine at the lowest concentration detected.

\subsubsection{Measurements in Drosophila}

To validate that the SWCNT forest electrodes maintained the high sensitivity and improved temporal resolution in vivo, these electrodes were used to measure endogenous dopamine changes in the ventral nerve cord (VNC) of the fruit fly, Drosophila melanogaster. The flies were genetically modified to express Channelrhodopsin-2, a blue light sensitive cation channel, in only dopaminergic neurons. Dopamine release was stimulated by a pulse train ( $60 \mathrm{~Hz}, 500$ pulses) of a $473 \mathrm{~nm}$ laser. 
Figure 2.9A shows a representative color plot of stimulated dopamine release recorded at $10 \mathrm{~Hz}$ with a bare disk microelectrode. The current is small, only $0.1 \mathrm{nA}$, because the disk microelectrode has a small surface area and no current was detected when the repetition rate was increased to $90 \mathrm{~Hz}$. Figure 2.9B shows a representative color plot recorded with a SWCNT forest electrode at $90 \mathrm{~Hz}$. The currents are larger than the bare electrode at $10 \mathrm{~Hz}$. Figure $2.9 \mathrm{C}$ shows the SWCNT forest electrodes maintain significantly higher currents than the bare electrodes at both $10 \mathrm{~Hz}$ and $90 \mathrm{~Hz}$. The SWCNT forest electrodes in Drosophila also showed a slight decrease in current at higher frequencies, but the decrease was not as great as in vitro.

After use, electrodes were calibrated at the respective frequencies and then the currents in vivo were converted to concentrations. Figure 2.9D shows representative concentration traces recorded in the same sample with a SWCNT forest electrode at 10 $\mathrm{Hz}$ and $90 \mathrm{~Hz}$. The maximal evoked dopamine concentrations was $0.32 \pm 0.04 \mu \mathrm{M}$ for bare electrodes at $10 \mathrm{~Hz}, 0.42 \pm 0.07 \mu \mathrm{M}$ for SWCNT forest electrodes at $10 \mathrm{~Hz}$, and $0.46 \pm 0.11 \mu \mathrm{M}$ at $90 \mathrm{~Hz}$. As expected, these concentrations were not significantly different. The time from the start of stimulation to peak concentration and the time from peak to half decay of each trace were also compared at SWCNT forest electrodes at 10 $\mathrm{Hz}$ and $90 \mathrm{~Hz}$ and the results were not significantly different. In brain slices, Kile et al reported that dopamine evoked by a single pulse electrical stimulation rose slower and took longer to return to baseline with FSCV recorded at $10 \mathrm{~Hz}$ compared to $60 \mathrm{~Hz}{ }^{44}$ With our long stimulations, no differences in rise time or decay time were detected. Future work examining shorter stimulations might reveal more differences. However, the SWCNT forest electrodes showed potential for measuring small amounts of neurotransmitters with a fast repetition rate. 
A. Bare electrode, $10 \mathrm{~Hz}$

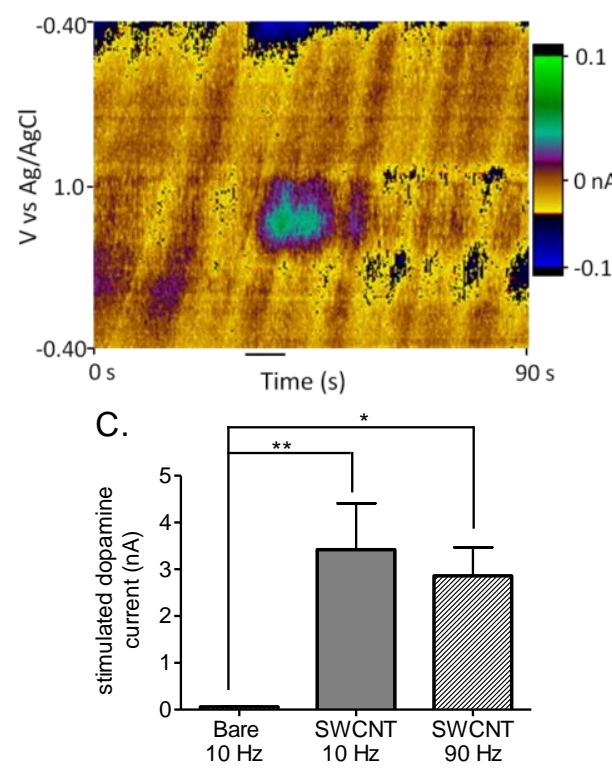

B. SWCNT forest electrode, $90 \mathrm{~Hz}$

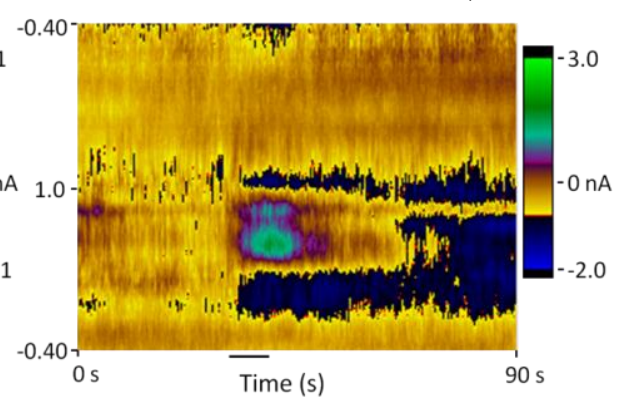

D.

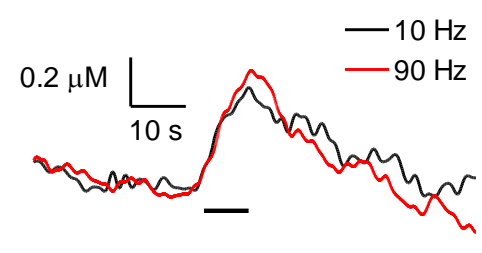

Figure 2.9 Comparison of bare and SWCNT forest modified electrodes for dopamine detection in Drosophila. A pulse train $(4 \mathrm{~ms}, 60 \mathrm{~Hz}, 500$ pulses, denoted by line under figure) of a $473 \mathrm{~nm}$ laser was applied to stimulate dopamine release (A) detected with a bare electrode at $10 \mathrm{~Hz}$ and (B) with an SWCNT forest electrode at $90 \mathrm{~Hz}$. The electrodes were placed in different samples. The color plots show all data, and dopamine oxidation is the green feature in the middle of the plot. (C) Average current detected with bare electrodes at $10 \mathrm{~Hz}$ was significantly lower than with SWCNT forest electrodes at 10 and $90 \mathrm{~Hz}\left({ }^{*} p<0.05,{ }^{* *} p<0.01,1\right.$-way ANOVA with Bonferroni posttest, $\mathrm{n}=6$ for bare and $\mathrm{n}=5$ for SWCNT forest electrodes). (D) Representative concentration traces in the same nerve cord recorded with a SWCNT forest electrode at 10 and $90 \mathrm{~Hz}$.

\subsection{Conclusions}

Highly sensitive, disk carbon-fiber microelectrodes were fabricated using a chemical self-assembly method to deposit SWCNTs on the surface. This method allowed deposition of a dense, aligned SWCNT forest on the electrode surface in a controlled fashion. Large increases in current were observed after SWCNT forests formation, likely due to the exposure of the CNT ends to the analyte. The high sensitivity of the modified electrodes facilitated the use of faster repetition rate, improving temporal resolution. The increased sensitivity as well as the improved temporal resolution were 
maintained after implantation in Drosophila, thus these modified electrodes showed great promise for studying neurotransmitter release events in biological systems.

\subsection{Methods}

\subsubsection{Solutions}

Dopamine hydrochloride (DA), epinephrine (Epi), norepinephrine (NE), serotonin hydrochloride (5-HT), ascorbic acid (AA), 3,4-Dihydroxyphenylacetic acid (DOPAC) and 5-hydroxyindoleacetic acid (5-HIAA) were purchased from Sigma-Aldrich (St. Louis, $\mathrm{MO})$. Stock solutions were made in $0.1 \mathrm{M} \mathrm{HClO}_{4}$ and were $50 \mathrm{mM}$ for $\mathrm{AA}$, DOPAC, 5HIAA and $1 \mathrm{mM}$ for DA, Epi, NE, 5-HT. Daily, solutions of $200 \mu \mathrm{M} \mathrm{AA,} \mathrm{DOPAC,} \mathrm{5-HIAA}$ or $1 \mu \mathrm{M}$ DA, Epi, NE, 5-HT were made by diluting the stock solutions in Tris buffer. The Tris buffer solution was $15 \mathrm{mM}$ Tris(hydroxymethyl)aminomethane, $3.25 \mathrm{mM} \mathrm{KCl,} 140$ $\mathrm{mM} \mathrm{NaCl}, 1.2 \mathrm{mM} \mathrm{CaCl}_{2}, 1.25 \mathrm{mM} \mathrm{NaH}_{2} \mathrm{PO}_{4}, 1.2 \mathrm{mM} \mathrm{MgCl}_{2}$ and $2.0 \mathrm{mM} \mathrm{Na}_{2} \mathrm{SO}_{4}$ with the $\mathrm{pH}$ adjusted to 7.4. A modified Schneider's buffer (15.2 $\mathrm{mM} \mathrm{MgSO}_{4}, 21 \mathrm{mM} \mathrm{KCl,} 3.3$ $\mathrm{mM} \mathrm{KH}_{2} \mathrm{PO}_{4}, 53 \mathrm{mM} \mathrm{NaCl}, 5.8 \mathrm{mM} \mathrm{NaH}_{2} \mathrm{PO}_{4}, 5.4 \mathrm{mM} \mathrm{CaCl}_{2}, 11.1 \mathrm{mM}$ glucose, $5.3 \mathrm{mM}$ trehalose, $\mathrm{pH}=6.2$ ) was used for Drosophila experiments and electrode calibrations. All chemicals were purchased from Fisher (Suwanee, GA) and solutions were made with deionized water (Milli-Q Biocel, Millipore, Billerica, MA).

\subsubsection{Electrochemistry}

Carbon-fiber microelectrodes were fabricated from T-650 carbon fibers (a gift of Cytec Engineering Materials, West Patterson, NJ). A single fiber was vacuum-aspirated into a glass capillary (1.2 mm diameter, A\&M Systems, Carlsborg, WA) and pulled on an electrode puller (Narishige, PE-21, Tokyo, Japan). The fiber was trimmed at the glass seal to make the disk electrodes. For cylindrical electrodes, the fiber was cut approximately $50 \mu \mathrm{m}$ from the glass seal. The electrodes were epoxied with Epon Resin 
828 (Miller-Stephenson, Danbury, CT) mixed with 14\% (w/w) 1,3-phenylenediamine hardener (Sigma-Aldrich, St. Louis, MO) heated to $85^{\circ} \mathrm{C}$. Electrodes were dipped in the epoxy for $30 \mathrm{~s}$. Cylindrical electrodes were dipped in acetone for another $3 \mathrm{~s}$ immediately following the epoxy step. The epoxied electrodes were cured overnight at room temperature and then heated to $100^{\circ} \mathrm{C}$ for $2 \mathrm{~h}$ and at $150{ }^{\circ} \mathrm{C}$ overnight. Disk electrodes were polished at a $30^{\circ}$ angle on a fine diamond abrasive plate (Sutter Instruments model BV-10, Novato, CA) to create an elliptical active area. The geometric surface area of the cylindrical electrode was calculated by measuring the length of the fiber under the microscope using the formula $A=2 \pi r l+\pi r^{2}$, where $r$ is the radius of the electrode and $/$ is the length. For disk electrode, the formula $A=\pi a b$ was used for an elliptical area and $a=r, b=2 r$ at $30^{\circ}$ angle polishing angle. All electrodes were soaked in isopropanol overnight before use and were back-filled with $1 \mathrm{M} \mathrm{KCl}$ to provide an electrical connection between the fiber and the wire to the headstage.

Fast-scan cyclic voltammograms were collected using a ChemClamp (Dagan, Minneapolis, MN, $\mathrm{n}=0.01$ headstage), $\mathrm{PCl} 6711$ and 6052 computer interface cards (National Instruments, Austin, TX) and a home built break-out box. Data collection was computer controlled by the TarHeel CV software program. For all neurochemical measurements, the electrode was scanned with a triangular waveform from $-0.4 \mathrm{~V}$ to $1.0 \mathrm{~V}$ vs. a $\mathrm{Ag} / \mathrm{AgCl}$ reference electrode at a scan rate of $400 \mathrm{~V} / \mathrm{s}$. The repetition rate is $10 \mathrm{~Hz}$ unless noted in the text. Electrodes were tested using flow-injection analysis as previously described ${ }^{22}$ with $4 \mathrm{~s}$ analyte injections. Cyclic voltammograms (CVs) were calculated by subtracting the average of ten background scans taken before analyte was injected from the average of five scans when analyte was present. The current $v s$. time traces were obtained by integrating the current in a $100 \mathrm{mV}$ window centered at the oxidation peak for each CV. 


\subsubsection{Electrode modification}

The functionalization of single-walled carbon nanotubes (SWCNTs) and the assembly process were adapted from Chattopadhyay et al. ${ }^{37,39}$ SWCNTs (HiPCO, Carbon Nanotechnologies, Inc. Houston, TX) were sonicated in a 3:1 mixture of $\mathrm{HNO}_{3} / \mathrm{H}_{2} \mathrm{SO}_{4}$ for 4 hours at $70{ }^{\circ} \mathrm{C}$ and then were filtered with $0.22 \mu \mathrm{m}$ pore filter membrane (Durapore Membrane; Millipore) and washed repeatedly with deionized water until $\mathrm{pH}$ was neutral. During the filtration, the short (below $0.22 \mu \mathrm{m}$ ) nanotubes passed through the membrane pores initially, but the pores were quickly blocked with longer nanotubes. The shortened CNTs were dried in vacuum overnight and then suspended in DMF by sonication for 15 hours at a concentration of $0.02 \mathrm{mg} / \mathrm{ml}$. CNT suspensions were stable for several months. TEM imaging confirmed that there were some short nanotubes, tens of nm long, in the CNT suspensions (Figure 2.10). Disk carbon-fiber microelectrodes were sequentially dipped in $0.1 \%$ Nafion (5 wt \% stock solution from Liquion-1105-MeOH, Ion Power, New Castle, DE, diluted with 9:1 v/v $\mathrm{MeOH}: \mathrm{H}_{2} \mathrm{O}$ ) for 15 minutes and freshly made aqueous $\mathrm{FeCl}_{3}(0.5 \mathrm{wt} \%, \mathrm{pH}=2.2)$ for 15 minutes. The electrodes were washed with basic DMF ( $\mathrm{pH}$ 12, adjusted with $1 \% \mathrm{NaOH})$, immersed in the CNT suspension ( $\mathrm{pH}$, adjusted with ammonium hydroxide) for 5 minutes, and washed immediately with isopropanol to remove loose nanotubes. Electrodes were dried in vacuum for at least 24 hours before use. 


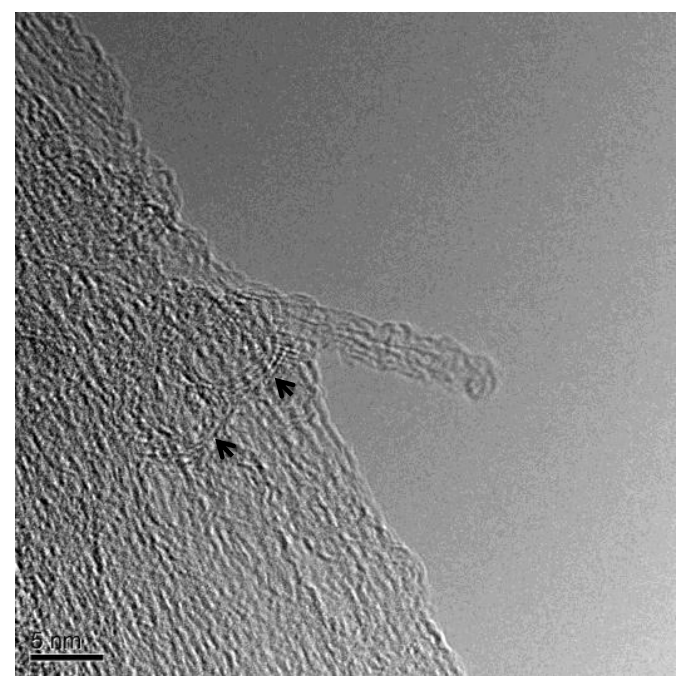

Figure 2.10 TEM image of shortened single-walled carbon nanotubes. CNT DMF suspension was spread onto a lacey carbon 200 mesh grid and then imaged. The diameter of each nanotube ranges from 0.8 to $1.2 \mathrm{~nm}$. Nanotubes tens of $\mathrm{nm}$ long are found in the sample, as indicated by arrows. Scale bar is $5 \mathrm{~nm}$.

\subsubsection{Surface structure characterization}

High resolution transmission electron microscope (TEM) investigation was carried out using a FEI Titan 80-300 system (Hillsboro, OR) with the voltage set at 300 $\mathrm{kV}$ and the CNT suspension was spread onto a lacey carbon 200 mesh grid.

Energy dispersive X-ray spectroscopy (EDS) and scanning electron microscopy (SEM) images were acquired using a JEOL JSM-6700F cold field-emission microscope (Tokyo, Japan). The EDS spectra were acquired for 300 seconds on both the Nafion and $\mathrm{Nafion} / \mathrm{FeO}(\mathrm{OH})$ bilayer electrodes with an acceleration voltage of $15 \mathrm{kV}$ at a working distance of $15 \mathrm{~mm}$. Secondary electron images were acquired with an acceleration voltage of $10 \mathrm{kV}$ at a working distance around $8 \mathrm{~mm}$. All of the samples were sputter-coated with a conductive layer of carbon ( $0.1 \AA / \mathrm{s}, 3 \mathrm{~min})$ using an precision etching coating system (PECS, 682, Gatan Inc, Pleasanton, CA). 


\subsubsection{Drosophila experiments}

Ventral nerve cord (VNC) preparation and data collection were performed as described previously. ${ }^{45}$ Homozygous 3-day-old larvae with a th-GAL4; UAS-H134RChR2 genotype were fed all-trans retinal for 2-3 days prior to the dissection and were shielded from light. The central nervous system of a wandering 3rd instar larva (5 dayold) was removed in modified Schneider's buffer and the optic lobes cut to yield an isolated VNC, which was adhered to the bottom of a plastic Petri dish containing $3 \mathrm{~mL}$ of buffer. An electrode was implanted into the VNC 4-6 segments away from the cut edge and the VNC was allowed to equilibrate for 5 minutes before data collection. 30 seconds of baseline electrochemistry data were collected before a pulse train $(4 \mathrm{~ms}, 60 \mathrm{~Hz}, 500$ pulses) of a $473 \mathrm{~nm}$ diode laser was applied (IkeCool Corporation, Los Angeles, CA). A FFT Filter smoothing (Origin-Lab OriginPro 7.5) with a cutoff frequency of $1 \mathrm{~Hz}$ was used for the in vivo data to remove noise. All experiments were performed in a dark room. Each individual electrode was tested in a separate VNC.

\subsubsection{Statistics}

All values are given as mean \pm standard error of the mean (SEM) for $n$ number of electrodes and all error bars are given as SEM. Unpaired t-tests were performed to compare properties between two groups including differences between bare and SWCNT forest electrodes and the time response of SWCNT forest electrodes at two different frequencies in Drosophila experiment. A one-way ANOVA with Bonferonni posttests was used to compare effects among multiple groups. All statistics were performed in GraphPad Prism (GraphPad Software,Inc., La Jolla, CA). 


\subsection{Reference List}

(1) Robinson, D. L.; Hermans, A.; Seipel, A. T.; Wightman, R. M. Chem. Rev. 2008, 108, 2554-2584.

(2) Kita, J. M.; Wightman, R. M. Curr. Opin. Chem. Biol. 2008, 12, 491-496.

(3) Robinson, D. L.; Venton, B. J.; Heien, M. L. A. V.; Wightman, R. M. Clin. Chem. 2003, 49, 1763-1773.

(4) Troyer, K. P.; Heien, M. L.; Venton, B. J.; Wightman, R. M. Curr. Opin. Chem. Biol. 2002, 6, 696-703.

(5) Wightman, R. M. Science 2006, 311, 1570-1574.

(6) Richfield, E. K.; Penney, J. B.; Young, A. B. Neuroscience 1989, 30, 767-777.

(7) Berke, J. D.; Hyman, S. E. Neuron 2000, 25, 515-532.

(8) Baur, J. E.; Kristensen, E. W.; May, L. J.; Wiedemann, D. J.; Wightman, R. M. Anal. Chem. 1988, 60, 1268-1272.

(9) Kawagoe, K. T.; Zimmerman, J. B.; Wightman, R. M. J. Neurosci. Meth. 1993, 48, 225-240.

(10) Bath, B. D.; Martin, H. B.; Wightman, R. M.; Anderson, M. R. Langmuir 2001, 17, 7032-7039.

(11) Duvall, S. H.; McCreery, R. L. J. Am. Chem. Soc. 2000, 122, 6759-6764.

(12) Bath, B. D.; Michael, D. J.; Trafton, B. J.; Joseph, J. D.; Runnels, P. L.; Wightman, R. M. Anal. Chem. 2000, 72, 5994-6002.

(13) Pihel, K.; Walker, Q. D.; Wightman, R. M. Anal. Chem. 1996, 68, 2084-2089.

(14) Hashemi, P.; Dankoski, E. C.; Petrovic, J.; Keithley, R. B.; Wightman, R. M. Anal. Chem. 2009, 81, 9462-9471.

(15) Heien, M. L. A. V.; Phillips, P. E. M.; Stuber, G. D.; Seipel, A. T.; Wightman, R. M. Analyst 2003, 128, 1413-1419.

(16) Takmakov, P.; Zachek, M. K.; Keithley, R. B.; Walsh, P. L.; Donley, C.; McCarty, G. S.; Wightman, R. M. Anal. Chem. 2010, 82, 2020-2028.

(17) Jacobs, C. B.; Peairs, M. J.; Venton, B. J. Anal. Chim. Acta 2010, 662, 105-127.

(18) Yang, W.; Ratinac, K. R.; Ringer, S. P.; Thordarson, P.; Gooding, J. J.; Braet, F. Angew. Chem. , Int. Ed. 2010, 49, 2114-2138.

(19) Lin, Y.; Yantasee, W.; Wang, J. Front Biosci. 2005, 10, 492-505. 
(20) Dumitrescu, I.; Unwin, P. R.; Macpherson, J. V. Chem. Commun. 2009, 68866901.

(21) Hocevar, S. B.; Wang, J.; Deo, R. P.; Musameh, M.; Ogorevc, B. Electroanalysis $2005,17,417-422$.

(22) Swamy, B. E. K.; Venton, B. J. Analyst 2007, 132, 876-884.

(23) Jacobs, C. B.; Vickrey, T. L.; Venton, B. J. Analyst 2011, 136, 3557-3565.

(24) Jeong, H.; Jeon, S. Sensors 2008, 8, 6924-6935.

(25) Peairs, M. J.; Ross, A. E.; Venton, B. J. Anal. Chem. 2011, 3, 2379-2386.

(26) Liu, J.; Rinzler, A. G.; Dai, H. J.; Hafner, J. H.; Bradley, R. K.; Boul, P. J.; Lu, A.; Iverson, T.; Shelimov, K.; Huffman, C. B.; Rodriguez-Macias, F.; Shon, Y. S.; Lee, T. R.; Colbert, D. T.; Smalley, R. E. Science 1998, 280, 1253-1256.

(27) Banks, C. E.; Compton, R. G. Analyst 2006, 131, 15-21.

(28) Banks, C. E.; Compton, R. G. Anal. Sci. 2005, 21, 1263-1268.

(29) McCreery, R. L. Chem. Rev. 2008, 108, 2646-2687.

(30) Banks, C. E.; Davies, T. J.; Wildgoose, G. G.; Compton, R. G. Chem. Commun. 2005, 829-841.

(31) Forrest, G. A.; Alexander, A. J. J. Am. Chem. Soc. 2007, 111, 10792-10798.

(32) Diao, P.; Liu, Z. Adv. Mater. 2010, 22, 1430-1449.

(33) Yan, Y. H.; Chan-Park, M. B.; Zhang, Q. Small 2007, 3, 24-42.

(34) Huang, L. M.; Jia, Z.; O'Brien, S. J. Mater. Chem. 2007, 17, 3863-3874.

(35) Gao, L. J.; Peng, A. P.; Wang, Z. Y.; Zhang, H.; Shi, Z. J.; Gu, Z. N.; Cao, G. P.; Ding, B. Z. Solid State Commun. 2008, 146, 380-383.

(36) Diao, P.; Liu, Z. F.; Wu, B.; Nan, X. L.; Zhang, J.; Wei, Z. Chemphyschem 2002, 3, 898-901.

(37) Chattopadhyay, D.; Galeska, I.; Papadimitrakopoulos, F. J. Am. Chem. Soc. 2001, 123, 9451-9452.

(38) Liu, Z. F.; Shen, Z. Y.; Zhu, T.; Hou, S. F.; Ying, L. Z.; Shi, Z. J.; Gu, Z. N. Langmuir 2000, 16, 3569-3573.

(39) Yu, X.; Chattopadhyay, D.; Galeska, I.; Papadimitrakopoulos, F.; Rusling, J. F. Electrochem. Commun. 2003, 5, 408-411.

(40) Venton, B. J.; Wightman, R. M. Anal. Chem. 2003, 75, 414A-421A. 
(41) Jones, S. R.; Garris, P. A.; Wightman, R. M. J. Pharmacol. Exp. Ther. 1995, 274, 396-403.

(42) Malhotra, R.; Papadimtrakopoulos, F.; Rusling, J. F. Langmuir 2010, 26, 1505015056.

(43) Jackson, B. P.; Dietz, S. M.; Wightman, R. M. Anal. Chem. 1995, 67, 1115-1120.

(44) Kile, B. M.; Walsh, P. L.; McElligott, Z. A.; Bucher, E. S.; Guillot, T. S.; Salahpour, A.; Caron, M. G.; Wightman, R. M. ACS Chem. Neurosci. 2012, 3, 285-292.

(45) Vickrey, T. L.; Condron, B.; Venton, B. J. Anal. Chem. 2009, 81, 9306-9313. 
Chapter 3

Optogenetic control of serotonin and dopamine release in Drosophila larvae 


\section{Chapter 3: Optogenetic control of serotonin and dopamine release in Drosophila larvae}

\section{Abstract}

Optogenetic control of neurotransmitter release is an elegant method to investigate neurobiological mechanisms with millisecond precision and cell type-specific resolution. Channelrhodopsin-2 (ChR2) can be expressed in specific neurons, and blue light used to activate those neurons. Previously, in Drosophila, neurotransmitter release and uptake have been studied after continuous optical illumination. In this study, we investigated the effects of pulsed optical stimulation trains on serotonin or dopamine release in larval ventral nerve cords. In larvae with ChR2 expressed in serotonergic neurons, low frequency stimulations produced a distinct, steady-state response while high frequency patterns were peak shaped. Evoked serotonin release increased with increasing stimulation frequency and then plateaued. The steady-state response and the frequency dependence disappeared after administering the uptake inhibitor fluoxetine, indicating that uptake plays a significant role in regulating the extracellular serotonin concentration. Pulsed stimulations were also used to evoke dopamine release in flies expressing ChR2 in dopaminergic neurons and similar frequency dependence was observed. Release due to pulsed optical stimulations was modeled to determine the uptake kinetics. For serotonin, $V_{\max }$ was $0.54 \pm 0.07 \mu \mathrm{M} / \mathrm{s}$ and $K_{\mathrm{m}}$ was $0.61 \pm 0.04 \mu \mathrm{M}$; and for dopamine, $V_{\max }$ was $0.12 \pm 0.03 \mu \mathrm{M} / \mathrm{s}$ and $K_{\mathrm{m}}$ was $0.45 \pm 0.13 \mu \mathrm{M}$. The amount of serotonin released per stimulation pulse was $4.4 \pm 1.0 \mathrm{nM}$, and the amount of dopamine was $1.6 \pm 0.3 \mathrm{nM}$. Thus, pulsed optical stimulations can be used to mimic neuronal firing patterns and will allow Drosophila to be used as a model system for studying mechanisms underlying neurotransmission. 


\subsection{Introduction}

Optogenetic control of neuronal activity is an elegant method to selectively activate neurons with widespread applications in the investigation of brain functions. Channelrhodopsin-2 (ChR2), is a blue-light activated cation channel found in $C$. reinhardtii and can be inserted into specific neurons with genetic manipulations. ${ }^{1,2}$ Upon blue light stimulation, ChR2 opens rapidly, and inward flow of cations leads to neuronal excitation. ${ }^{1,2}$ In contrast to traditional stimulation methods such as electrical or pharmacological stimulations, optical stimulation of neurons can be controlled with millisecond precision, and allows targeted activation of specific neurons in one location. In mammals, optical stimulation with ChR2 has been utilized in diverse applications such as understanding neuronal circuitry that underlies behavior and neurological disorders. ${ }^{3-6}$ Optical stimulation is especially useful for small model organisms, such as Drosophila melanogaster, the fruit fly, because the bipolar electrical stimulating electrode is large compared to the fly central nervous system (CNS). Drosophila are attractive for investigating basic neurobiological mechanisms because of their simple nervous system, analogous mechanisms of neurotransmitter release and uptake to mammals, short life cycle, and ease of genetic manipulation. ${ }^{7}$ Using cell-specific promoter elements, ChR2 can be inserted into specific neurons in Drosophila and those neurons activated by blue light illumination. ${ }^{8,9}$

Structural and functional studies of ChR2 reveal that the channel opens rapidly upon blue light stimulation to generate a large transient photocurrent, and upon continuous illumination, the photocurrent decays to a lower steady-state level, i.e. it desensitizes. ${ }^{1,2}$ When a second pulse is applied after a short dark phase, the transient current component is smaller than the first one. ${ }^{1}$ ChR2 reliably drives defined trains of spikes when the frequency of pulsed light is below $40 \mathrm{~Hz} ;{ }^{2}$ however, the correlation between light pulses and cell firing weakens above this frequency. ${ }^{10-14}$ Recent studies of 
ChR2-mediated, optically-stimulated dopamine release in vivo reveal U-shaped frequency dependence curves with $40 \mathrm{~Hz}$ evoking the maximal signal with 4-ms pulse width, indicating ChR2 kinetics may play a role in stimulated release. ${ }^{15,16}$ The pulsed, optically-stimulated dopamine release was modeled to determine the kinetics of release and uptake. ${ }^{15}$ In contrast to the pulsed optogenetic trains used in mammals, only continuous illumination stimulations have been used to measure evoked neurotransmitter release in Drosophila. ${ }^{8,9,17,18}$ Long continuous stimulations do not mimic physiological neuronal firing and the kinetics of release and uptake cannot be directly modeled.

In this study, we characterized the effect of pulsed optical stimulation trains on serotonin and dopamine release in Drosophila larval ventral nerve cords. When Drosophila serotonergic and dopaminergic neurons are selectively activated in vivo, there are large increases in the amount of firing in the $30-100 \mathrm{~Hz}$ range, as well as a smaller increase in the $2-6 \mathrm{~Hz}$ range. ${ }^{19}$ The pulsed stimulations we tested $(10-100 \mathrm{~Hz})$ mimic the faster expected firing rates. We concentrated on serotonin, as serotonin signaling plays a key role in biological processes such as mood and sleep, and the serotonin transporter is a target for many drugs designed to treat psychiatric disorders. ${ }^{20-}$ ${ }^{22}$ Release was measured using fast-scan cyclic voltammetry at a carbon-fiber microelectrode implanted in the neuropil of the fly CNS, where ChR2 has been expressed in specific neurons using the yeast GAL4/UAS system. ${ }^{8,9,23}$ Evoked serotonin release was controlled by different light parameters, including pulse frequency, pulse width and pulse number. Administration of fluoxetine, a serotonin transporter inhibitor, eliminated the frequency dependence of evoked serotonin. Pulsed stimulations were also conducted in dopaminergic neurons to evaluate the generalizability of our method, and frequency dependent release was observed. The pulsed optical stimulations allowed the release and uptake kinetics to be directly modeled. Our results demonstrate that 
pulsed optical stimulations combined with FSCV detection can be used to study the effect of different neuronal firing patterns on uptake and release, strengthening the utility of Drosophila as a model system for studying mechanisms underlying neurotransmission.

\subsection{Results and Discussion}

\subsubsection{The shape of optically-stimulated serotonin release is frequency dependent}

Enodgenous serotonin had previously been detected in Drosophila larval ventral nerve cords (VNC) using continuous, blue light stimulation. Larvae expressing ChR2 only in serotonergic neurons, which express tryptophan hydroxylase, were used to ensure stimulation specificity. ${ }^{8,17}$ With continuous stimulation, the peak concentration increases as a function of stimulation duration until saturation is reached at $10 \mathrm{~s} .{ }^{8}$ With stimulations longer than $10 \mathrm{~s}$, the peak concentration no longer increases but plateaus, due to depletion of the releasable serotonin pool and a balance between release and uptake.

To compare pulsed and continuous stimulations, a variety of stimulations were performed with $2 \mathrm{~s}$ of light illumination. Figure 3.1 shows serotonin release in the same larval CNS evoked by $2 \mathrm{~s}$ continuous illumination, a $4 \mathrm{~ms}$ pulse width stimulation at a low frequency $(20 \mathrm{~Hz})$, and a $4 \mathrm{~ms}$ pulse width stimulation at a high frequency $(100 \mathrm{~Hz})$. Continuous illumination with $2 \mathrm{~s}$ of light produces a peak-shaped response (Figure 3.1A). With the low stimulation frequency, the concentration of serotonin increases rapidly upon stimulation but then reaches a steady-state level (Figure 3.1B). In contrast, with the high frequency train, the serotonin concentration increases during the entire stimulation to a maximum and then falls to baseline after stimulation stops (Figure 3.1C), similar to the continuous stimulation (Figure 3.1A). The maximum concentration evoked by the high 
frequency train is much higher than by the low frequency, but is less than that evoked by the $2 \mathrm{~s}$ continuous illumination.

(A) $2 \mathrm{~s}$ continuous light

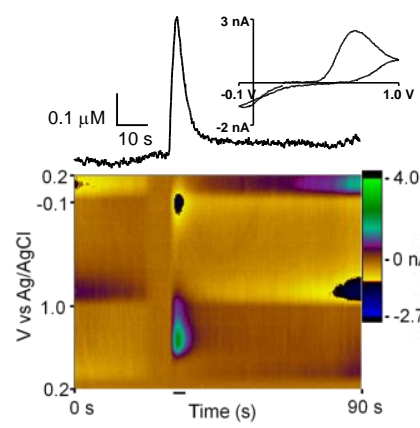

(B) $4 \mathrm{~ms}, 500$ pulses, $20 \mathrm{~Hz}$

(C) $4 \mathrm{~ms}, 500$ pulses, $100 \mathrm{~Hz}$

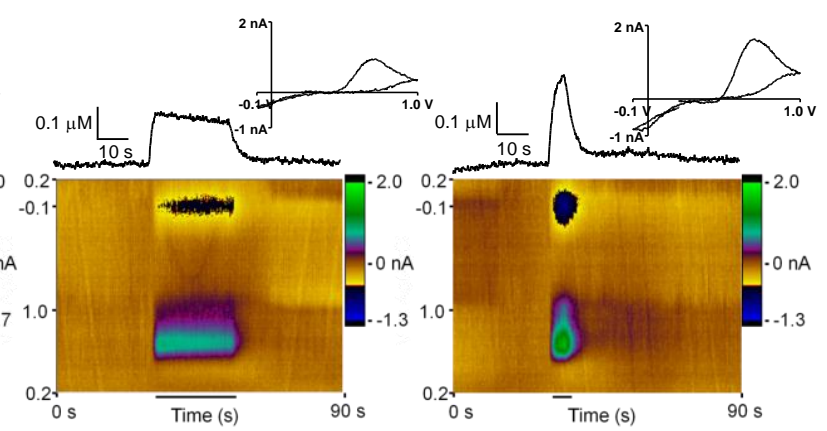

Figure 3.1 Representative serotonin release evoked by (A) 2 s continuous stimulation, (B) a pulsed stimulation train of $4 \mathrm{~ms}$ pulse width, 500 pulses at $20 \mathrm{~Hz}$, and (C) a pulsed stimulation train of $4 \mathrm{~ms}$ pulse width, 500 pulses at $100 \mathrm{~Hz}$ in the same ventral nerve cord. The bottom panel shows false color plots with time on the $\mathrm{x}$-axis, applied voltage on the $y$-axis and background-subtracted faradaic current in pseudocolor. The duration of the stimulation is marked as the black bar below the color plot. The concentration versus time profiles are plotted on top of the color plots by converting the current at the maximal oxidation potential for serotonin to concentration through in vitro calibration. The insets are background-subtracted cyclic voltammograms which confirm that serotonin is detected.

\subsubsection{The amount of serotonin released is frequency dependent}

The effect of different stimulation train parameters on evoked serotonin release was evaluated. First, the effect of stimulation frequency was tested with three pulse widths: 4, 10 and $20 \mathrm{~ms}$, a range which has been used for study of optical stimulation in rats. ${ }^{15}$ This experiment was performed in two patterns, both commonly used in investigating the frequency effect in mammals. ${ }^{15,24-26}$ In the first pattern, the total amount of time that the light was illuminated was fixed at $2 \mathrm{~s}$, that is, the product of pulse width and pulse number was kept constant at $2 \mathrm{~s}$ (Figure 3.2 A-C). For pulsed stimulations, this means that the total stimulation duration was greater than $2 \mathrm{~s}$. In the second pattern, the total duration of the stimulation was fixed at $2 \mathrm{~s}$; thus, the pulse number was adjusted to keep the product of frequency and pulse number constant at $2 \mathrm{~s}$ (Figure 3.2 D-F). Evoked release varies for different samples, so for each fly CNS, serotonin release 
was first evoked with $2 \mathrm{~s}$ continuous stimulation and then the peak concentration for each pulse train was normalized to that of the $2 \mathrm{~s}$ continuous stimulation. This ratio is plotted in Figure 3.2.

The peak concentrations evoked by pulsed stimulation trains were all lower than that evoked by the $2 \mathrm{~s}$ continuous illumination (all the ratios in Figure 3.2 are below 1 ). While continuous stimulation produced the highest serotonin concentration, the pattern and timing of action potentials fire are not known. ChR2 can drive irregular spikes under continuous illumination ${ }^{1}$, and the high concentration evoked by the continuous light is likely due to an increased probability of ChR2-driven spikes in a short amount of time, as well as less time allowed for uptake.

For pulsed stimulations, a clear frequency dependence is observed in all the panels of Figure 3.2 and the general trend is that more serotonin is evoked with higher frequency. There was a significant effect of stimulation frequency on the amount of serotonin released for each graph (one-way ANOVA, all $p<0.0001$, see figure legend for F values). In the first pattern (Figure 3.2 A-C), the release plateaued at higher frequencies. Bonferroni post-tests showed no significant differences in release for any points over $40 \mathrm{~Hz}$ for $4 \mathrm{~ms}$ (Figure $3.2 \mathrm{~A}$ ) and $10 \mathrm{~ms}$ (Figure $3.2 \mathrm{~B}$ ) duration stimulations and over $20 \mathrm{~Hz}$ for $20 \mathrm{~ms}$ (Figure $3.2 \mathrm{C}$ ) stimulations ( $>0.05)$. The peak concentrations were lower in the second pattern compared to the first pattern (Figure 3.2 D-F), due to shorter duration of the applied stimulation train. Bonferroni post-tests in one way ANOVA analysis showed release plateaued over $80 \mathrm{~Hz}$ for $4 \mathrm{~ms}$ (Figure $3.2 \mathrm{D}$ ) and $60 \mathrm{~Hz}$ for 10 ms (Figure 3.2 E), with no plateau observed for $20 \mathrm{~ms}$ (Figure 3.2 F). Below the plateau frequency, release increased linearly with stimulation frequency (Figure 3.2 D-E, linear regression, $\left.R^{2}>0.95\right)$. 

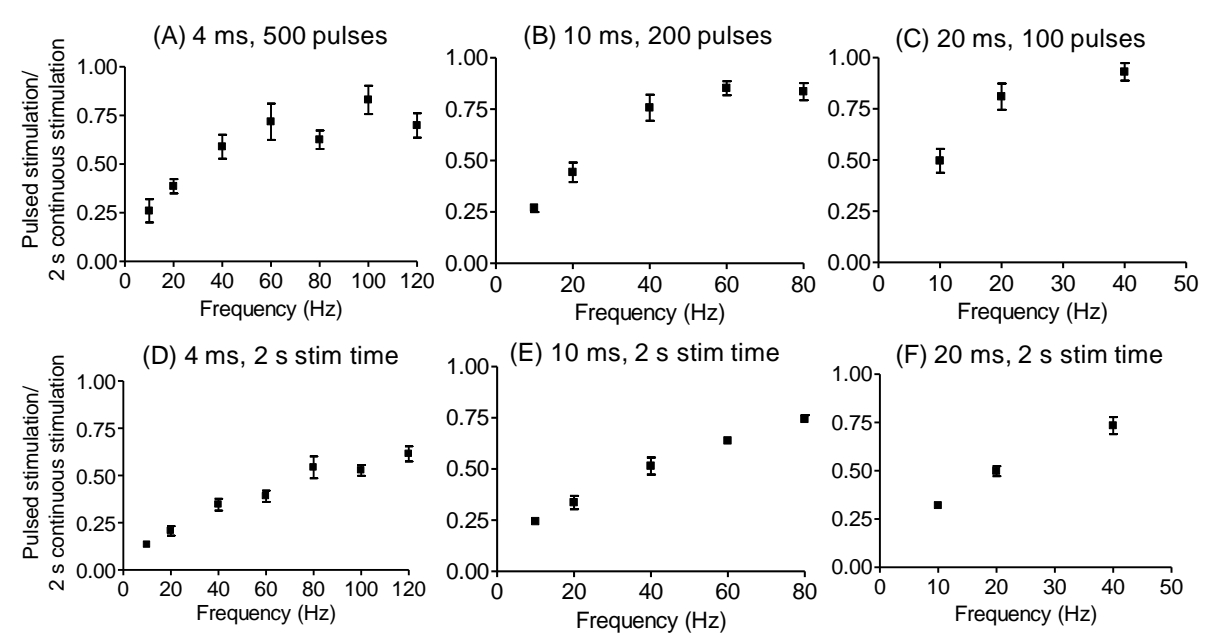

Figure 3.2 Frequency dependency of stimulated serotonin release for three different pulse widths: 4,10 , and $20 \mathrm{~ms}$. The frequency dependency is tested in two patterns. $(A-C)$ The total amount of light illumination is fixed at $2 \mathrm{~s}$. (D-F) The total simulation duration is fixed at $2 \mathrm{~s}$. It is $20,40,80,120,160,200$, and 240 pulses for $10,20,40,60$, 80,100 , and $120 \mathrm{~Hz}$, respectively. Data are expressed as the ratio of serotonin release by pulsed stimulation to that released by $2 \mathrm{~s}$ continuous illumination, which normalizes for different release amounts in different samples. Each panel was evaluated with a oneway ANOVA: (A) $F[6,41]=11.27, p<0.0001$; (B) $F[4,27]=35.46, p<0.0001$; (C) $F[2,15]$ $=16.14, p<0.001 ;(D) F[6,50]=24.89, p<0.0001 ;(E) F[4,34]=55.29, p<0.0001 ;(F)$ $F[2,21]=44.35, p<0.0001$. Data are mean \pm SEM, and $n=5-9$.

Next, the effect of pulse width was investigated by using three different frequencies and fixing the total stimulation time at $2 \mathrm{~s}$. All data are normalized to that of $2 \mathrm{~s}$ continuous illumination. Evoked serotonin increased as the pulse width increased (Figure 3.3). A one way ANOVA revealed a significant main effect of pulse width on the amount of serotonin released at all the selected frequencies $(p<0.0001)$. The $40 \mathrm{~ms}$ pulse width could not be used with $40 \mathrm{~Hz}$ stimulation because the width of the pulse is greater than the period between pulses. At all the selected frequencies, release increased linearly with pulse width (Figure 3.3 , linear regression, $R^{2}>0.98$ ). 
(A) $10 \mathrm{~Hz}, 20$ pulses

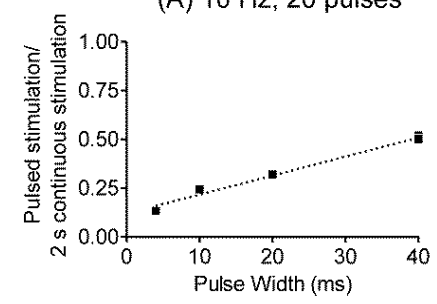

(B) $20 \mathrm{~Hz}, 40$ pulses

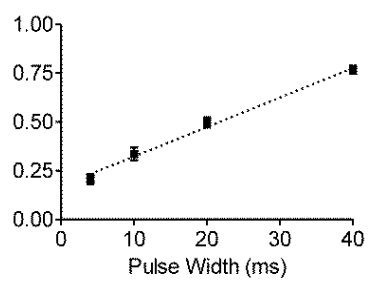

(C) $40 \mathrm{~Hz}, 80$ pulses

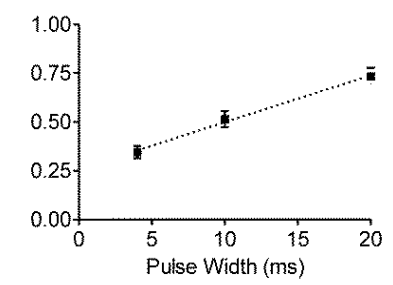

Figure 3.3 Pulse width dependency of stimulated serotonin release with three different stimulation frequencies: (A) $10 \mathrm{~Hz}$ (one-way ANOVA, $F[3,27]=55.34, p<0.0001$ ), (B) $20 \mathrm{~Hz}(\mathrm{~F}[3,28]=77.24, \mathrm{p}<0.0001)$, and $(\mathrm{C}) 40 \mathrm{~Hz}(\mathrm{~F}[2,21]=24.49, \mathrm{p}<0.0001)$. The total stimulation duration is $2 \mathrm{~s}$. Data are expressed as the ratio of serotonin release by pulsed stimulation to that of the $2 \mathrm{~s}$ continuous illumination. Data are mean \pm SEM, and $\mathrm{n}=7-8$.

Stimulated serotonin and dopamine release in mammals follows similar patterns to those observed for Drosophila larvae. A steady-state response is seen at low frequencies and release is frequency dependent. ${ }^{27,28}$ The frequency dependence varies with uptake rate ${ }^{26}$ and frequency independent evoked serotonin is found in areas with low uptake rates and in both serotonin transporter knockout and overexpressing mouse brain slices. ${ }^{24,29}$ Pulse width dependence has also been reported for electrically stimulated serotonin and dopamine release in anesthetized rats, with greater release observed for wider stimulus pulses. ${ }^{30}$

The frequency dependence of serotonin release might also depend on the ChR2 photocycle kinetics. During blue light stimulation, the photocurrent of ChR2 increases rapidly and transiently and then decays to a lower steady-state level. ${ }^{1,2}$ The efficiency of optical stimulation at higher stimulation rates is limited by ChR2 photocycle kinetics ${ }^{31-33}$, but can also be affected by its distribution, the tissue properties, and the characteristics of the light pulse. ${ }^{34}$ Computations and experiments in the hippocampus have confirmed that higher light irradiance levels, longer light pulses, and increased channel density could evoke action potentials with greater probability. ${ }^{2,34}$ In our experiments in Drosophila larval VNCs, stimulated serotonin release increased with longer pulse width and with higher light intensity (Figure 3.8), consistent with those electrophysiological 
studies $^{2,34}$ as well as studies of optogenetic control of dopamine release in anesthetized rats and rat brain slices..$^{15,16}$

Electrophysiological studies with ChR2-expressing cells have demonstrated that many cells fail to follow the ChR2-driven spikes above the $40 \mathrm{~Hz}$ range in sustained trains ${ }^{2,10,11}$ due to slow recovery from inactivation of $\mathrm{ChR} 2^{31-33}$ or host cell-specific properties of potassium and sodium channel activation/inactivation kinetics..$^{35,36}$ In anesthetized rats, the optically stimulated dopamine response peaked at $40 \mathrm{~Hz}$ with 4ms pulse width and then decreased, suggesting that ChR2 photocycle kinetics might limit stimulated release.${ }^{15}$ However, in Drosophila, with short stimulation pulses, the serotonin concentration is linear with frequency up to $80 \mathrm{~Hz}$, indicating that release may not be as dependent on ChR2 kinetics.

\subsubsection{The amount of serotonin release is dependent on the number of stimulation pulses}

The effect of pulse number on stimulated serotonin release was tested with a low stimulation frequency $(20 \mathrm{~Hz}$, Figure $3.4 \mathrm{~A}$ and C) and a high frequency $(60 \mathrm{~Hz}$, Figure 3.4 $\mathrm{B}$ and D). All stimulations have a $4 \mathrm{~ms}$ pulse width and all data are normalized to that of $2 \mathrm{~s}$ continuous illumination. Figure $3.4 \mathrm{~A}-\mathrm{B}$ show evoked serotonin increases with larger pulse numbers and then plateaus. One way ANOVA analysis showed a significant overall effect of pulse number on the amount of serotonin released for both frequencies $(F[8,45]=3.01, p<0.01$ for $20 \mathrm{~Hz}$ and $F[13,56]=19.18, p<0.0001$ for $60 \mathrm{~Hz})$. The lower frequency stimulation plateaus at a lower number of pulses than the higher frequency stimulation ( 40 pulses for $20 \mathrm{~Hz}$ vs 240 pulses for $60 \mathrm{~Hz}$ ). This is illustrated in Figure 3.4 C and D, where the $60 \mathrm{~Hz}$ stimulations were peak shaped for 120 pulses but steady-state profile for 480 pulses, while the $20 \mathrm{~Hz}$ stimulations had a steady-state profile by 100 pulses. The steady-state serotonin level for $60 \mathrm{~Hz}$ stimulations is much 
higher than that of $20 \mathrm{~Hz}$ stimulations (Figure $3.4 \mathrm{C}$ and D). Notably, even for the high stimulation frequency $(60 \mathrm{~Hz})$, the plateau concentration was much lower than plateau concentration of long duration continuous stimulations, ${ }^{8}$ indicating the plateau concentration at large pulse numbers is a balance between release and uptake, not due to depletion of the releasable serotonin pool.

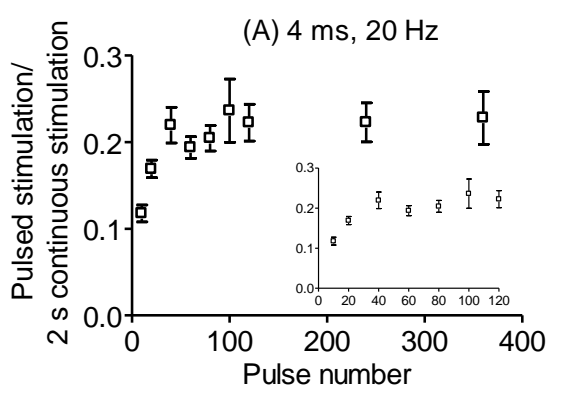

(C)

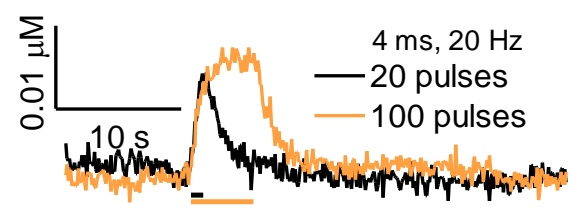

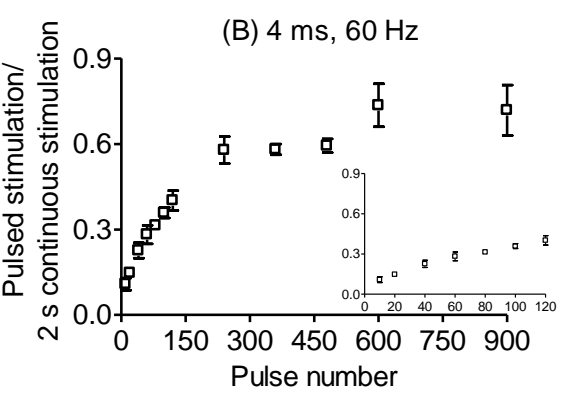

(D)

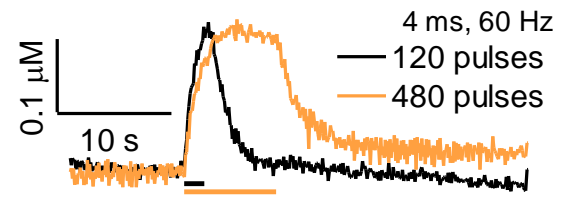

Figure 3.4 Effect of pulse number on stimulated serotonin release. The effect is tested at two different frequencies (A) $20 \mathrm{~Hz}$ and (B) $60 \mathrm{~Hz}$ with a 4 ms pulse width. Data are expressed as the ratio between serotonin release by pulsed stimulation and that of the 2 $s$ continuous illumination. Data are mean $\pm \operatorname{SEM},(A) n=4-7$ and $(B) n=6$. Insets are enlarged view of the first 120 pulses. (C) Data from one representative nerve cord at 4 ms, $20 \mathrm{~Hz}$ stimulation with two pulse numbers (20 pulses and 100 pulses). A steadystate is achieved with the higher pulse number while not with the lower one. (D) Similarly, data recorded from one nerve cord with $4 \mathrm{~ms}, 60 \mathrm{~Hz}$ stimulation using two pulse numbers (120 pulses and 480 pulses). The duration of the stimulation is marked below the concentration traces (black solid bar for the shorter stimulation and orange solid bar for the longer stimulation). The y scale in (D) is 10 times larger than the y scale in (C).

\subsubsection{Serotonin release and uptake kinetics following pulsed optical stimulation} trains

The most attractive application of pulsed optical stimulations in Drosophila is modeling of neurotransmitter release and uptake kinetic parameters directly from individual response curves. The extracellular neurotransmitter concentrations were 
modeled as a balance between release and uptake. According to the commonly used model of pulsed electrical stimulation in mammals, release is a discontinuous process whereby each stimulus pulse results in a discrete release of neurotransmitter causing an instantaneous increase in extracellular concentration, and uptake is treated as a continuous process that follows Michaelis-Menten kinetics. ${ }^{27,28,37,38}$ This model is simplified, particularly because it assumes that the release per pulse is constant. Frequency dependent modulation by autoreceptors might result in different amounts released per pulse. ${ }^{26,27}$ The changes in extracellular neurotransmitter levels during and after stimulation were fit using the equation:

$d[A] / d \mathrm{t}=f^{*}[A]_{\mathrm{p}}-V_{\max } /\left(K_{\mathrm{m}} /[A]+1\right)$

where $f$ is the stimulation frequency $(\mathrm{Hz}),[A]_{\mathrm{p}}$ is the concentration of neurotransmitter release per stimulus pulse, $V_{\max }$ is the maximal rate of uptake, which is dependent on the number of related transporters, and $K_{\mathrm{m}}$ is the transporter affinity. We used this simplified model to extract basic parameter estimates by fitting the raw data from Drosophila larval VNCs.

Figure 3.5 shows representative serotonin responses evoked by pulsed stimulation trains with a steady state concentration (4 ms, $20 \mathrm{~Hz}$ ) and a non-steady state concentration $(4 \mathrm{~ms}, 60 \mathrm{~Hz}$ ) from the same sample, fit with the Michaelis-Menten model. Both curves were fit with the same set of parameters. The average [Serotonin] $]_{p}$ was 4.4 $\pm 1.0 \mathrm{nM}, V_{\max }$ was $0.54 \pm 0.07 \mu \mathrm{M} / \mathrm{s}$ and $K_{\mathrm{m}}$ was $0.61 \pm 0.04 \mu \mathrm{M}$ (35 curves from 28 animals). 
(B)

$4 \mathrm{~ms}, 60 \mathrm{~Hz}, 250$ pulses

(A)

$4 \mathrm{~ms}, 20 \mathrm{~Hz}, 250$ pulses
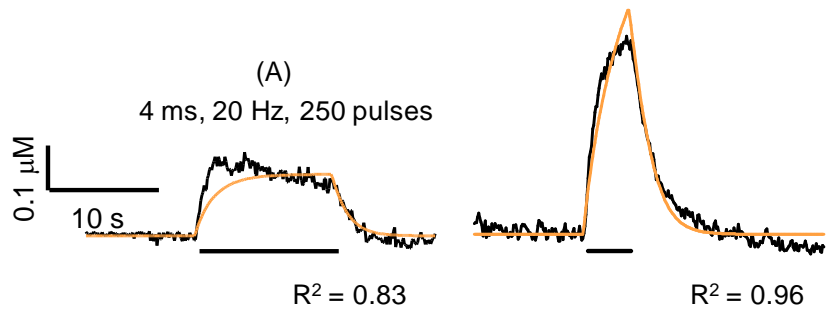

Figure 3.5 Kinetic modeling of pulsed optically stimulated serotonin release. Data from one representative nerve cord (black lines) with two different stimulation frequencies were fit to a Michaelis-Menten kinetic model to determine the parameters for serotonin release and uptake. Curves with steady-state $(A)$ and non-steady-state $(B)$ were selected for the kinetic modeling. Scale bar is the same for both panels. Simulation lines (orange) were calculated from best-fit parameters ([serotonin $]_{p}=4.3 \mathrm{nM}, V_{\max }=0.48$ $\mu \mathrm{M} / \mathrm{s}$ and $\left.K_{\mathrm{m}}=0.61 \mu \mathrm{M}\right)$. The duration of the stimulation is indicated by the black bar under the curves.

\subsubsection{The effects of uptake inhibition on optically stimulated serotonin response}

To evaluate the effects of uptake on optically stimulated serotonin response, we blocked serotonin transporter function with $100 \mu \mathrm{M}$ fluoxetine. Fluoxetine is a selective serotonin reuptake inhibitor in humans. Although it has a lower affinity and selectivity in Drosophila compared to mammalian systems, ${ }^{39,40}$ fluoxetine has also been used to effectively inhibit serotonin reuptake in Drosophila. ${ }^{8,17}$ The ability of fluoxetine to block uptake in the larval VNC was confirmed by testing $2 \mathrm{~s}$ continuous stimulation. An initial 2 s continuous stimulation was performed, then the VNC bathed in $100 \mu \mathrm{M}$ fluoxetine for 15 min and the same VNC stimulated again. The half decay time $\left(t_{50}\right)$ significantly increased after fluoxetine ( $n=15$, paired t test, $p<0.001$, Figure 3.6 A). Thus, $100 \mu \mathrm{M}$ fluoxetine effectively blocks serotonin uptake and was used to investigate the effect of uptake inhibition on pulsed stimulations.

Figure 3.6B shows serotonin release in the same VNC evoked by low frequency $(20 \mathrm{~Hz})$ and high frequency $(100 \mathrm{~Hz})$ stimulations before and after fluoxetine. After fluoxetine, the time response for both frequencies was slowed and the steady-state 
response at the low frequency became peak-shaped with a larger peak concentration. The low and high stimulation frequencies produced similar serotonin peak concentrations after fluoxetine; thus uptake regulates concentration and causes the steady-state response for low frequency stimulations. The frequency dependence of evoked serotonin release was investigated in the presence of uptake inhibitor with $4 \mathrm{~ms}$ pulses and the total amount of light illumination fixed at $2 \mathrm{~s}$ (Figure $3.6 \mathrm{C}$ ). There was no significant effect of stimulation frequency on the amount of serotonin released $(F[4,45]=$ 1.403, $p=0.2483$, one way ANOVA), in contrast to the frequency dependence observed in the low frequency range without an uptake inhibitor (Figure 3.2A). Our results in Drosophila larvae are consistent with results in mouse brain slices where electrically evoked serotonin transients were not frequency dependent in serotonin transporter knockout mice or in wild type mice after uptake inhibition. ${ }^{29}$ Furthermore, with the uptake blocker, there was no significant difference on the peak concentration of evoked serotonin release among different stimulation frequencies, indicating ChR2 kinetics was not a limiting factor in our study.

Finally, serotonin release and uptake kinetics were evaluated in the presence of an uptake inhibitor. As a competitive uptake inhibitor, fluoxetine should increase the apparent value of $K_{\mathrm{m}}$ with little effect on release and $V_{\max }{ }^{27}$ Thus, we fixed [serotonin] and $V_{\max }$ at the predrug values and floated $K_{\mathrm{m}}$. Figure 3.6D shows a representative serotonin response in the presence of $100 \mu \mathrm{M}$ fluoxetine fit with the Michaelis-Menten model. The average of $K_{\mathrm{m}}$ after fluoxetine was $5.8 \pm 0.4 \mu \mathrm{M}$ (20 curves from 10 animals), a 9-fold increase. This increase is of similar magnitude to those observed in the substantia nigra reticulata and the dorsal raphe of rat brain slices after application of fluoxetine. ${ }^{27}$ 
(A)

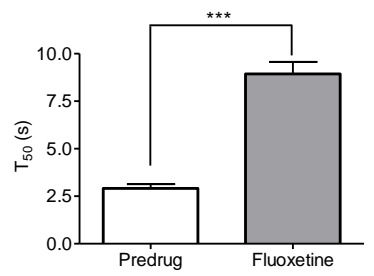

(B)
(C)

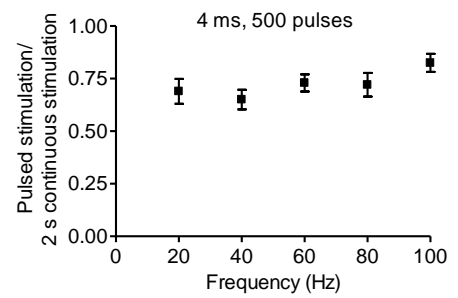

(D)

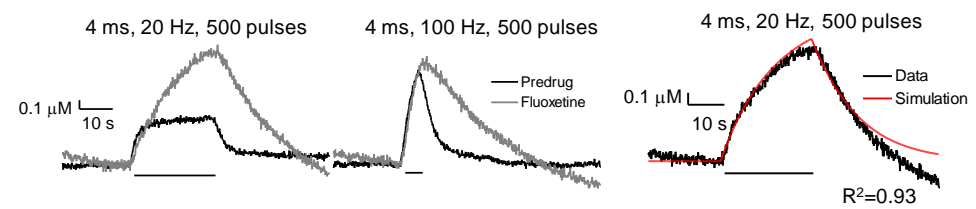

Figure 3.6 Effects of serotonin uptake inhibitor fluoxetine. (A) With $2 \mathrm{~s}$ continuous stimulation, the half decay time $\left(t_{50}\right)$ significantly increased 15 min after $100 \mu \mathrm{M}$ fluoxetine was applied $\left({ }^{* * *} p<0.001\right.$, paired $t$ test, $\left.n=15\right)$. (B) Concentrations versus time profiles showing the effect of $100 \mu \mathrm{M}$ fluoxetine on serotonin release by $4 \mathrm{~ms}$ pulse width stimulations at a low $(20 \mathrm{~Hz})$ and a high $(100 \mathrm{~Hz})$ frequency in the same nerve cord (black bar marks the stimulation duration). (C) Serotonin release is not frequency dependent after $100 \mu \mathrm{M}$ fluoxetine (data mean \pm SEM and $n=9-11$ ). Data are expressed as the ratio of serotonin release by pulsed stimulation to that released by $2 \mathrm{~s}$ continuous illumination in the presence of fluoxetine. (D) Kinetic modeling of pulsed optically stimulated serotonin release in the presence of $100 \mu \mathrm{M}$ fluoxetine. Simulation line (orange) to fit the representative data (black) is calculated with the parameters: [serotonin $]_{\mathrm{p}}=4.4 \mathrm{nM}, V_{\max }=0.54 \mu \mathrm{M} / \mathrm{s}$, and $K_{\mathrm{m}}=6.4 \mu \mathrm{M}$, with $\mathrm{R}^{2}=0.93$.

\subsubsection{Dopamine release and uptake kinetics following pulsed optical stimulation}

trains

To evaluate the generality of the parameters for optically-induced release, pulsed stimulations were also conducted in Drosophila larval VNC expressing ChR2 in neurons containing tyrosine hydroxylase, a dopaminergic synthesis enzyme. Figure 3.7A shows the frequency dependence of $4 \mathrm{~ms}$ pulse width train evoked dopamine release with the total stimulation duration fixed at $2 \mathrm{~s}$. One-way ANOVA analysis showed a significant effect of stimulation frequency $(F[6,21]=15.21, p<0.0001)$. The frequency response of dopamine is similar to that of serotonin (Figure 3.2D) and the release plateaued after 60 $\mathrm{Hz}$ (any two frequencies over $60 \mathrm{~Hz}$ are not significantly different, Bonferroni post-tests in one way ANOVA, $p>0.05)$. Figure 3.7C shows a representative dopamine response fit 
well with the kinetic model. The average [dopamine] $]_{p}$ was $1.6 \pm 0.3 \mathrm{nM}, V_{\max }$ was $0.12 \pm$ $0.03 \mu \mathrm{M} / \mathrm{s}$, and $K_{\mathrm{m}}$ was $0.45 \pm 0.13 \mu \mathrm{M}$ (11 curves from 5 animals).

(A)

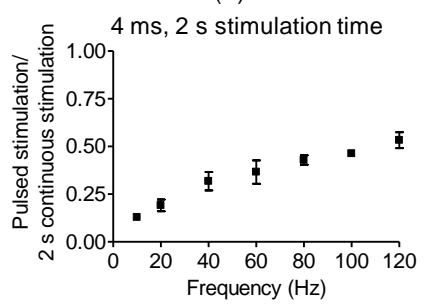

(C)

$4 \mathrm{~ms}, 40 \mathrm{~Hz}, 80$ pulses

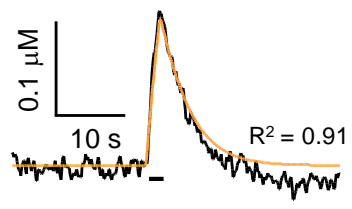

(B)

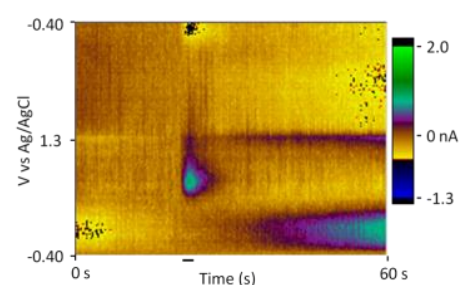

(D)

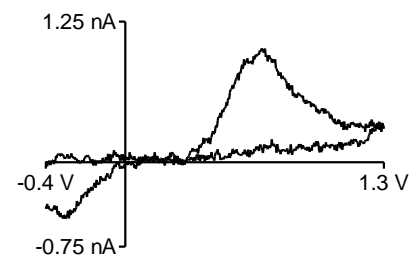

Figure 3.7 Dopamine release evoked by pulsed optical stimulations. (A) Frequency dependence of stimulated dopamine release with $4 \mathrm{~ms}$ pulse width and the total stimulation duration is fixed at $2 \mathrm{~s}$. Data are expressed as the ratio of dopamine release by pulsed stimulation to that of the $2 \mathrm{~s}$ continuous illumination. Data are mean $\pm S E M, n$ $=4$. (B) Representative color plot of dopamine release evoked by a pulsed stimulation train of $4 \mathrm{~ms}, 40 \mathrm{~Hz}, 80$ pulses. The green and blue areas show the oxidation and reduction peaks of dopamine, respectively. (C) The concentration versus time profile (black) is plotted, and kinetic modeling (orange) was calculated from the parameters: [dopamine $_{\mathrm{p}}=2.4 \mathrm{nM}, V_{\max }=0.13 \mu \mathrm{M} / \mathrm{s}$, and $K_{\mathrm{m}}=0.45 \mu \mathrm{M}$, with $\mathrm{R}^{2}=0.91$. (D) Background-subtracted cyclic voltammogram confirms that dopamine is detected.

\subsubsection{Kinetics values compared to other studies}

The uptake values for Drosophila larvae were similar to those previously found in mammals. $V_{\max }$ depends on the density of transporter expression, which can vary in different brain regions and in different tissue preparations. The $V_{\max }$ reported here for serotonin was similar to that in the substantia nigra reticulata in rat brain slices, ${ }^{27,38}$ and the $V_{\max }$ of dopamine was similar to that in the nucleus accumbens and prelimbic cortex areas of mice brain slices. ${ }^{41}$ The $K_{\mathrm{m}}$ values for both monoamines are of the same order of magnitude as values reported in mammals using electrical stimulations. ${ }^{15,24,38}$ Our $K_{\mathrm{m}}$ for serotonin uptake is also similar to the value of $0.64 \pm 0.10 \mu \mathrm{M}$ obtained from studies 
expressing Drosophila serotonin transporter in transfected cells, ${ }^{40}$ but the $K_{\mathrm{m}}$ of Drosophila dopamine transporter in transfected cells $(4.8 \pm 0.4 \mu \mathrm{M})$ is an order of magnitude larger than the value we report here. ${ }^{39}$ The $K_{m}$ values may vary with preparations as well, so methods to measure it in intact tissue are valuable. Previous studies of electrically evoked serotonin release in mammals have reported that the magnitude of the [Serotonin $]_{p}$ was much smaller in vivo compared with values from slice preparations, likely due to tighter control mechanisms in vivo. ${ }^{27,30,42}$ The [Serotonin] in the fly larval VNC was similar to the in vivo value of [Serotonin] $]_{p}$ in mammals. ${ }^{42}$ However, the $[\text { dopamine }]_{p}$ in flies was much smaller than mammalian [dopamine $]_{p}$ values of both electrical stimulated release ${ }^{28}$ and optically stimulated release in anesthetized rat with similar stimulation parameters. ${ }^{15}$ The expression of ChR2 may vary in Drosophila, and with the different drivers used to express it, affecting how many action potentials are evoked.

The clearance kinetics of monoamine neurotransmitters in intact Drosophila tissue have been estimated previously with electrochemistry. With these methods, the clearance portion of individual curves from stimulated release or exogenously applied monoamine is fit with an exponential decay and the initial rate of clearance used to make a Michaelis-Menten plot. ${ }^{8,43}$ The uptake parameters were determined for serotonin after continuous optical stimulations; however, the evoked peak concentrations are not high enough to produce $V_{\max }$. Thus, a full Michaelis-Menten curve is difficult to acquire and the kinetic parameters of clearance might be underestimated. ${ }^{8}$ The $V_{\max }$ and $K_{\mathrm{m}}$ for serotonin in Drosophila larval VNC using this method were $0.17 \pm 0.04 \mu \mathrm{M} / \mathrm{s}$ and $0.35 \pm$ $0.08 \mu \mathrm{M},{ }^{8}$ respectively, both significantly smaller than the values reported here (unpaired t test, $\mathrm{p}<0.0001$ for $V_{\max }$ and $\mathrm{p}<0.05$ for $K_{\mathrm{m}}$ ). For dopamine, exogenously applied neurotransmitter in Drosophila larval VNC was employed to span a wider range of 
analyte concentrations. ${ }^{43} \mathrm{~A}$ diffusional distortion has been reported with exogenous application, which could lower the apparent rates of uptake and result in a high $K_{\mathrm{m}}$ value $^{44}$ and this was corrected for using fumin flies that are dopamine transporter knockouts. The $V_{\max }$ and $K_{\mathrm{m}}$ were $0.11 \pm 0.02 \mu \mathrm{M} / \mathrm{s}$ and $1.3 \pm 0.6 \mu \mathrm{M}$, respectively, in that study, which are not significantly different from the dopamine values reported here (unpaired t test, $p>0.05$ ). However, this method cannot provide any information about release. Pulsed stimulations are easy to perform, and modeling endogenous release provides an easy way to obtain kinetic values for uptake as well as the amount of neurotransmitter released per stimulation pulse.

\subsection{Conclusions}

Pulsed optical stimulations can be used in Drosophila to evoke neurotransmitter release, and the release is dependent on pulse width, pulse number, and stimulation frequency. With the same number of pulses, low-frequency stimulations have a distinct, steady-state response compared to high frequency patterns, which are peak shaped. Release evoked by pulsed stimulations is lower than that evoked by continuous stimulation because there is more time for uptake between stimuli. The main advantage of using pulsed stimulations is that the release and uptake kinetics of serotonin and dopamine can be estimated without the need to produce large concentrations to reach $V_{\max } . V_{\max }$ and $K_{\mathrm{m}}$ for serotonin and dopamine in Drosophila are similar to their values in mammals. Our results demonstrate that pulsed optical stimulations combined with FSCV detection can be used to measure the effect of firing patterns on release and uptake kinetics, strengthening the utility of Drosophila as a model system for studying mechanisms of neurotransmission during behavior and neurological disorders. 


\subsection{Methods}

\subsubsection{Chemicals}

Chemicals were purchased from Sigma-Aldrich (St. Louis, MO). Solutions were made with Milli-Q water (Millipore, Billerica, MA). Electrode calibrations and Drosophila dissections were conducted using a modified Schneider's buffer (15.2 mM MgSO 4,21 $\mathrm{mM} \mathrm{KCl}, 3.3 \mathrm{mM} \mathrm{KH}_{2} \mathrm{PO}_{4}, 36 \mathrm{mM} \mathrm{NaCl}, 5.8 \mathrm{mM} \mathrm{NaH}_{2} \mathrm{PO}_{4}, 5.4 \mathrm{mM} \mathrm{CaCl}_{2}, 11.1 \mathrm{mM}$ glucose, $5.3 \mathrm{mM}$ trehalose, $\mathrm{pH}=6.2)$.

\subsubsection{Electrochemical Measurements}

Cylindrical carbon-fiber microelectrodes were fabricated in house from T-650 carbon fibers (a gift of Cytec Engineering Materials, West Patterson, $\mathrm{NJ}$ ) as previously described. ${ }^{45}$ Fast-scan cyclic voltammetry data were collected using a ChemClamp potentiostat (Dagan, Minneapolis, MN, n=0.01 headstage), PCI 6711 and 6052 computer interface cards (National Instruments, Austin, TX) and a home built break-out box. Data collection was computer controlled by the TarHeel CV software program (gift of Mark Wightman, University of North Carolina). To detect serotonin, we applied a modified waveform, from $0.2 \mathrm{~V}$ to $1.0 \mathrm{~V}$, then to $-0.1 \mathrm{~V}$ and back to $0.2 \mathrm{~V}$ vs. a $\mathrm{Ag} / \mathrm{AgCl}$ reference electrode at a scan rate of $1000 \mathrm{~V} / \mathrm{s}$ with a repetition rate of $10 \mathrm{~Hz} .^{46}$ To detect dopamine, the electrode was scanned from -0.4 to $1.3 \mathrm{~V}$ and back at a scan rate of 400 $\mathrm{V} / \mathrm{s}$ at $10 \mathrm{~Hz}$. Electrodes were calibrated with $1 \mu \mathrm{M}$ serotonin or $1 \mu \mathrm{M}$ dopamine before and after use in situ.

\subsubsection{Preparation of Ventral Nerve Cords}

Fly stocks were made as described previously. ${ }^{8,18}$ Flies containing wild-type Channelrhodopsin-2 $2^{1,47}$ with the genotype UAS-ChR2 (a gift from Christian Schroll, Universitat Wurzburg) were crossed to flies expressing Tph-GAL4 (a gift from Jaeson 
Kim, Korea Advanced Institute of Science and Technology) to generate homozygous lines with a Tph-GAL4; UAS-ChR2 genotype. Flies containing UAS-H134R-ChR2 (Bloomington stock center) were crossed to flies expressing Th-GAL4 (a gift from Jay Hirsh, University of Virginia) to generate homozygous lines with a UAS-H134R-ChR2; Th-GAL4 genotype. The H134R ChR2 single mutant has been proved in previous studies to show increased photocurrent size and higher reliability at low-frequency spiking but a drop-off in spike reliability at frequencies above $40 \mathrm{~Hz} .{ }^{12,48}$ Homozygous, 3day-old larvae were shielded from light and fed all-trans retinal, mixed with Red Star yeast (Red Star, Milwaukee, WI), for 2-3 days prior to the dissection. The fly dissection and all the measurements were performed at room temperature. The central nervous system of a 5-day-old wandering third instar larva (L3W) was dissected out in modified Schneider's buffer, and the optic lobes were removed by a horizontal cut across the anterior thorax region to yield an isolated ventral nerve cord (VNC) ${ }^{8}$ The isolated VNC was adhered neuropil side down onto the bottom of a Petri dish with $3 \mathrm{~mL}$ of buffer. The VNC was visualized under a $40 \times$ water immersion objective of a microscope (Carl Zeiss Microscopy, LLC) and an electrode was implanted using a micromanipulator into the VNC four to six segments away from the cut edge. The electrode was allowed to equilibrate for at least 5 min before data collection. Thirty seconds of baseline data were collected before each stimulation. 


\subsubsection{Optical Stimulation}

The optical stimulation setup consisted of a $473 \mathrm{~nm}$ diode laser (IkeCool Corporation, Los Angeles, CA) with a built-in optical fiber of core diameter $200 \mu \mathrm{m}$ and $1.5 \mathrm{~m}$ in length. The optical fiber was coupled to the fluorescent microscope and the laser beam was focused on the sample via the microscope objective lens. The laser was modulated with the Transistor-Transistor Logic (TTL)-input control port on the laser power supply, which was connected to the breakout box. The TTL input of the laser was driven by electrical pulses controlled by the TarHeel program. The program controlled the frequency, pulse width, and pulse number of the TTL pulses.

The maximum output of the laser was $50 \mathrm{~mW}$. However, we measured the optical power at the specimen plane with a commercial power meter (Coherent Incorporation, Santa Clara, CA) and found an approximately $94 \%$ power loss through the microscope. The effect of laser illumination intensity on stimulated serotonin release was tested with 2 s continuous stimulation (Figure 3.8). The evoked serotonin release increased with increasing illumination power (from $0.1 \mathrm{~mW}$ to $2.5 \mathrm{~mW}, 1$-way ANOVA, $p<0.01)$. The minimum illumination power required to induce detectable serotonin release was $0.1 \mathrm{~mW}$ and the $2.5 \mathrm{~mW}$ power intensity was chosen for all the stimulations in this work because it evoked the highest serotonin release without producing any artifacts. ${ }^{16}$ As shown in a recent study with optical stimulation in rat brain slices, a stimulation artifact can be observed when a carbon-fiber microelectrode is not placed deep enough in the brain tissue and can be eliminated by inserting the electrode deeper in the tissue or by reducing the laser power. ${ }^{16}$ Similar stimulating artifacts are observed in flies when the optical fiber of the laser was projected directly on top of the fly VNC or when the electrode was simply sitting in buffer. Laser radiation activates the carbon surface $^{49-51}$; thus, the artifact signals were probably due to laser activation of the carbon- 
fiber surface and a change in the background current. No artifact was observed when the laser was coupled through the microscope, likely due to the low power.

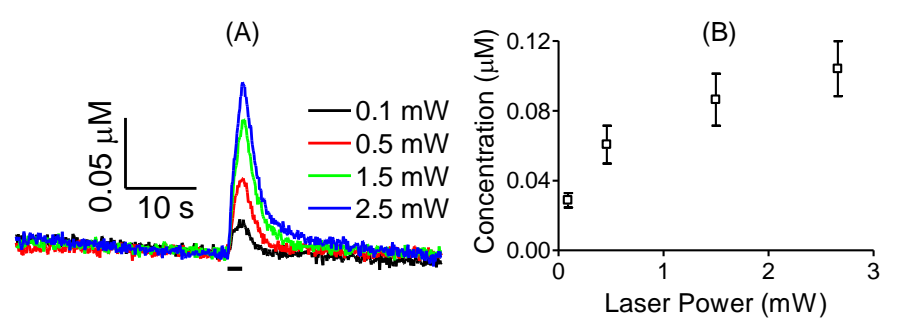

Figure 3.8 The effect of illumination power on stimulated serotonin release. $2 \mathrm{~s}$ continuous blue light was delivered via the light path of the fluorescent microscope through a $40 \times$ water immersion lens and the illumination power is tested to be from 0.1 $\mathrm{mW}$ to $2.5 \mathrm{~mW}$. (A) Representative serotonin responses with different illumination intensity values $(0.1,0.5,1.5,2.5 \mathrm{~mW}$ from bottom) in the same nerve cord. (B) Average effect of illumination intensity on serotonin release. Data are means $\pm S E M, n=7$.

\subsubsection{Statistics and Data Analysis}

Data are presented as mean \pm standard error of the mean (SEM) for $n$ number of fly samples and all error bars are given as SEM. One-way ANOVA with Bonferonni post-tests was used to compare effects among multiple groups. All statistics were performed in GraphPad Prism (GraphPad Software,Inc., La Jolla, CA). Curve fitting for release and uptake kinetics was performed using a nonlinear regression with a simplex minimization algorithm ${ }^{52,53}$ and the goodness of fit was described by the square of regression coefficient $\left(R^{2}\right)$. All $R^{2}$ values for curve fitting were greater than 0.83 . In most mammalian studies, $K_{\mathrm{m}}$ is fixed at an accepted value determined in brain synaptosomes or slices, and the program determines $V_{\max }$ and release per pulse. ${ }^{24,26,27,42}$ As there is no well published $K_{\mathrm{m}}$ value in fly synaptosomes, we chose to float all three parameters. An upper limit of $K_{\mathrm{m}}$ was set as a limitation of the iteration number, which was determined from the highest reporter literature values $(0.8 \mu \mathrm{M}$ for Drosophila serotonin transporter ${ }^{8,39,40}$ and $4.8 \mu \mathrm{M}$ for Drosophila dopamine transporter $\left.{ }^{39,43}\right)$. Thus, the program was stopped either when $K_{\mathrm{m}}$ reached the upper limit or a point where the iteration 
number kept increasing but the three parameters did not change. The average values of kinetic parameters were obtained from multiple stimulation curves with 4-ms pulse width at 4 different frequencies $(20 \mathrm{~Hz}, 40 \mathrm{~Hz}, 60 \mathrm{~Hz}$ and $80 \mathrm{~Hz})$. 


\subsection{Reference List}

(1) Nagel, G.; Szellas, T.; Huhn, W.; Kateriya, S.; Adeishvili, N.; Berthold, P.; Ollig, D.; Hegemann, P.; Bamberg, E. Proc. Natl. Acad. Sci. U. S. A. 2003, 100, 1394013945.

(2) Boyden, E. S.; Zhang, F.; Bamberg, E.; Nagel, G.; Deisseroth, K. Nat. Neurosci. 2005, 8, 1263-1268.

(3) Alilain, W. J.; Li, X.; Horn, K. P.; Dhingra, R.; Dick, T. E.; Herlitze, S.; Silver, J. J. Neurosci. 2008, 28, 11862-11870.

(4) Tsai, H. C.; Zhang, F.; Adamantidis, A.; Stuber, G. D.; Bonci, A.; de, L. L.; Deisseroth, K. Science 2009, 324, 1080-1084.

(5) Cardin, J. A.; Carlen, M.; Meletis, K.; Knoblich, U.; Zhang, F.; Deisseroth, K.; Tsai, L. H.; Moore, C. I. Nature 2009, 459, 663-667.

(6) Adamantidis, A. R.; Tsai, H. C.; Boutrel, B.; Zhang, F.; Stuber, G. D.; Budygin, E. A.; Tourino, C.; Bonci, A.; Deisseroth, K.; de, L. L. J. Neurosci. 2011, 31, 1082910835.

(7) Nichols, C. D. Pharmacology \& Therapeutics 2006, 112, 677-700.

(8) Borue, X.; Cooper, S.; Hirsh, J.; Condron, B.; Venton, B. J. J. Neurosci. Methods 2009, 179, 300-308.

(9) Vickrey, T. L.; Condron, B.; Venton, B. J. Anal. Chem. 2009, 81, 9306-9313.

(10) Ishizuka, T.; Kakuda, M.; Araki, R.; Yawo, H. Neurosci. Res. 2006, 54, 85-94.

(11) Lin, J. Y.; Lin, M. Z.; Steinbach, P.; Tsien, R. Y. Biophys. J. 2009, 96, 1803-1814.

(12) Nagel, G.; Brauner, M.; Liewald, J. F.; Adeishvili, N.; Bamberg, E.; Gottschalk, A. Curr. Biol. 2005, 15, 2279-2284.

(13) Gradinaru, V.; Thompson, K. R.; Zhang, F.; Mogri, M.; Kay, K.; Schneider, M. B.; Deisseroth, K. J. Neurosci. 2007, 27, 14231-14238.

(14) Wang, H.; Sugiyama, Y.; Hikima, T.; Sugano, E.; Tomita, H.; Takahashi, T.; Ishizuka, T.; Yawo, H. J. Biol. Chem. 2009, 284, 5685-5696.

(15) Bass, C. E.; Grinevich, V. P.; Vance, Z. B.; Sullivan, R. P.; Bonin, K. D.; Budygin, E. A. J. Neurochem. 2010, 114, 1344-1352.

(16) Bass, C. E.; Grinevich, V. P.; Kulikova, A. D.; Bonin, K. D.; Budygin, E. A. J. Neurosci. Methods 2013, 214, 149-155.

(17) Borue, X.; Condron, B.; Venton, B. J. J. Neurochem. 2010, 113, 188-199.

(18) Vickrey, T. L.; Venton, B. J. ACS Chem. Neurosci. 2011, 2, 723-729. 
(19) Paulk, A. C.; Zhou, Y.; Stratton, P.; Liu, L.; van, S. B. J. Neurophysiol. 2013, 110, 1703-1721.

(20) Murphy, D. L.; Lerner, A.; Rudnick, G.; Lesch, K. P. Mol. Interv. 2004, 4, 109-123.

(21) Lasky-Su, J. A.; Faraone, S. V.; Glatt, S. J.; Tsuang, M. T. Am. J. Med. Genet. B Neuropsychiatr. Genet. 2005, 133B, 110-115.

(22) Serretti, A.; Kato, M.; De, R. D.; Kinoshita, T. Mol. Psychiatry 2007, 12, 247-257.

(23) Brand, A. H.; Perrimon, N. Development 1993, 118, 401-415.

(24) John, C. E.; Budygin, E. A.; Mateo, Y.; Jones, S. R. J. Neurochem. 2006, 96, 267282.

(25) Threlfell, S.; Cragg, S. J.; Kallo, I.; Turi, G. F.; Coen, C. W.; Greenfield, S. A. J. Neurosci. 2004, 24, 8704-8710.

(26) Wightman, R. M.; Zimmerman, J. B. Brain Res. Brain Res. Rev. 1990, 15, 135-144.

(27) Bunin, M. A.; Prioleau, C.; Mailman, R. B.; Wightman, R. M. J. Neurochem. 1998, 70, 1077-1087.

(28) Garris, P. A.; Christensen, J. R.; Rebec, G. V.; Wightman, R. M. J. Neurochem. 1997, 68, 152-161.

(29) Jennings, K. A.; Lesch, K. P.; Sharp, T.; Cragg, S. J. J. Neurochem. 2010, 115, 965-973.

(30) Hashemi, P.; Dankoski, E. C.; Lama, R.; Wood, K. M.; Takmakov, P.; Wightman, R. M. Proc. Natl. Acad. Sci. U. S A 2012, 109, 11510-11515.

(31) Ernst, O. P.; Sanchez Murcia, P. A.; Daldrop, P.; Tsunoda, S. P.; Kateriya, S.; Hegemann, P. J. Biol. Chem. 2008, 283, 1637-1643.

(32) Bamann, C.; Kirsch, T.; Nagel, G.; Bamberg, E. J. Mol. Biol. 2008, 375, 686-694.

(33) Ritter, E.; Stehfest, K.; Berndt, A.; Hegemann, P.; Bartl, F. J. J. Biol. Chem. 2008, 283, 35033-35041.

(34) Foutz, T. J.; Arlow, R. L.; McIntyre, C. C. J. Neurophysiol. 2012, 107, 3235-3245.

(35) Mainen, Z. F.; Joerges, J.; Huguenard, J. R.; Sejnowski, T. J. Neuron 1995, 15, 1427-1439.

(36) Huguenard, J. R.; McCormick, D. A. J. Neurophysiol. 1992, 68, 1373-1383.

(37) Wu, Q.; Reith, M. E. A.; Wightman, R. M.; Kawagoe, K. T.; Garris, P. A. J. Neurosci. Methods 2001, 112, 119-133.

(38) Bunin, M. A.; Wightman, R. M. J. Neurosci. 1998, 18, 4854-4860. 
(39) Porzgen, P.; Park, S. K.; Hirsh, J.; Sonders, M. S.; Amara, S. G. Mol. Pharmacol. 2001, 59, 83-95.

(40) Corey, J. L.; Quick, M. W.; Davidson, N.; Lester, H. A.; Guastella, J. Proc. Natl. Acad. Sci. U. S A 1994, 91, 1188-1192.

(41) Mundorf, M. L.; Joseph, J. D.; Austin, C. M.; Caron, M. G.; Wightman, R. M. J. Neurochem. 2001, 79, 130-142.

(42) Hashemi, P.; Dankoski, E. C.; Wood, K. M.; Ambrose, R. E.; Wightman, R. M. J. Neurochem. 2011, 118, 749-759.

(43) Vickrey, T. L.; Xiao, N.; Venton, B. J. ACS Chem. Neurosci. 2013, 4, 832-837.

(44) Near, J. A.; Bigelow, J. C.; Wightman, R. M. J. Pharmacol. Exp. Ther. 1988, 245, 921-927.

(45) Swamy, B. E.; Venton, B. J. Analyst 2007, 132, 876-884.

(46) Jackson, B. P.; Dietz, S. M.; Wightman, R. M. Anal. Chem. 1995, 67, 1115-1120.

(47) Schroll, C.; Riemensperger, T.; Bucher, D.; Ehmer, J.; Voller, T.; Erbguth, K.; Gerber, B.; Hendel, T.; Nagel, G.; Buchner, E.; Fiala, A. Curr. Biol. 2006, 16, 1741-1747.

(48) Gunaydin, L. A.; Yizhar, O.; Berndt, A.; Sohal, V. S.; Deisseroth, K.; Hegemann, P. Nat. Neurosci. 2010, 13, 387-392.

(49) Poon, M.; Mccreery, R. L.; Engstrom, R. Anal. Chem. 1988, 60, 1725-1730.

(50) Strein, T. G.; Ximba, B. J.; Hamad, A. H. Electroanal. 1999, 11, 37-46.

(51) Farrell, P. C.; Kinley, P. R.; Weiss, D. J.; Strein, T. G. Electroanal. 2003, 15, 813820.

(52) Jones, S. R.; Garris, P. A.; Kilts, C. D.; Wightman, R. M. J. Neurochem. 1995, 64, 2581-2589.

(53) Garris, P. A.; Wightman, R. M. Regional differences in dopamine release, uptake and diffusion measured by fast-scan cyclic voltammetry. In Voltammetric Methods in Brain Systems, Boulton A.A., Baker G.B., Adams R.N., Eds.; Humana Press: Totowa, New Jersey., 1995; Vol. 27, pp 179-219. 


\section{Chapter 4}

\section{Characterization of dopamine releasable and reserve pools in Drosophila larva using} ATP/ P2X 2 mediated stimulation 


\section{Chapter 4: Characterization of dopamine releasable and reserve pools in Drosophila larva using ATP/ $\mathrm{P}_{2} \mathrm{X}_{2}$ mediated stimulation}

\section{Abstract}

The dopaminergic signaling pathways are conserved between mammals and Drosophila, but the factors important for maintaining the functional pool and the time course of regulation for dopamine are not fully understood in Drosophila. In this study, we characterized the releasable and reserve dopamine pools in Drosophila larva using $\mathrm{ATP} / \mathrm{P} 2 \mathrm{X}_{2}$ mediated stimulation. During repeated stimulations, dopamine release was stable with stimulations performed at least every $5 \mathrm{~min}$, but decayed with stimulations performed 2 min apart or less, indicating the replenishment of the releasable pool occurred on a time scale between 2 and 5 min. Dopamine synthesis or reuptake was pharmacologically inhibited to evaluated their contributions to maintaining the releasable dopamine pool. We found that both synthesis and reuptake were needed to maintain the releasable dopamine pool, with synthesis playing a major part in long-term replenishment and reuptake being more important for short-term replenishment. The time scale of synthesis and reuptake on the regulation of dopamine release in Drosophila is analogous to mammals. Furthermore, there was no cocaine-activated reserve pool of dopamine in Drosophila, a difference between the fly and mammals. Our study shows that both synthesis and reuptake replenish the releasable pool, providing a better understanding of dopamine regulation in Drosophila. 


\subsection{Introduction}

Dopamine signaling plays a major role in a variety of brain functions, including emotion, reward, cognition, memory, learning, locomotion and motor control. ${ }^{1,2}$ In the central nervous system, dopamine is released by exocytosis upon an action potential and acts in the extracellular space as a neurotransmitter. The amount of dopamine available for exocytosis determines the functional pool. Two main sources contributing to the releasable pool are newly synthesized dopamine and dopamine that is recycled from the extracellular space through reuptake by the dopamine transporter ${ }^{3}$. Understanding dopamine regulation is essential for the treatment of many neurological and psychiatric diseases such as Parkinson disease, Huntington disease, schizophrenia, and drug addiction. Dopamine pools have been studied extensively in mammalian models. For example, in mice, cocaine can increase dopamine release by activating a synapsindependent reserve pool of dopamine ${ }^{4,5}$.

Compared to rodent mammalian models, Drosophila melanogaster, the fruit fly, is an attractive model organism because of its simple nerve system, relatively short life cycle, and ease of molecular and genetic manipulation ${ }^{6}$. While genetically altered mice can take years to make, Drosophila genetic models can be produced in a few months. A variety of sophisticated genetic manipulations have been developed for Drosophila, allowing large-scale screening of mutants to model some aspect of human diseases. ${ }^{6,7}$ Our lab has developed methods for directly measuring dopamine in Drosophila and has verified that dopamine regulatory functions such as synthesis, uptake and vesicular release are conserved between Drosophila and mammals ${ }^{8,9}$. However, the factors important for maintaining the releasable dopamine pool in Drosophila are not fully understood.

Taking advantage of the fly genetics, several neural excitation methods with genetically encoded triggers have been successfully used in Drosophila ${ }^{10}$. Among these, 
ATP/P2X 2 mediated stimulation has become an elegant method for targeted control of neuronal activities. $\mathrm{P} 2 \mathrm{X}_{2}$ is a member of the ligand-gated cation channel $\mathrm{P} 2 \mathrm{X}$ family which is active by extracellular ATP. P2X $\mathrm{X}_{2}$ undergoes three sequential ATP binding steps in a cooperative manner ${ }^{11}$. Once fully bound, the channel opens rapidly and inward flow of cations leads to neuronal excitation ${ }^{12}$. A distinguishing characteristic of this channel is its slow desensitization, as currents at $\mathrm{P} 2 \mathrm{X}_{2}$ receptors decline little during sustained ATP application of a few seconds ${ }^{13,14}$. This feature makes it more suitable for inducing large concentration of neurotransmitter release compared to other cation channels. The Drosophila genome does not encode a homologous $\mathrm{P} 2 \mathrm{X}_{2}$ receptor homologous ${ }^{15}$ and previous studies suggest that there are no acute behavioral or physiologic effects of ATP in the absence of transgenic $\mathrm{P} 2 \mathrm{X}_{2}$ in Drosophila ${ }^{16,17}$. Thus, through genetic modification, $\mathrm{P}_{2} \mathrm{X}_{2}$ can be inserted into specific neurons and with exogenous applied ATP, those P2X $\mathrm{X}_{2}$-expressing neurons can be excited. ATP/P2 $\mathrm{X}_{2}$ mediated stimulation has been established in both larval and adult fly nervous systems to attain targeted neural excitation during behavioral and electrophysiology experiments ${ }^{17-19}$, but no ATP/P2X 2 mediated neurotransmitter release has been directly detected in Drosophila. Thus, for this methodology to be employed on a wider basis, it is essential to evaluate the parameters of $\mathrm{ATP} / \mathrm{P} 2 \mathrm{X}_{2}$ mediated stimulation on neurotransmitter release in Drosophila. In this study, we characterized ATP/P2X $\mathrm{X}_{2}$ mediated dopamine release and evaluated the roles of synthesis and reuptake in maintaining the releasable dopamine pool in Drosophila. Dopamine release was measured with fast-scan cyclic voltammetry (FSCV) at a carbon-fiber microelectrode implanted in the neruopil of an isolated larval fly ventral nerve cord (VNC), which was genetically modified to express $\mathrm{P}_{2} \mathrm{X}_{2}$ in dopaminergic cells. ATP was applied at various intervals with synthesis or reuptake pharmacologically inhibited to evaluate the recovery of the releasable pool. We found that both synthesis and reuptake are needed to maintain the releasable dopamine pool, 
with synthesis playing a major part in long-term replenishment and reuptake being more important for short-term replenishment. There was no cocaine-activated reserve pool of dopamine in Drosophila. Our study facilitates a better understanding of dopamine regulation in Drosophila, strengthening this model organism for the study of dopaminergic diseases.

\subsection{Results}

\subsubsection{Characterizing ATP/P2X 2 mediated dopamine release in Drosophila}

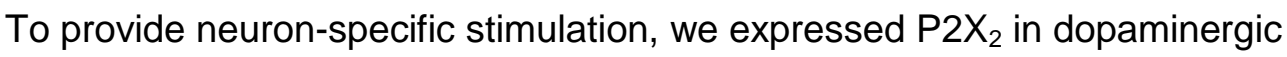
neurons using the yeast-based GAL4/ UAS system. Flies containing P2X $\mathrm{X}_{2}$, which is controlled by the upstream activating sequence (UAS) element, were crossed with flies containing GAL4 which is targeted to cells expressing tyrosine hydroxylase (th), a dopaminergic synthesis enzyme. Only one copy of the $\mathrm{P} 2 \mathrm{X}_{2}$ is needed and the flies work well as heterozygotes. A microelectrode was implanted into the neuropil and a capillary micropipette filled with ATP was inserted from the other side approximately 15-20 $\mu \mathrm{m}$ away from the electrode.

Picoliter volumes of ATP were pressure-ejected into the neuropil, and the dopamine response was monitored with FSCV. When 2 pmol of ATP is injected into buffer (Figure 4.1 A), the color plot and cyclic voltammogram show the characteristics of ATP with a primary oxidation peak close to the switching potential on the back scan ${ }^{20}$. Figure 4.1B shows that injection of 2 pmol ATP into a th-GAL4; UAS-P2 $\mathrm{X}_{2}$ larval VNC elicits dopamine release. The large green and blue areas on the color plot correspond to the oxidation and reduction of dopamine which are also confirmed by the characteristic oxidation and reduction peaks of the background-subtracted cyclic voltammogram. The dopamine response is slightly delayed after ATP injection, primarily because ATP has to 
diffuse from the micropipette to the area of the microelectrode where stimulated dopamine can be detected. Close to switching potential, there is a small current fluctuation upon ATP injection, which is also seen in a larval VNC without P2X (Figure $_{2}$ 4.1 C), likely due to changes in the background current caused by pressure changes. In the larval VNC without $\mathrm{P} 2 \mathrm{X}_{2}$, no dopamine response is observed when ATP is injected. In the fly tissue (Figure 4.1 B and C), no characteristic ATP peak is detectable. The oxidation potential for ATP is right around $1.3 \mathrm{~V}$, which is the switching potential used for the dopamine waveform, and the electron transfer kinetics at the carbon-fiber microelectrode are slower in tissue. Thus, ATP oxidation peak cannot be easily detected with the $1.3 \mathrm{~V}$ switching potential, although we could modify the waveform in the future to scan to $1.45 \mathrm{~V}$ if we wanted to better measure the ATP.

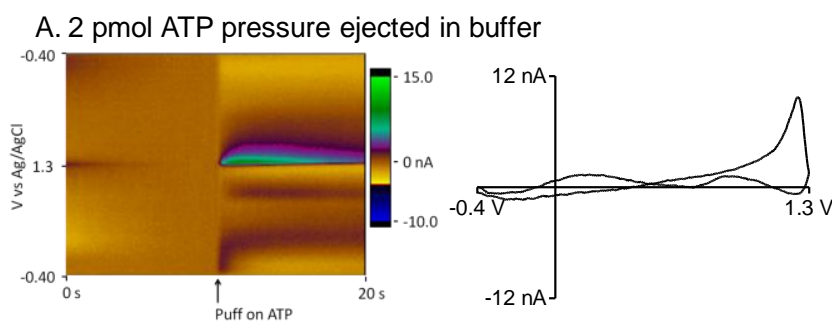

B. 2 pmol ATP pressure ejected in larval VNC expressing P2X2

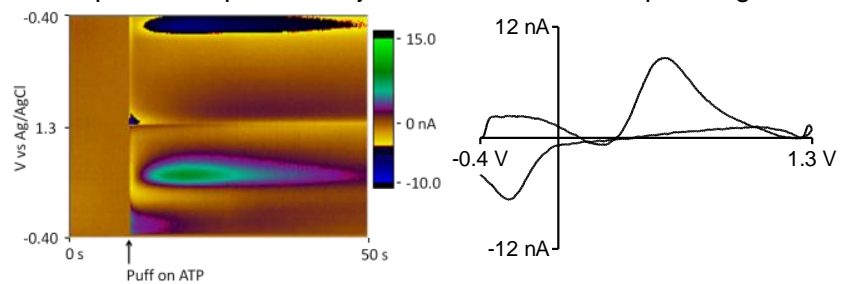

C. 2 pmol ATP pressure ejected in larval VNC without P2X2

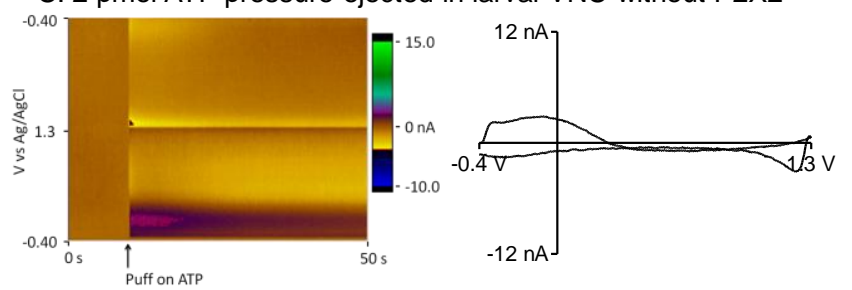

Figure 4.1 Characterization of ATP evoked dopamine signal in P2X $\mathrm{X}_{2}$ flies. Left column: false color plots with time on the x-axis, applied voltage on the y-axis and backgroundsubtracted faradaic current in pseudo-color. Injection of ATP is denoted by the arrow under the figure. Right column: background-subtracted cyclic voltammograms. (A) 2 pmol ATP was injected into buffer and the color plot and CV confirms it is ATP. (B) 2 
pmol ATP was injected into a larval VNC expressing $\mathrm{P}_{2} \mathrm{X}_{2}$ and the color plot and CV show that upon ATP stimulation, dopamine is released. (C) 2 pmol ATP was injected into a control larval VNC without $\mathrm{P}_{2} \mathrm{X}_{2}$ expression and the color plot shows minor fluctuations upon ATP injection corresponding to pressure error, but the CV does not show any characteristic dopamine peaks.

The peak dopamine concentration detected varies with the amount of ATP injected (Figure 4. 2). The data show a non-significant trend that the dopamine release increases as the amount of ATP increases until saturation is reached around 2 pmol ATP. At that level of ATP, approximately $0.4 \mu \mathrm{M}$ of dopamine is released. Thus, $2 \mathrm{pmol}$ of ATP was applied for the rest of the experiments.

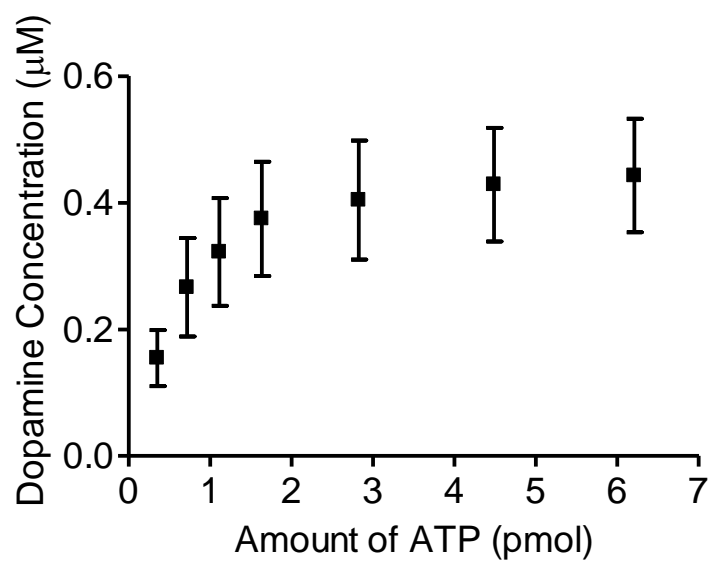

Figure 4.2 Evoked peak dopamine concentration varies with the amount of pressureinjected ATP. Data are presented as mean \pm SEM with $n=7$ samples. The pipette was filled with $200 \mu \mathrm{M}$ ATP and placed 15-20 $\mu \mathrm{m}$ away from the electrode. The data show a non-significant trend with the dopamine concentration increases with the amount of ATP applied until a plateau is reached around 2 pmol.

\subsubsection{Effect of stimulation intervals on dopamine release}

The stability of $\mathrm{ATP} / \mathrm{P} 2 \mathrm{X}_{2}$ induced dopamine release during multiple stimulations was tested (Figure 4. 3) by repeating stimulations at $0.5,1,2,5$ or 10 min intervals. For each animal, data were normalized to the peak concentration of the initial stimulation for easier comparison. Two-way ANOVA analysis shows a significant interaction of stimulation interval and stimulation number $(F 28,220]=5.291, \mathrm{p}<0.0001)$ and significant 
main effects of stimulation interval $(F 4,220]=236.7, p<0.0001)$ and stimulation number $(F 7,220]=14.98, p<0.0001)$. Dopamine release decayed when stimulations were performed at $0.5,1$ or 2 min interval but is relatively stable at 5 or 10 min interval, suggesting the replenishment of the releasable dopamine pool occurs between 2 and 5 min. For stimulations at $0.5,1$, or 2 min interval, dopamine release decreased sharply after the initial stimulation, and then decayed to a stable low level. On the second stimulation, the signal is $26 \%, 57 \%$ and $68 \%$ of the initial value for $0.5,1$ and $2 \mathrm{~min}$ interval, respectively. The dopamine concentration at this low level of the 0.5 min interval is significantly lower than that of the 1 and 2 min interval, while the difference between 1 and 2 min interval is not significant (Bonferroni post-test, see Table 4-1 for all statistics). We focused our analysis on the first five stimulations for pharmacological experiments.

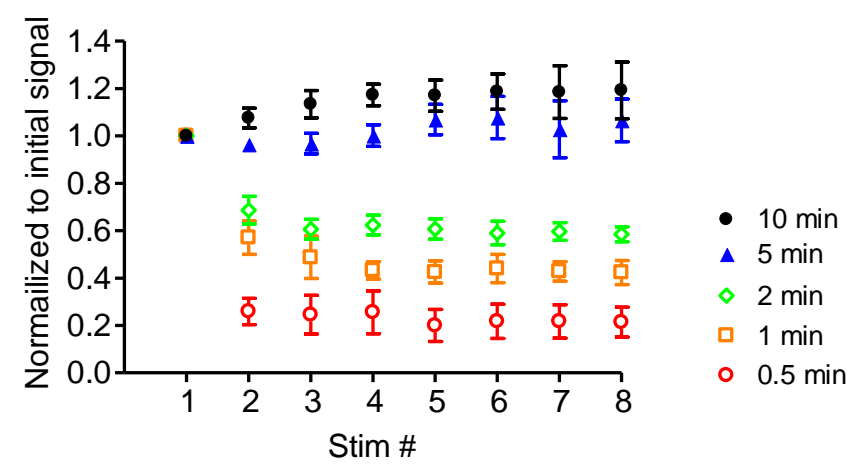

Figure 4.3 Dopamine release during stimulations repeated at $0.5,1,2,5$ or $10 \mathrm{~min}$ intervals. 2 pmol ATP was pressure injected and the pipette tip was kept 15-20 $\mu \mathrm{m}$ away from the electrode. Data are normalized to the peak concentration of the initial stimulation for each animal and are presented as mean $\pm S E M, n=6-7$. Dopamine release decays when stimulations are performed at $0.5,1$ and 2 min interval but is stable at 5 or 10 min interval. 


\begin{tabular}{|c|c|c|c|c|c|c|c|c|}
\hline \multicolumn{3}{|c|}{$30 \mathrm{~s}$ vs $1 \mathrm{~min}$} & \multicolumn{3}{|c|}{$1 \mathrm{~min}$ vs $2 \min$} & \multicolumn{3}{|c|}{$2 \min$ vs $10 \mathrm{~min}$} \\
\hline $\begin{array}{c}\text { Stim } \\
\text { Number }\end{array}$ & $\mathrm{t}$ & $P$ value & $\begin{array}{c}\text { Stim } \\
\text { Number }\end{array}$ & $t$ & $P$ value & $\begin{array}{c}\text { Stim } \\
\text { Number }\end{array}$ & $\mathrm{t}$ & $\mathrm{P}$ value \\
\hline Stim 1 & 0.000 & $P>0.05$ & Stim 1 & 0.000 & $P>0.05$ & Stim 1 & 0.000 & $P>0.05$ \\
\hline Stim 2 & 3.674 & $P<0.01$ & Stim 2 & 1.369 & $P>0.05$ & Stim 2 & 4.411 & $P<0.001$ \\
\hline Stim 3 & 2.85 & $P<0.05$ & Stim 3 & 1.400 & $P>0.05$ & Stim 3 & 5.986 & $P<0.001$ \\
\hline Stim 4 & 2.092 & $P>0.05$ & Stim 4 & 2.254 & $P>0.05$ & Stim 4 & 6.228 & $P<0.001$ \\
\hline Stim 5 & 2.664 & $P>0.05$ & Stim 5 & 2.059 & $P>0.05$ & Stim 5 & 6.152 & $P<0.001$ \\
\hline Stim 6 & 2.619 & $P>0.05$ & Stim 6 & 1.703 & $P>0.05$ & Stim 6 & 6.524 & $P<0.001$ \\
\hline Stim 7 & 2.492 & $P>0.05$ & Stim 7 & 1.910 & $P>0.05$ & Stim 7 & 6.431 & $P<0.001$ \\
\hline Stim 8 & 2.48 & $P>0.05$ & Stim 8 & 1.827 & $P>0.05$ & Stim 8 & 6.634 & $P<0.001$ \\
\hline \multicolumn{3}{|c|}{$30 \mathrm{~s}$ vs $2 \mathrm{~min}$} & \multicolumn{3}{|c|}{$1 \mathrm{~min}$ vs $5 \mathrm{~min}$} & \multicolumn{3}{|c|}{$5 \mathrm{~min}$ vs $10 \mathrm{~min}$} \\
\hline $\begin{array}{c}\text { Stim } \\
\text { Number }\end{array}$ & $\mathrm{t}$ & $P$ value & $\begin{array}{c}\text { Stim } \\
\text { Number }\end{array}$ & $\mathrm{t}$ & $P$ value & $\begin{array}{c}\text { Stim } \\
\text { Number }\end{array}$ & $\mathrm{t}$ & $\mathrm{P}$ value \\
\hline Stim 1 & 0.000 & $P>0.05$ & Stim 1 & 0.000 & $P>0.05$ & Stim 1 & 0.000 & $P>0.05$ \\
\hline Stim 2 & 5.042 & $P<0.001$ & Stim 2 & 4.464 & $P<0.001$ & Stim 2 & 1.216 & $P>0.05$ \\
\hline Stim 3 & 4.250 & $P<0.001$ & Stim 3 & 5.449 & $P<0.001$ & Stim 3 & 1.814 & $\mathrm{P}>0.05$ \\
\hline Stim 4 & 4.346 & $P<0.001$ & Stim 4 & 6.447 & $P<0.001$ & Stim 4 & 1.875 & $P>0.05$ \\
\hline Stim 5 & 4.619 & $P<0.001$ & Stim 5 & 7.300 & $P<0.001$ & Stim 5 & 1.101 & $P>0.05$ \\
\hline Stim 6 & 4.219 & $P<0.001$ & Stim 6 & 7.231 & $P<0.001$ & Stim 6 & 1.198 & $P>0.05$ \\
\hline Stim 7 & 4.304 & $P<0.001$ & Stim 7 & 6.803 & $P<0.001$ & Stim 7 & 1.716 & $P>0.05$ \\
\hline Stim 8 & 4.211 & $P<0.001$ & Stim 8 & 7.278 & $P<0.001$ & Stim 8 & 1.382 & $P>0.05$ \\
\hline \multicolumn{3}{|c|}{$30 \mathrm{~s}$ vs $5 \mathrm{~min}$} & \multicolumn{3}{|c|}{$1 \min$ vs $10 \min$} & & & \\
\hline $\begin{array}{c}\text { Stim } \\
\text { Number }\end{array}$ & $\mathrm{t}$ & $\mathrm{P}$ value & $\begin{array}{c}\text { Stim } \\
\text { Number }\end{array}$ & $\mathrm{t}$ & $\mathrm{P}$ value & & & \\
\hline Stim 1 & 0.000 & $P>0.05$ & Stim 1 & 0.000 & $P>0.05$ & & & \\
\hline Stim 2 & 7.993 & $P<0.001$ & Stim 2 & 5.726 & $P<0.001$ & & & \\
\hline Stim 3 & 8.188 & $P<0.001$ & Stim 3 & 7.332 & $P<0.001$ & & & \\
\hline Stim 4 & 8.457 & $P<0.001$ & Stim 4 & 8.393 & $P<0.001$ & & & \\
\hline Stim 5 & 9.860 & $P<0.001$ & Stim 5 & 8.443 & $P<0.001$ & & & \\
\hline Stim 6 & 9.747 & $P<0.001$ & Stim 6 & 8.474 & $P<0.001$ & & & \\
\hline Stim 7 & 9.197 & $P<0.001$ & Stim 7 & 8.584 & $P<0.001$ & & & \\
\hline Stim 8 & 9.661 & $P<0.001$ & Stim 8 & 8.712 & $P<0.001$ & & & \\
\hline \multicolumn{3}{|c|}{$30 \mathrm{~s}$ vs $10 \mathrm{~min}$} & \multicolumn{3}{|c|}{2 min vs 5 min } & & & \\
\hline $\begin{array}{c}\text { Stim } \\
\text { Number }\end{array}$ & $\mathrm{t}$ & $\mathrm{P}$ value & $\begin{array}{c}\text { Stim } \\
\text { Number }\end{array}$ & $\mathrm{t}$ & $\mathrm{P}$ value & & & \\
\hline Stim 1 & 0.000 & $P>0.05$ & Stim 1 & 0.000 & $P>0.05$ & & & \\
\hline Stim 2 & 9.256 & $P<0.001$ & Stim 2 & 3.149 & $P<0.05$ & & & \\
\hline Stim 3 & 10.07 & $P<0.001$ & Stim 3 & 4.104 & $P<0.001$ & & & \\
\hline Stim 4 & 10.40 & $P<0.001$ & Stim 4 & 4.281 & $P<0.001$ & & & \\
\hline Stim 5 & 11.00 & $P<0.001$ & Stim 5 & 5.051 & $P<0.001$ & & & \\
\hline Stim 6 & 10.99 & $P<0.001$ & Stim 6 & 5.326 & $P<0.001$ & & & \\
\hline Stim 7 & 10.98 & $P<0.001$ & Stim 7 & 4.715 & $P<0.001$ & & & \\
\hline Stim 8 & 11.10 & $P<0.001$ & Stim 8 & 5.252 & $P<0.001$ & & & \\
\hline
\end{tabular}

Table 4-1 Statistics for dopamine release during stimulations repeated at $0.5,1,2,5$ or 10 min intervals. Two way ANOVA shows a significant interaction of stimulation interval 
and stimulation number $(F[28,220]=5.291, \mathrm{p}<0.0001)$ and significant main effects of stimulation interval $(F 4,220]=236.7, \mathrm{p}<0.0001)$ and stimulation number $(F 7,220]=$ $14.98, p<0.0001)$. The table shows Bonferroni post test comparisons. Significant values are highlighted.

\subsubsection{Effect of synthesis inhibition and reuptake inhibition on stimulated release}

To evaluate the role and time course of synthesis and reuptake on replenishing the releasable dopamine pool, dopamine synthesis or reuptake was pharmacologically inhibited during repeated stimulations at 1 or 5 min intervals. Dopamine reuptake was inhibited with $60 \mu \mathrm{M}$ cocaine, a known dopamine transporter inhibitor ${ }^{21}$. Cocaine significantly prolongs evoked dopamine signaling in Drosophila larva ${ }^{8,9}$ and slows clearance of exogenous dopamine in Drosophila adults ${ }^{22}$. Dopamine synthesis was inhibited by $100 \mu \mathrm{M}$ 3-iodotyrosine. 3-iodotyrosine inhibits the activity of tyrosine hydroxylase, the rate-limiting enzyme in dopamine synthesis and significantly decreased steady-state amounts of dopamine after 2 days of feeding to Drosophila larvae ${ }^{23}$. In this study, we stimulated dopamine release in buffer first and then allowed 5 min for the dopamine pool to fully recover. After that we applied drug for $15 \mathrm{~min}$ and stimulated again. The dopamine signaling was compared before and after drug application. Our results showed after cocaine application, there was a significant increase in evoked dopamine concentration and the time to half decay $\left(t_{50}\right)$ (Paired test, $n=16, p<0.0001$ ), due to inhibition of dopamine reuptake. There is no significant change in dopamine concentration and $t_{50}$ value after 3 -iodotyrosine application (Paired t-test, $n=14, p=$ 0.2484 for concentration and $\mathrm{p}=0.2508$ for $t_{50}$ ).

Figure 4.4 shows the effects of cocaine and 3-iodotyrosine incubation on dopamine release during repeated stimulations performed at 1 and 5 min intervals. Twoway ANOVA shows a significant interaction of stimulation number and drug $(F 14,168]=$ 3.47, $\mathrm{p}<0.0001)$ and significant main effect of stimulation number $(F 7,168]=86.58$, 
$\mathrm{p}<0.0001)$ and drug $(F 2,168]=67.61, \mathrm{p}<0.0001)$ for the 1 min interval stimulation. The dopamine concentration decays significantly more in the cocaine group than in buffer (Bonferroni post-test, see Table 4-2 for all statistics). At the second stimulation, the dopamine release decreased to $15 \pm 3 \%$ of the initial value in cocaine compared to $57 \pm$ $7 \%$ in buffer. The decay in dopamine concentration for multiple stimulations in the 3iodotyrosine is not significantly different from that in buffer. For the $5 \mathrm{~min}$ interval stimulation, there is also a significant interaction of stimulation number and drug $(F 14,121]=11.77, \mathrm{p}<0.0001)$ and significant main effect of stimulation number $(F 7,121]$ $=24.26, \mathrm{p}<0.0001)$ and drug $(F 2,121]=405.17, \mathrm{p}<0.0001)$. While the release in buffer is stable, there is a significant decay both in the cocaine and 3-iodotyrosine, with the 3iodotyrosine group decaying significantly more (Bonferroni post-test, see Table 4-2 for all statistics). For the second stimulation, dopamine release in 3-iodotyrosine is $49 \pm 5 \%$ of the initial value compared to $68 \pm 3 \%$ in cocaine. For stimulations performed at 1 or 5 min interval, the decay is very similar with 3-iodotyrosine, while with cocaine, the decay at 1 min interval is significantly more than at 5 min interval (two way ANOVA with Bonferroni post-test, see Figure 4.5 and Table 4-3). Therefore, cocaine and 3iodotyrosine have different effects on dopamine release based on the interval during repeated stimulations. 
A. Stimulations 1 min apart

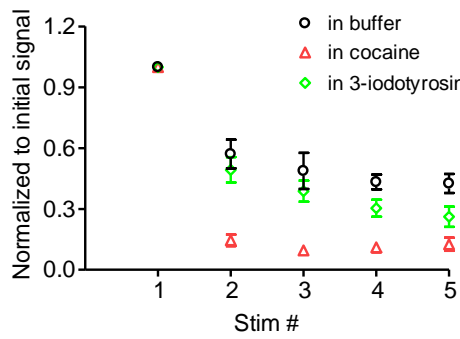

B. Stimulations 5 min apart
C. Concentration vs time at 1 min apart
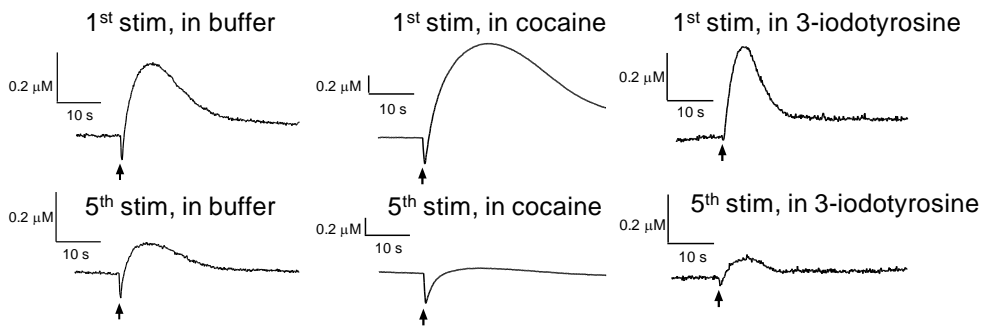

D. Concentration vs time at 5 min apart
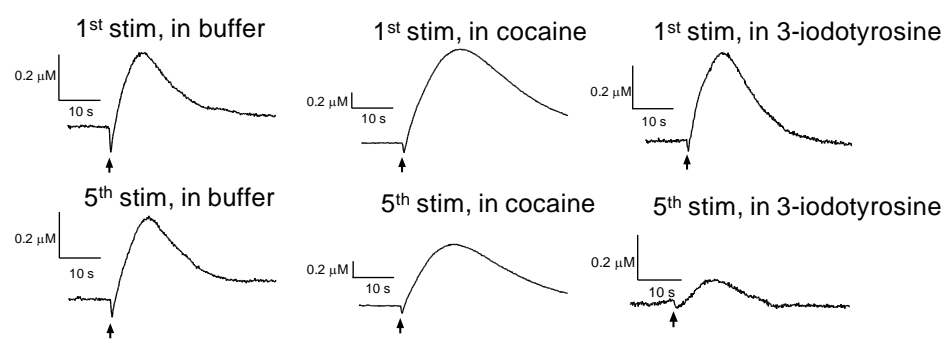

Figure 4.4 Effect of cocaine and 3-iodotyrosine on repeated stimulations. Stimulations were performed every $1 \mathrm{~min}(\mathrm{~A})$ or $5 \mathrm{~min}(\mathrm{~B})$. Dopamine reuptake was inhibited by 60 $\mu \mathrm{M}$ cocaine (triangles) or synthesis was inhibited by $100 \mu \mathrm{M} 3$-iodotyrosine (diamonds). Control samples in buffer are black circles. 2 pmol ATP was pressure injected and the pipette tip was kept 15-20 $\mu \mathrm{m}$ away from the electrode. Data are normalized to the peak concentration of the initial stimulation for each animal and are presented as mean \pm SEM, $n=5-7$. The representative concentrations vs time profiles are shown on the right for the first stimulation and the fifth stimulation for stimulations at $1 \mathrm{~min}$ interval $(\mathrm{C})$ or 5 min interval (D). The dopamine concentration is larger and the time response is slower in cocaine at the $1^{\text {st }}$ stimulation, while there is no difference in 3-iodotyrosine. Note the concentration scale for cocaine is different from the buffer and 3-iodotyrosine groups.

\begin{tabular}{|c|c|c|c|c|c|c|c|c|}
\hline \multicolumn{7}{|c|}{ Stimulations 1 min apart } \\
\hline \multicolumn{2}{|c|}{ buffer vs cocaine } & \multicolumn{2}{c|}{ buffer vs 3-iodotyrosine } & \multicolumn{2}{c|}{ cocaine vs 3-iodotyrosine } \\
\hline $\begin{array}{c}\text { Stim } \\
\text { Number }\end{array}$ & $\mathrm{t}$ & $\mathrm{P}$ value & $\begin{array}{c}\text { Stim } \\
\text { Number }\end{array}$ & $\mathrm{t}$ & $\mathrm{P}$ value & $\begin{array}{c}\text { Stim } \\
\text { Number }\end{array}$ & $\mathrm{t}$ & $\mathrm{P}$ value \\
\hline Stim 1 & 0.000 & $\mathrm{P}>0.05$ & Stim 1 & 0.000 & $\mathrm{P}>0.05$ & Stim 1 & 0.000 & $\mathrm{P}>0.05$ \\
\hline Stim 2 & 6.381 & $\mathrm{P}<0.001$ & Stim 2 & 1.137 & $\mathrm{P}>0.05$ & Stim 2 & 5.406 & $\mathrm{P}<0.001$ \\
\hline Stim 3 & 5.880 & $\mathrm{P}<0.001$ & Stim 3 & 1.462 & $\mathrm{P}>0.05$ & Stim 3 & 4.541 & $\mathrm{P}<0.001$ \\
\hline Stim 4 & 4.839 & $\mathrm{P}<0.001$ & Stim 4 & 1.889 & $\mathrm{P}>0.05$ & Stim 4 & 3.007 & $\mathrm{P}<0.05$ \\
\hline Stim 5 & 4.496 & $\mathrm{P}<0.001$ & Stim 5 & 2.398 & $\mathrm{P}>0.05$ & Stim 5 & 2.109 & $\mathrm{P}>0.05$ \\
\hline \multicolumn{7}{|c|}{ Stimulations 5 min apart } \\
\hline \multicolumn{7}{|c|}{ buffer vs cocaine } & buffer vs 3-iodotyrosine & cocaine vs 3-iodotyrosine \\
\hline Stim & $\mathrm{t}$ & $\mathrm{P}$ value & Stim & $\mathrm{t}$ & $\mathrm{P}$ value & Stim & $\mathrm{t}$ & $\mathrm{P}$ value \\
Number & \multicolumn{7}{|c|}{ Number } & \multicolumn{7}{c|}{ Number } & & \\
\hline Stim 1 & 0.000 & $\mathrm{P}>0.05$ & Stim 1 & 0.000 & $\mathrm{P}>0.05$ & Stim 1 & 0.000 & $\mathrm{P}>0.05$ \\
\hline Stim 2 & 4.230 & $\mathrm{P}<0.001$ & Stim 2 & 6.859 & $\mathrm{P}<0.001$ & Stim 2 & 2.888 & $\mathrm{P}<0.05$ \\
\hline Stim 3 & 3.705 & $\mathrm{P}<0.01$ & Stim 3 & 9.004 & $\mathrm{P}<0.001$ & Stim 3 & 5.299 & $\mathrm{P}<0.001$ \\
\hline Stim 4 & 4.586 & $\mathrm{P}<0.001$ & Stim 4 & 10.77 & $\mathrm{P}<0.001$ & Stim 4 & 6.186 & $\mathrm{P}<0.001$ \\
\hline Stim 5 & 5.967 & $\mathrm{P}<0.001$ & Stim 5 & 12.86 & $\mathrm{P}<0.001$ & Stim 5 & 6.897 & $\mathrm{P}<0.001$ \\
\hline
\end{tabular}


Table 4-2 Statistics for cocaine and 3-iodotyrosine on repeated stimulations. Two way ANOVA shows a significant interaction of stimulation number and drug $(F 14,168]=3.47$, $\mathrm{p}<0.0001)$ and significant main effect of stimulation number $(F 7,168]=86.58, \mathrm{p}<0.0001)$ and drug $(F 2,168]=67.61, \mathrm{p}<0.0001)$ for the 1 min interval stimulation. There are also a significant interaction of stimulation number and drug $(F[14,121]=11.77, \mathrm{p}<0.0001)$ and significant main effect of stimulation number $(F 7,121]=24.26, p<0.0001)$ and drug $(F 2,121]=405.17, \mathrm{p}<0.0001)$ for the 5 min interval stimulation. The table shows Bonferroni post test comparisons. Significant values are highlighted.

A. Cocaine

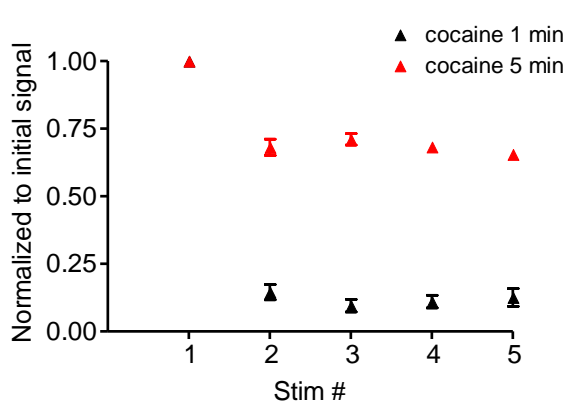

B. 3-iodotyrosine

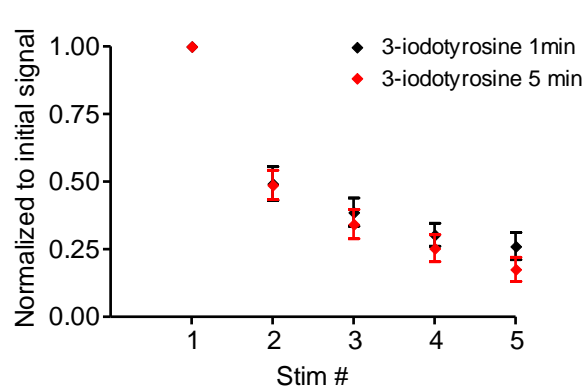

Figure $4.5(\mathrm{~A})$ In the presence of cocaine, dopamine release decays faster during repeated stimulations performed at 1 min interval than 5 min interval. (B) In the presence of 3-iodotyrosine, the decay is similar at $1 \mathrm{~min}$ and $5 \mathrm{~min}$ intervals.

\begin{tabular}{|l|c|l|l|c|c|}
\hline \multicolumn{2}{|c|}{ Cocaine 1 min vs 5 min } & \multicolumn{3}{c|}{ 3-iodotyrosine 1 min vs 5 min } \\
\hline Stim Number & $\mathrm{t}$ & $\mathrm{P}$ value & Stim Number & $\mathrm{t}$ & $\mathrm{P}$ value \\
\hline Stim 1 & 0.000 & $\mathrm{P}>0.05$ & Stim 1 & 0.000 & $\mathrm{P}>0.05$ \\
\hline Stim 2 & 9.673 & $\mathrm{P}<0.001$ & Stim 2 & 0.078 & $\mathrm{P}>0.05$ \\
\hline Stim 3 & 10.62 & $\mathrm{P}<0.001$ & Stim 3 & 0.731 & $\mathrm{P}>0.05$ \\
\hline Stim 4 & 9.885 & $\mathrm{P}<0.001$ & Stim 4 & 0.821 & $\mathrm{P}>0.05$ \\
\hline Stim 5 & 9.133 & $\mathrm{P}<0.001$ & Stim 5 & 1.399 & $\mathrm{P}>0.05$ \\
\hline
\end{tabular}

Table 4-3 Comparison of drug effect between $1 \mathrm{~min}$ and 5 min intervals. For cocaine, two way ANOVA shows a significant interaction of stimulation number and stimulation interval $(F 7,106]=12.01, \mathrm{p}<0.0001)$ and significant main effect of stimulation number $(F 7,106]=58.25, \mathrm{p}<0.0001)$ and stimulation interval $(F 1,106]=480.94, \mathrm{p}<0.0001)$. For 3 -iodotyrosine, there is no significant interaction of stimulation number and stimulation interval $(F[7,95]=0.48, \mathrm{p}=0.8491)$ and no significant main effect of stimulation interval $(F 1,95]=7.68, \mathrm{p}=0.0067)$ but a significant main effect of stimulation number $(F 7,95]=$ 405.17, $\mathrm{p}<0.0001)$. The table shows Bonferroni post test comparisons. Significant values are highlighted. 


\subsubsection{Investigating potential cocaine-activated reserve dopamine pool in Drosophila}

In mammals, cocaine increases the extracellular dopamine level by inhibiting reuptake but also by increasing dopamine release ${ }^{24,25}$. Cocaine can augment dopamine release after depletion of the readily releasable pool by activating release of a synapsinmediated vesicle reserve pool ${ }^{4,5}$. Here, we investigated if a cocaine-sensitive reserve pool existed in Drosophila. Dopamine synthesis was inhibited with $100 \mu \mathrm{M} 3-$

iodotyrosine and the releasable pool was depleted with consecutive stimulations at $1 \mathrm{~min}$ interval. Figure 4.6A shows after 8 simulations, the dopamine concentration decayed from $0.37 \pm 0.07 \mu \mathrm{M}$ to $0.05 \pm 0.01 \mu \mathrm{M}$. Then the sample was incubated with $60 \mu \mathrm{M}$ cocaine for $15 \mathrm{~min}$, and another four stimulations were performed. The dopamine signal increased to $0.19 \pm 0.02 \mu \mathrm{M}$ after cocaine application, and then progressively decayed to $0.04 \pm 0.01 \mu \mathrm{M}$ on the last stimulation. To confirm the effect of cocaine, a separate control group was conducted with the same experiments, except that the sample was incubated in buffer for 15 min after the depletion of releasable pool (Figure 4.6B). A similar trend was observed in the control group. The dopamine concentration decreased from $0.63 \pm 0.09 \mu \mathrm{M}$ to $0.05 \pm 0.02 \mu \mathrm{M}$ after 8 stimulations, then increased to $0.20 \pm 0.01$ $\mu \mathrm{M}$ after 15 min incubation in buffer, and progressively decayed to $0.03 \pm 0.01 \mu \mathrm{M}$ on the last stimulation. Thus, there is no significant difference in dopamine release between the cocaine or buffer group after the 15 min incubation (unpaired t test, $p=0.8306$ ). Our results indicate that cocaine does not significantly increase dopamine release after depletion of the readily releasable pool. However, there was a small increase of dopamine signal after 15 min incubation in either cocaine or buffer. It is likely that this dose of 3-iodotyrosine did not fully block synthesis, and thus the 15 min incubation time allowed dopamine to be partially re-synthesized. 
A. Cocaine group

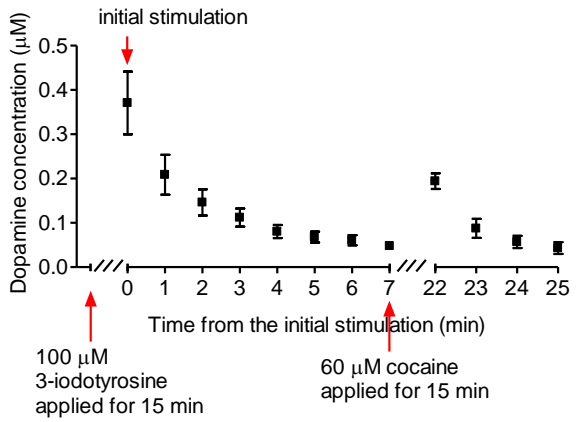

B. Buffer group

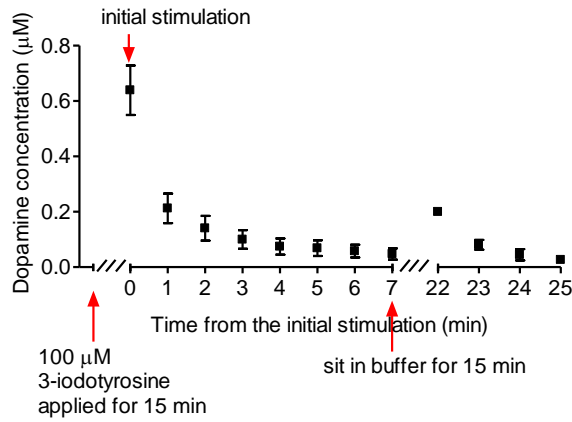

Figure 4.6 Effect of cocaine on dopamine release after repeated stimulations in the presence of a synthesis inhibitor. $100 \mu \mathrm{M}$ 3-iodotyrosine was applied for 15 min before the initial stimulation. (A) Eight consecutive stimulations were performed at $1 \mathrm{~min}$ intervals. At the end of the 8th stimulation, $60 \mu \mathrm{M}$ cocaine was applied for $15 \mathrm{~min}$, and another four stimulations were performed at 1 min interval, $n=8$. (B) $60 \mu \mathrm{M}$ of buffer was applied instead of cocaine, $n=5$. Data are presented as mean \pm SEM.

\subsection{Discussion}

While several groups have used electrophysiology studies to confirm the ability of ATP/P2X ${ }_{2}$ to stimulate targeted neurons ${ }^{18,19}$, this is the first time that ATP/P2X ${ }_{2}$ stimulated neurotransmitter release has been directly monitored in Drosophila. The amount of dopamine released increased with higher amounts of ATP applied, and then plateaued at $0.4 \mu \mathrm{M}$ with around $2 \mathrm{pmol}$ ATP, indicating that $\mathrm{P} 2 \mathrm{X}_{2}$ channels have been saturated or the releasable pool of dopamine was depleted. Previously, opticallystimulated dopamine release has been measured in flies which are genetically modified to express Channelrhodopsin-2 (ChR2), a blue-light activated cation channel in dopaminergic neurons ${ }^{8}$. Seven-second long stimulations resulted in $0.81 \pm 0.06 \mu \mathrm{M}$ of dopamine in those flies ${ }^{8}$, which is higher than that evoked by ATP/P2X $X_{2}$ in this study. This is likely because we used heterozygote flies with only one copy of the $\mathrm{P} 2 \mathrm{X}_{2}$ gene and thus the expression density of the $\mathrm{P} 2 \mathrm{X}_{2}$ channel is lower compared to the ChR2 flies, which are homozygous and have two copies of ChR2. Future studies with flies 
homozygous in $\mathrm{P}_{2} \mathrm{X}_{2}$ may have higher dopamine release, but the ability to stimulate release with only one copy of $\mathrm{P} 2 \mathrm{X}_{2}$ makes it useful for genetic studies in combination with other genetic mutants. Furthermore, the ATP/P2X $\mathrm{X}_{2}$ mediated release is suitable for measurements in brain regions where light penetration through the tissue is difficult. This might be useful in deeper neuropil in the adult fly, for example.

\subsubsection{The releasable dopamine pool is maintained by both synthesis and reuptake}

The time course of the recovery of the releasable dopamine pool was investigated by performing repeated stimulations at different intervals. Dopamine release was stable when stimulations were performed at least every $5 \mathrm{~min}$, while the signal decayed with stimulations performed 2 min apart, indicating the replenishment of the releasable dopamine pool occurred on a time scale between 2 and $5 \mathrm{~min}$. To investigate the contribution of synthesis and reuptake to the recovery of the releasable dopamine pool, pharmacological experiments were performed. During closely repeated stimulations ( $1 \mathrm{~min}$ apart), dopamine release decreased faster when reuptake was blocked, while the decay with synthesis inhibition was similar to that in buffer. Therefore, on the short time scale, reuptake is responsible for maintaining about $40 \%$ of the releasable pool. On the longer time scale, newly synthesized dopamine makes up about $50 \%$ and recycled dopamine about $30 \%$ of the releasable pool.

The control of dopamine release is similar in mammalian models. In mice striatal brain slices, the dopamine release elicited by a single stimulation pulse is steady with stimulation interval of 5 min but progressively decreases with stimulation interval of 3 min or less ${ }^{5}$. In the caudate nucleus of anesthetized rats, when long (10 s) stimulations are performed, a stimulation interval of approximately $20 \mathrm{~min}$ is required to achieve a reproducible response, but the dopamine release regains $80 \%$ by $5 \min ^{26}$. The $5 \mathrm{~min}$ time scale is consistent with our study in Drosophila. In anesthetized rats, the inhibition 
of synthesis affect stimulations performed 10 min apart, but not when they are less than 2 min apart, but the recycling of dopamine via uptake contributes to the short-term recovery of the releasable pool ${ }^{26,27}$. Thus, the timescale of synthesis and reuptake on maintaining the releasable dopamine pool in Drosophila is similar to that in mammals.

The role of synthesis and reuptake and their time courses on recovery of the releasable serotonin pool has also been investigated in Drosophila ${ }^{28}$. Reuptake is more important for the short time scale and synthesis on the longer time scale for serotonin as well as dopamine. However, in contrast to the fact that dopamine release recovery is independent of synthesis on the 1 min timescale, synthesis plays a role in the recovery of serotonin release at 1 min intervals ${ }^{28}$. This discrepancy suggests that synthesis functions on a different time scale for dopamine and serotonin signaling in Drosophila.

\subsubsection{There is no cocaine-activated reserve dopamine pool in Drosophila}

Study of electrically stimulated dopamine release in the striatum of anesthetized mice found that cocaine could elevate dopamine release by mobilization of a synapsindependent reserve pool of dopamine-containing vesicles ${ }^{4,5}$. Synapsins are phosphoproteins that bind to the cytosolic surface of synaptic vesicles and are important regulators of synaptic transmission. Biochemical studies suggest that the balance between the readily releasable and the reserve pool of synaptic vesicles is regulated by the phosphorylation status of synapsins with dephosphorylated synapsins caging synaptic vesicles and preventing the release of neurotransmitter, while synapsin phosphorylation initiates vesicle mobilization ${ }^{29-32}$. It is hypothesized that cocaine facilitates dopamine release by increasing presynaptic $\mathrm{Ca}^{2+}$ influx and thereby triggers release of the reserve pool as a result of $\mathrm{Ca}^{2+}$ dependent phosphorylation of synapsins ${ }^{4,5}$. Studies have identified two functionally and topographically distinct pools of synaptic vesicles in the boutons of the Drosophila larval neuromuscular junction ${ }^{33-36}$. Only one 
synapsin gene is found in the genome of Drosophila ${ }^{37}$, and evidence shows the synapsin mediates mobilization of the reserve pool during intense stimulations ${ }^{34}$. No reserve pool has been reported in the dopaminergic neurons in Drosophila.

We investigated the existence of the synapsin mediated reserve pool in dopaminergic neurons by depleting the readily releasable pool with inhibiting synthesis. Our results showed the dopamine release after cocaine application was not significantly different from that in the control group. Even though the possibility of a reserve dopamine pool cannot be ruled out, our study indicated there was no cocaine-sensitive reserve pool in the dopaminergic neurons in Drosophila larval VNC. This discrepancy between Drosophila and mammals should be taken into consideration when using Drosophila as a model organism for the study of cocaine addiction. Studies of serotonin regulation in Drosophila found that cocaine does not activate a large serotonin reserve pool, but immunohistochemistry indicates that not all serotonin content in the serotonergic neurons is available for release ${ }^{28}$. Thus, further study with immunohistochemistry staining nerve cords after depletion of the readily releasable pool could help to identify if reserve dopamine pool exists in Drosophila.

\subsection{Conclusions}

We have characterized ATP/P2X 2 mediated dopamine release in Drosophila larval ventral nerve cord and shown that ATP/P2X $\mathrm{X}_{2}$ mediated stimulation can be used as a substitute for light-activated channels. Two sources for releasable dopamine pool are identified, the newly synthesized dopamine and dopamine recycled via reuptake. These two sources act on a different time scale with reuptake responsible for rapid replenishment of the releasable pool and synthesis critical for longer term maintaining the releasable pool. The role and timescale of synthesis and reuptake on the regulation of dopamine signaling in Drosophila is analogous to mammals. Furthermore, even 
though two distinct pools of synaptic vesicles exist in Drosophila, there is no cocaine activated reserve pool in the dopaminergic neurons. Our study promotes a better understanding of dopamine regulation in Drosophila, thus facilitating the use of this model organism for the study of dopaminergic diseases.

\subsection{Methods}

\subsubsection{Chemicals}

All chemicals were purchased from Sigma-Aldrich (St. Louis, MO). Solutions were made with Milli Q water (Millipore, Billerica, MA). Electrode calibrations and Drosophila dissections were conducted in phosphate buffer $(131.25 \mathrm{mM} \mathrm{NaCl}, 3.0 \mathrm{mM}$ $\mathrm{KCl}, 10.0 \mathrm{mM} \mathrm{NaH}_{2} \mathrm{PO}_{4}, 1.2 \mathrm{mM} \mathrm{MgCl}_{2}, 2.0 \mathrm{mM} \mathrm{Na}_{2} \mathrm{SO}_{4}$, and $1.2 \mathrm{mM} \mathrm{CaCl}_{2}$ ) with 11.1 $\mathrm{mM}$ glucose, $5.3 \mathrm{mM}$ trehalose and $\mathrm{pH}$ adjusted to 7.4. ATP solutions filled in the picospritzing capillaries, ranging from 0.2 to $1 \mathrm{mM}$, were made with phosphate buffer. Stock solutions (1 $\mathrm{mM}$ ) of cocaine, fluxoetine and 3-iodotyrosine were made in water and the final concentration in the bath around the Drosophila VNC was $60 \mu \mathrm{M}$ cocaine, $60 \mu \mathrm{M}$ fluoxetine and $100 \mu \mathrm{M}$ 3-iodotyrosine.

\subsubsection{Preparation of Ventral Nerve Cords}

Flies containing UAS-P2X $\mathrm{X}_{2}$ on the third chromosome (a gift from Jayaraman Lab, Janelia Farm Research Campus) were crossed to files containing th-GAL4 on the second chromosome (a gift from J.Hirsh, University of Virginia) to generate a heterozygous line. The fly dissection and all the measurements were performed at room temperature. The central nervous system of a 5-day-old wandering third instar larva (L3W) was dissected out in phosphate buffer (with 11.1 mM glucose, $5.3 \mathrm{mM}$ trehalose).

The optic lobes were removed by a horizontal cut across the anterior thorax region to yield an isolated ventral nerve cord (VNC) and then an additional horizontal cut was 
made at the posterior-most portion of the ventral nerve cord to facilitate micropipette insertion. The isolated VNC was adhered neuropil side down onto the bottom of a Petri dish with $3 \mathrm{~mL}$ of buffer. The VNC was visualized under a $40 \times$ water immersion objective of a microscope (Carl Zeiss Microscopy, LLC), an electrode was implanted using a micromanipulator into the VNC four to six segments away from the cut edge and a picospritzing micropipette was inserted 15-20 $\mu \mathrm{m}$ away from the electrode. The electrode and micropipette were allowed to equilibrate after implantation for 10 min prior to data collection. Ten seconds of baseline data were collected before each stimulation.

\subsubsection{Electrochemical Measurements}

Cylindrical carbon-fiber microelectrodes were fabricated in house from T-650 carbon fibers (a gift of Cytec Engineering Materials, West Patterson, $\mathrm{NJ}$ ) as previously described ${ }^{38}$. Fast-scan cyclic voltammetry were performed using a ChemClamp potentiostat (Dagan, Minneapolis, MN, $\mathrm{n}=0.01$ headstage), $\mathrm{PCl} 6711$ and 6052 computer interface cards (National Instruments, Austin, TX) and a home-built breakout box. Data collection was computer controlled by the TarHeel CV software program (gift of Mark Wightman, University of North Carolina). The electrode was scanned from -0.4 to $1.3 \mathrm{~V}$ and back at a scan rate of $400 \mathrm{~V} / \mathrm{s}$ every $100 \mathrm{~ms}$ vs a $\mathrm{Ag} / \mathrm{AgCl}$ reference electrode. Electrodes were calibrated with $1 \mu \mathrm{M}$ dopamine before and after use in situ. For drug experiments, a second calibration was conducted in the presence of drug to account for possible drug effects on the electrode sensitivity.

Picospritzing micropipettes were made by pulling a $1.2 \mathrm{~mm} \times 0.68 \mathrm{~mm}$ glass capillary (A-M Systems, Carlsburg, WA) using a vertical pipette puller (Narishige, Japan). The tip of the micropipette was trimmed to make an opening. Micropipettes were filled with ATP solutions ranging from 0.2 to $1 \mathrm{mM}$, and ATP was pressure ejected with a Picospritzer III instrument (Parker Hannifin, Fairfield, NJ). The pipette was calibrated by 
ejecting ATP solution into oil and the diameter of the ejected droplet was measured to calculate the volume $\left(\right.$ volume $\left.=1 / 6 \pi d^{3}\right)$

\subsubsection{Statistics and Data Analysis}

All values are presented as mean \pm standard error of the mean (SEM) for $n$ number of fly samples and all error bars are given as SEM. All statistics were performed in GraphPad Prism (GraphPad Software,Inc., La Jolla, CA) and significance was considered at the $95 \%$ confidence level $(p<0.05)$. Paired t-test was performed to compare date before and after drugs in the same sample and unpaired t-test was used to compare date in two different groups. A one-way ANOVA with Bonferroni post-tests was performed to evaluate the effect of the amount of ATP on stimulated dopamine release. A two-way ANOVA with Bonferroni post-test was used to evaluate stimulation interval and drug effects during repeated stimulations. 


\subsection{Reference List}

(1) Schultz, W. Annu. Rev. Neurosci. 2007, 30, 259-288.

(2) Grace, A. A.; Floresco, S. B.; Goto, Y.; Lodge, D. J. Trends Neurosci. 2007, 30, 220-227.

(3) Venton, B. J.; Wightman, R. M. Anal. Chem. 2003, 75, 414A-421A.

(4) Venton, B. J.; Seipel, A. T.; Phillips, P. E.; Wetsel, W. C.; Gitler, D.; Greengard, P.; Augustine, G. J.; Wightman, R. M. J. Neurosci. 2006, 26, 3206-3209.

(5) Kile, B. M.; Guillot, T. S.; Venton, B. J.; Wetsel, W. C.; Augustine, G. J.; Wightman, R. M. J. Neurosci. 2010, 30, 9762-9770.

(6) Nichols, C. D. Pharmacol. Ther. 2006, 112, 677-700.

(7) Bier, E. Nat. Rev. Genet. 2005, 6, 9-23.

(8) Vickrey, T. L.; Condron, B.; Venton, B. J. Anal. Chem. 2009, 81, 9306-9313.

(9) Vickrey, T. L.; Xiao, N.; Venton, B. J. ACS Chem. Neurosci. 2013, 4, 832-837.

(10) Venken, K. J.; Simpson, J. H.; Bellen, H. J. Neuron 2011, 72, 202-230.

(11) Ding, S.; Sachs, F. J. Gen. Physiol 1999, 113, 695-720.

(12) North, R. A. Physiol Rev. 2002, 82, 1013-1067.

(13) Brake, A. J.; Wagenbach, M. J.; Julius, D. Nature 1994, 371, 519-523.

(14) Collo, G.; North, R. A.; Kawashima, E.; Merlo-Pich, E.; Neidhart, S.; Surprenant, A.; Buell, G. J. Neurosci. 1996, 16, 2495-2507.

(15) Littleton, J. T.; Ganetzky, B. Neuron 2000, 26, 35-43.

(16) Lima, S. Q.; Miesenbock, G. Cell 2005, 121, 141-152.

(17) Yao, Z.; Macara, A. M.; Lelito, K. R.; Minosyan, T. Y.; Shafer, O. T. J. Neurophysiol. 2012, 108, 684-696.

(18) Huang, J.; Zhang, W.; Qiao, W.; Hu, A.; Wang, Z. Neuron 2010, 67, 1021-1033.

(19) Hu, A.; Zhang, W.; Wang, Z. Proc. Natl. Acad. Sci. U. S A 2010, 107, 1026210267.

(20) Ross, A. E.; Venton, B. J. Analyst 2012, 137, 3045-3051.

(21) Greco, P. G.; Garris, P. A. Eur. J. Pharmacol. 2003, 479, 117-125. 
(22) Makos, M. A.; Kim, Y. C.; Han, K. A.; Heien, M. L.; Ewing, A. G. Anal. Chem. 2009, 81, 1848-1854.

(23) Neckameyer, W. S. Dev. Biol. 1996, 176, 209-219.

(24) Stamford, J. A.; Kruk, Z. L.; Millar, J. Neuropharmacology 1989, 28, 1383-1388.

(25) Lee, T. H.; Balu, R.; Davidson, C.; Ellinwood, E. H. Synapse 2001, 41, 301-310.

(26) Michael, A. C.; Ikeda, M.; Justice, J. B., Jr. Neurosci. Lett. 1987, 76, 81-86.

(27) Michael, A. C.; Ikeda, M.; Justice, J. B., Jr. Brain Res. 1987, 421, 325-335.

(28) Borue, X.; Condron, B.; Venton, B. J. J. Neurochem. 2010, 113, 188-199.

(29) Murthy, V. N. Nat. Neurosci. 2001, 4, 1155-1157.

(30) Jovanovic, J. N.; Sihra, T. S.; Nairn, A. C.; Hemmings, H. C., Jr.; Greengard, P.; Czernik, A. J. J. Neurosci. 2001, 21, 7944-7953.

(31) Cousin, M. A.; Malladi, C. S.; Tan, T. C.; Raymond, C. R.; Smillie, K. J.; Robinson, P. J. J. Biol. Chem. 2003, 278, 29065-29071.

(32) Chi, P.; Greengard, P.; Ryan, T. A. Neuron 2003, 38, 69-78.

(33) Kuromi, H.; Kidokoro, Y. Neuron 1998, 20, 917-925.

(34) Akbergenova, Y.; Bykhovskaia, M. Brain Res. 2007, 1178, 52-64.

(35) Kuromi, H.; Kidokoro, Y. Neuron 2002, 35, 333-343.

(36) Michels, B.; Diegelmann, S.; Tanimoto, H.; Schwenkert, I.; Buchner, E.; Gerber, B. Learn. Mem. 2005, 12, 224-231.

(37) Klagges, B. R.; Heimbeck, G.; Godenschwege, T. A.; Hofbauer, A.; Pflugfelder, G. O.; Reifegerste, R.; Reisch, D.; Schaupp, M.; Buchner, S.; Buchner, E. J. Neurosci. 1996, 16, 3154-3165.

(38) Swamy, B. E.; Venton, B. J. Analyst 2007, 132, 876-884. 
Chapter 5

Conclusions and Future Directions 


\section{Chapter 5: Conclusions and Future Directions}

This dissertation has focused on measurements of neurotransmitter signaling in Drosophila larval ventral nerve cord. I have described a method to modify microelectrodes with aligned carbon nanotube forests for enhanced electrochemical detection of neurotransmitters. Next, I investigated optically stimulated serotonin release with pulsed stimulation trains and studied the kinetics of serotonin release and clearance in Drosophila. Finally, a chemical stimulation method was exploited to characterize dopamine releasable and reserve pools in Drosophila. This final chapter summarizes the main conclusions and discusses future directions.

\subsection{Carbon nanotube-based microelectrodes for in vivo use}

In chapter II, I described a method to fabricate aligned CNTs on the surface of carbon-fiber microelectrodes using self-assembly techniques. The modified electrodes were highly sensitive and facilitated improved temporal resolution for the detection of neurotransmitters with FSCV. By aligning CNTs on the electrode surface, the ends of the CNTs which have similar properties to graphitic edge planes and are functionalized, ${ }^{1}$ were exposed to the analyte. This study supported the concept that the CNT ends provided the best electrochemistry for adsorption controlled molecules, such as dopamine. However, this modification increased the electrochemical complexity of the electrode. The fabrication went through multiple steps, true alignment of CNTs was difficult to achieve and the kinetics of CNTs were convoluted with the kinetics of carbon fibers. A logical next step is to develop electrodes made of pure, aligned CNTs.

Our group and another group have developed and characterized new CNT-yarn microelectrodes. ${ }^{2,3}$ By borrowing spinning techniques from the textile industry, CNTs grown using chemical vapor deposition techniques can be pulled and spun onto a rotating spool to make a yarn composed of nanotubes aligned and bundled together in a 
continuous macro-scale fashion. ${ }^{4,5}$ These CNT yarns are highly conductive, and are commercially available with controllable diameters ranging from 10 to $50 \mu \mathrm{m} .{ }^{6}$ Microelectrodes made of CNT yarns showed increased sensitive and selective, faster electron transfer kinetics and scan repetition frequency-independent dopamine current. ${ }^{2,3}$ Thus these CNT yarn electrodes are promising for in vivo use. Future studies could fabricate CNT yarn electrodes with a smaller size which can be implanted into the fly CNS. These electrodes would be useful for monitoring fast changes of small amount of neurotransmitter release in Drosophila. For example, the current modeling method for neurotransmitter release and clearance kinetics is based on the assumption that the release per pulse is constant during a pulsed stimulation train. ${ }^{7,8}$ This model is simplified, leaving out the regulation of autoreceptors. ${ }^{7,9}$ With the CNT yarn electrodes, the amount of neurotransmitter released by a single pulse during a pulsed stimulation train can be directly measured, thus helping to develop a more accurate kinetics modeling method. Another alternative way to make CNT-based microelectrodes is to directly grow vertically aligned CNTs on a metal micro-wire. The metal substrate should be electrochemical inactive for the detection of neurotransmitters, and thus only the characteristics of CNTs show. Compared to the CNT yarn electrodes, microelectrodes with CNT grown on micro-wire are more robust, thus facilitating penetration into the fly brain tissue, as each individual neuropil is surrounded by a glial sheath. ${ }^{10}$ Furthermore, measurements of neurotransmitter release in discrete regions of adult fly brain are challenging because each region may only be $20-50 \mu \mathrm{m}$ in diameter. The tip of the micro-wire can be controlled on the nanometer scale to be localized in discrete Drosophila brain regions without destroying them. 


\subsection{Future studies in Drosophila}

In chapter III and IV, I have described the use of two different ion channels, Channelrhodopsin-2 (ChR2) and P2X $\mathrm{X}_{2}$, to stimulate neurotransmitter release in Drosophila. Utilizing Drosophila's genetic palpability, we have genetically modified flies to express an ion channel in specific neural populations to evoke endogenous release of a specific neurotransmitter. These two ion channels have been proved to be robust to stimulate physiologically relevant neurotransmitter concentrations in Drosophila. Future study can explore the use of other ion channels to further improve the stimulation efficiency. For example, a new red light driven channelrhodopsin, Chrimson, has been identified recently. Chrimson is activated around $590 \mathrm{~nm}$, which is red shifted $100 \mathrm{~nm}$ more than ChR-2 and has been used in neurobehavioral studies in Drosophila to minimize visual system-mediated behavioral interference. ${ }^{11}$ Our lab is incorporating this new channelrhodopsin for targeted stimulation of neurotransmitter release. Compared to blue light stimulation, the red light has stronger cuticle penetration and thus allows for low level of light intensity to be used. Low level of red light would reduce tissue damage and stimulation artifact caused by blue light activation. ${ }^{12}$ Our preliminary work with this channel has shown it has higher stimulation efficiency than $\mathrm{ChR2}$ and robust neurotransmitter release can be detected in heterozygote flies with only one copy of the Chrimson gene. The ability to stimulate release with only one copy of Chrimson gene makes it useful for genetic studies in combination with other genetic mutants, thus facilitating optogenetic control of neurotransmitter release in a variety of fly mutants.

In chapter III, I reported a method to model release and clearance of endogenous serotonin, providing estimates of release and clearance kinetics in an intact CNS. The serotonin transporter (SERT) is of particular interest because polymorphisms in SERT in humans are linked to behavioral traits such as anxiety and depression ${ }^{13}$ and the selective serotonin reuptake inhibitors (SSRIs) are a class of anti-depressants. SSRIs 
are thought to improve mod in depressed patient by raising extracellular serotonin level. ${ }^{13}$ Studies have shown acute and chronic SSRI treatments affect serotonin synthesis in different ways. While acute SSRI application can suppress serotonin synthesis, ${ }^{14,15}$ chronic SERT inhibition and SERT knockout mutants in mammals has shown elevated synthesis, likely as an adaptation to decreased reuptake. ${ }^{16-18}$ The modeling method described in this dissertation allows the release and uptake kinetics to be estimated simultaneously and thus can be used to probe the basic mechanisms of tissue response to acute or chronic SERT inhibition. Using Drosophila as a model organism, these studies would be informative for future development of psychiatric treatments.

Another future study in the fly larvae is to probe the existence of serotonin autoreceptors. Although a serotonin autoreceptor has not yet been identified in Drosophila, studies have suggested serotonin release in the fly could be controlled by autoregulatory feedback. For example, the application of large amounts of exogenous serotonin elicits the retraction of serotonergic projections. ${ }^{19}$ Furthermore, pan-neural over-expression of drosophila $5-\mathrm{HT}_{1 \mathrm{~A}}$ receptor causes a significant decrease in the response to light of foraging 3rd instar larvae, suggesting that $5-\mathrm{HT}_{1 \mathrm{~A}}$ might act on serotonergic neurons as an autoreceptor thus modifying the larval response to light. ${ }^{20}$ The serotonin autoreceptor could be probed with our technique by monitoring stimulated release after administration of putative serotonin autoreceptor agonist and antagonist. This study will provide a better understanding of serotonin regulation in Drosophila. Measurements of neurotransmitter signaling in Drosophila larvae have proven the basic mechanisms underlying dopamine and serotonin regulation are analogous to mammals. The future direction in this research is to characterize dopamine and serotonin release in adult flies. Data from flies of different ages would be useful to understand dopaminerigic or serotonergic signaling across multiple stages of 
development and thus the effects of ageing on the monoaminergic systems can be explored. Mutants can be used to model the monoaminergic signaling changes across the life span in some in neurodegenerative diseases, such as Parkinson's and help to detect early abnormalities during these diseases. Furthermore, measurements in adult Drosophila could be directly correlated with behavioral and morphological changes, thus providing insight into the underlying neurological mechanisms.

\subsection{Final Remarks}

Overall, this dissertation describes methods to monitor changes in extracellular monoamine concentrations in Drosophila larvae. Targeted expression of either lightactivated or ligand-activated ion channels allowed specific depolarization of serotonergic or dopaminergic neurons. The stimulated neurotransmitter release was detected with sub-second resolution using FSCV at carbon-fiber microelectrodes. Aligned carbon nanotube modification of the electrode surface greatly improved sensitivity and selectivity without compromising temporal responses. With pulsed optical stimulation in ChR2-expressing flies, a modeling method which has been well established in mammalian models was exploited to estimate dopamine and serotonin release and clearance kinetics in Drosophila. We found the $V_{\max }$ and $K_{\mathrm{m}}$ for serotonin and dopamine in Drosophila are similar to their values in mammals, but the amount of neurotransmitter released per pulse is smaller. With $\mathrm{ATP} / \mathrm{P} 2 \mathrm{X}_{2}$ mediated stimulation, the releasable and reserve dopamine pools in Drosophila larva was characterized. We found both synthesis and reuptake were needed to maintain the releasable dopamine pool, with synthesis playing a major part in long-term replenishment and reuptake being more important for short-term replenishment and there was no cocaine-activated reserve pool of dopamine in Drosophila. These studies lead to a better understanding of dopamine and serotonin 
regulation in Drosophila, and strengthen the use of this model organism for the study of mechanisms underlying human behaviors and neurodegenerative diseases. 


\subsection{Reference List}

(1) Banks, C. E.; Compton, R. G. Analyst 2006, 131, 15-21.

(2) Jacobs, C. B.; Ivanov, I. N.; Nguyen, M. D.; Zestos, A. G.; Venton, B. J. Anal. Chem. 2014, 86, 5721-5727.

(3) Schmidt, A. C.; Wang, X.; Zhu, Y.; Sombers, L. A. ACS Nano 2013, 7, 7864-7873.

(4) Randeniya, L. K.; Bendavid, A.; Martin, P. J.; Tran, C. D. Small 2010, 6, 18061811.

(5) Zhao, H.; Zhang, Y.; Bradford, P. D.; Zhou, Q.; Jia, Q.; Yuan, F. G.; Zhu, Y. Nanotechnology 2010, 21, 305502.

(6) Jiang, C.; Li, L.; Hao, H. IEEE Trans. Neural Syst. Rehabil. Eng 2011, 19, 612-616.

(7) Bunin, M. A.; Prioleau, C.; Mailman, R. B.; Wightman, R. M. J. Neurochem. 1998, 70, 1077-1087.

(8) Garris, P. A.; Christensen, J. R.; Rebec, G. V.; Wightman, R. M. J. Neurochem. 1997, 68, 152-161.

(9) Wightman, R. M.; Zimmerman, J. B. Brain Res Rev. 1990, 15, 135-144.

(10) Stork, T.; Bernardos, R.; Freeman, M. R. Cold Spring Harb. Protoc. 2012, 2012, 117.

(11) Klapoetke, N. C.; Murata, Y.; Kim, S. S.; Pulver, S. R.; Birdsey-Benson, A.; Cho, Y. K.; Morimoto, T. K.; Chuong, A. S.; Carpenter, E. J.; Tian, Z.; Wang, J.; Xie, Y.; Yan, Z.; Zhang, Y.; Chow, B. Y.; Surek, B.; Melkonian, M.; Jayaraman, V.; Constantine-Paton, M.; Wong, G. K.; Boyden, E. S. Nat. Methods 2014, 11, 338346.

(12) Xiao, N.; Privman, E.; Venton, B. J. ACS Chem. Neurosci. 2014.

(13) Murphy, D. L.; Lerner, A.; Rudnick, G.; Lesch, K. P. Mol. Interv. 2004, 4, 109-123.

(14) Carlsson, A.; Lindqvist, M. J. Neural Transm. 1978, 43, 73-91.

(15) Stenfors, C.; Yu, H.; Ross, S. B. Naunyn Schmiedebergs Arch. Pharmacol. 2001, 363, 222-232.

(16) Stenfors, C.; Ross, S. B. Life Sci. 2002, 71, 2867-2880.

(17) Kim, D. K.; Tolliver, T. J.; Huang, S. J.; Martin, B. J.; Andrews, A. M.; Wichems, C.; Holmes, A.; Lesch, K. P.; Murphy, D. L. Neuropharmacology 2005, 49, 798-810.

(18) Homberg, J. R.; Olivier, J. D.; Smits, B. M.; Mul, J. D.; Mudde, J.; Verheul, M.; Nieuwenhuizen, O. F.; Cools, A. R.; Ronken, E.; Cremers, T.; Schoffelmeer, A. N.; Ellenbroek, B. A.; Cuppen, E. Neuroscience 2007, 146, 1662-1676. 
(19) Sykes, P. A.; Condron, B. G. Dev. Biol. 2005, 286, 207-216.

(20) Rodriguez Moncalvo, V. G.; Campos, A. R. BMC Neurosci. 2009, 10, 66. 